\title{
Finiteness of rigid cohomology with coefficients*
}

\author{
Kiran S. Kedlaya \\ Department of Mathematics, Room 2-165 \\ Massachusetts Institute of Technology \\ 77 Massachusetts Avenue \\ Cambridge, MA 02139 \\ kedlaya@math.mit.edu
}

November 2, 2005

\begin{abstract}
We prove that for any field $k$ of characteristic $p>0$, any separated scheme $X$ of finite type over $k$, and any overconvergent $F$-isocrystal $\mathcal{E}$ over $X$, the rigid cohomology $H_{\text {rig }}^{i}(X, \mathcal{E})$ and rigid cohomology with compact supports $H_{c, \text { rig }}^{i}(X, \mathcal{E})$ are finite dimensional vector spaces over an appropriate $p$-adic field. We also establish Poincaré duality and the Künneth formula with coefficients. The arguments use a pushforward construction in relative dimension 1, based on a relative version of Crew's conjecture on the quasi-unipotence of certain $p$-adic differential equations.
\end{abstract}

\section{Contents}

$\mathbf{1}$ Introduction $\quad 3$

1.1 Rigid cohomologv: an overview . . . . . . . . . . . . . . . . . . . . . . . . . .

1.2 The main results . . . . . . . . . . . . . . . . . . . . . . . . . . . . . . . . . . . .

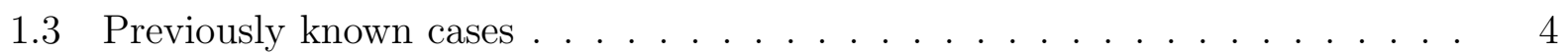

1.4 Approaches to $p$-adic cohomology . . . . . . . . . . . . . . . . 5

1.5 Structure of the papen . . . . . . . . . . . . . . . . . . . . . . . . . . . . . . . .

1.6 Further remarks . . . . . . . . . . . . . . . . . . . . . . 7

2 The rings of rigid cohomologv $\quad 8$

2.1 Affinoid algebras . . . . . . . . . . . . . . . . . . . . . . . . . . . . . . . . . . . .

2.2 Dagger algebras . . . . . . . . . . . . . . . . . . . . . . . . . . . . . . . . . . . . . . . . . . . . . . . . . .

2.3 Fringe algebras . . . . . . . . . . . . . . . . . . . . . . . . . 12

*2000 MSC number: 14F30. 
2.4 Interlude: a Gröbner basis calculation . . . . . . . . . . . . . . . . . . . 13

2.5 Robba rings . . . . . . . . . . . . . . . . . . . . . . . . . . 15

$\begin{array}{llr}3 & \text { Frobenius and differential structures } & 19\end{array}$

3.1 Differentials . . . . . . . . . . . . . . . . . . . . . . . . . 19

3.2 Algebras of MW-tvpe and Frobenius lifts . . . . . . . . . . . . . . . 20

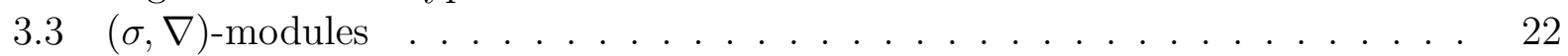

3.4 Cohomologv of $(\sigma . \nabla)$-modules . . . . . . . . . . . . . . . 23

\begin{tabular}{|lrl}
4 Rigid cohomology & 24
\end{tabular}

4.1 Some rigid geometrv . . . . . . . . . . . . . . . . . . . . . . . . 25

4.2 Overconvergent $F$-isocrvstals . . . . . . . . . . . . . . . . . . 25

4.3 Rigid cohomology with and without supports . . . . . . . . . . . 26

4.4 Additional properties . . . . . . . . . . . . . . . . . . . . . 28

\begin{tabular}{|lrl}
5 & Relative local monodromv & 29
\end{tabular}

5.1 Unipotence and $p$-adic local monodromv . . . . . . . . . . . . . . . . 29

5.2 More on unipotence . . . . . . . . . . . . . . . . . . . . . . . . . 30

5.3 Generic unipotence ... . . . . . . . . . . . . . . . . . . . . . . . . . . . . . . . 32

5.4 Generic quasi-unipotence . . . . . . . . . . . . . . . . . . . . . . . . . 34

\begin{tabular}{|lll}
\hline & Cohomology of curves & 36
\end{tabular}

6.1 Near-isomorphisms . . . . . . . . . . . . . . . . . . . . . . . . 36

6.2 Constant coefficients . . . . . . . . . . . . . . . . . . . . . . . 37

6.3 Interlude: matrix factorizations . . . . . . . . . . . . . . . . 40

6.4 General coefficients . . . . . . . . . . . . . . . . . . . . . . . . 41

\begin{tabular}{lll}
\hline & Pushforwards in rigid cohomology & 43
\end{tabular}

7.1 Relative local cohomologv . . . . . . . . . . . . . . . . . . . . 43

7.2 Base change in local cohomology . . . . . . . . . . . . . . . . . . 45

7.3 Generic pushforwards . . . . . . . . . . . . . . . . . . . . . . . . . 45

7.4 A Lerav spectral sequence . . . . . . . . . . . . . . . . . . . . . . . . 48

7.5 Pushforwards and base change . . . . . . . . . . . . . . . . . . . . . . 49

7.6 Pushforwards and external products . . . . . . . . . . . . . . . 50

8 Cohomology with compact supports $\quad 51$

8.1 The cohomologv of affine space . . . . . . . . . . . . . . . . . . . 51

8.2 The Poincaré pairing . . . . . . . . . . . . . . . . . . . . . 54

8.3 Review of $p$-adic functional analvsis . . . . . . . . . . . . . . 54

8.4 Topology of $(\sigma . \nabla)$-modules $\ldots \ldots \ldots \ldots \ldots \ldots$

8.5 Poincaré duality on affine space $\ldots \ldots \ldots \ldots$. . . . . . . . . . . . . 58

8.6 Relative Poincaré dualitv . . . . . . . . . . . . . . . . . . . . . . . . . . . . 59 
9 Finite dimensionalitv: the main results $\quad 60$

9.1 Cohomology without supports: the smooth case . . . . . . . . . . . . . 60

9.2 Cohomologv without supports: the general case . . . . . . . . . . . . . 62

9.3 Poincaré dualitv and cohomology with compact supports . . . . . . . . . . 63

9.4 The Künneth decomposition . . . . . . . . . . . . . . . . . . . . . 66

\section{Introduction}

\subsection{Rigid cohomology: an overview}

The purpose of this paper is to prove some basic structural properties about Berthelot's rigid cohomology for algebraic varieties over fields of characteristic $p>0$. This theory unifies most of the existing $p$-adic cohomology theories, including crystalline cohomology (developed by Berthelot, Ogus et al.; see [Be1] and [BO]), Monsky-Washnitzer cohomology (see [MW] and vdP), Serre's Witt vector cohomology (and its descendant, the de Rham-Witt complex of Deligne and Illusie; see [I] ), Lubkin's bounded Witt vector construction (see [Lu]), et al.

Rigid cohomology can be viewed as a version of algebraic de Rham cohomology in positive characteristic; in that theory, one defines cohomology of a variety by embedding it into a smooth variety and working on its formal neighborhood. To get a good theory (in which the divisions by $p$ inherent in integration make sense), Berthelot passes to rigid analytic spaces and replaces the formal neighborhoods by certain analytic neighborhoods (the "strict neighborhoods").

Grothendieck's proof that algebraic de Rham cohomology (of a variety over a field of characteristic zero) is finite dimensional uses a reduction to the case of a smooth proper variety via Hironaka's resolution of singularities. Similarly, using de Jong's alterations theorem to stand in for resolution of singularities, Berthelot [Be5], [Be6] succeeded in proving finite dimensionality of rigid cohomology with constant coefficients, along with Poincaré duality and the Künneth formula.

However, one can also work with nonconstant coefficients, analogous to local systems in de Rham cohomology; the most convenient coefficient objects are the "overconvergent $F$-isocrystals". In this paper, we generalize Berthelot's results to the cohomology of these coefficient objects.

\subsection{The main results}

Here are our results on the rigid cohomology of varieties over a field $k$ of characteristic $p>0$. Let $K$ be a complete discrete valuation ring of mixed characteristics $(0, p)$ with residue field $k$.

Theorem 1.2.1. Let $\mathcal{E} / K$ be an overconvergent $F$-isocrystal on a separated scheme $X$ of finite type over $k$. Then the rigid cohomology spaces $H_{\text {rig }}^{i}(X / K, \mathcal{E})$ are finite dimensional $K$-vector spaces for all $i$. 
Theorem 1.2.2. Let $\mathcal{E} / K$ be an overconvergent $F$-isocrystal on a separated scheme $X$ of finite type over $k$. Then the rigid cohomology spaces with compact supports $H_{c, \text { rig }}^{i}(X / K, \mathcal{E})$ are finite dimensional $K$-vector spaces for all $i$.

Theorem 1.2.3 (Poincaré duality). Let $\mathcal{E} / K$ be an overconvergent $F$-isocrystal on a smooth $k$-scheme $X$ of pure dimension $d$. Then for any closed subscheme $Z$ of $X$, there are natural perfect pairings

$$
H_{Z, \text { rig }}^{i}(X / K, \mathcal{E}) \otimes_{K} H_{c, \text { rig }}^{2 d-i}\left(Z / K, \mathcal{E}^{\vee}\right) \rightarrow K .
$$

Theorem 1.2.4 (Künneth formula). For $i=1,2$, let $\mathcal{E}_{i} / K$ be an overconvergent $F$ isocrystal on a separated scheme $X_{i}$ of finite type over $k$. Put $X=X_{1} \times_{k} X_{2}$, let $\mathrm{pr}_{i}$ : $X_{1} \times X_{2} \rightarrow X_{i}$ be the natural projections, and put $\mathcal{E}=\operatorname{pr}_{1}^{*} \mathcal{E}_{1} \otimes \operatorname{pr}_{2}^{*} \mathcal{E}_{2}$. Then there are natural isomorphisms

$$
\bigoplus_{j+l=i} H_{c, \text { rig }}^{j}\left(X_{1} / K, \mathcal{E}_{1}\right) \otimes_{K} H_{c, \text { rig }}^{l}\left(X_{2} / K, \mathcal{E}_{2}\right) \rightarrow H_{c, \text { rig }}^{i}(X / K, \mathcal{E}) .
$$

Moreover, if $X_{1}$ and $X_{2}$ are smooth over $k$, then for any closed subschemes $Z_{i}$ of $X_{i}$, there are also natural isomorphisms

$$
\bigoplus_{j+l=i} H_{Z_{1}, \text { rig }}^{j}\left(X_{1} / K, \mathcal{E}_{1}\right) \otimes_{K} H_{Z_{2}, \text { rig }}^{l}\left(X_{2} / K, \mathcal{E}_{2}\right) \rightarrow H_{Z_{1} \times_{k} Z_{2} / K, \text { rig }}^{i}(X, \mathcal{E}) .
$$

\subsection{Previously known cases}

Numerous special cases of these results have been established previously, modulo Berthelot's comparison theorems [Be5. Propositions 1.9 and 1.10] between rigid, crystalline and MonskyWashnitzer (MW) cohomology; we discuss the various strategies behind these methods in the next section. For now, we simply list the previously known special cases.

- For $\mathcal{E}$ trivial and $X$ smooth proper, all four results were proved in crystalline cohomology by Berthelot Be1]. Berthelot's result also applies to the case where $\mathcal{E}$ is obtained from an $F$-crystal of locally free coherent sheaves, via the comparison theorem between crystalline and rigid cohomologies with such coefficients [Q, Theorem 0.7.7].

- For $\mathcal{E}$ trivial and $X$ a smooth affine curve, Theorem 1.2.1 was proved in MW cohomology by Monsky [Mo].

- For $\mathcal{E}$ trivial and $X$ smooth affine, Theorem 1.2 .1 was proved in MW cohomology by Mebkhout [Me1], using results of Christol and Mebkhout [CM1].

- For $\mathcal{E}$ trivial and $X$ smooth, Theorems 1.2 .1 and 1.2 .2 were proved by Berthelot in [Be5, and Theorems 1.2 .3 and 1.2.4 in [Be6].

- For $\mathcal{E}$ trivial, Theorems 1.2 .1 and 1.2 .2 were proved by Grosse-Klönne GK2 and by Tsuzuki [T5]. 
- For $X$ a smooth affine curve, the first three results were proved conditionally by Crew $\mathrm{Cr}$ (see below).

- For $\mathcal{E}$ unit-root and $X$ smooth, all four results were proved by Tsuzuki [T3].

The finiteness result of Crew, for $X$ a curve but $\mathcal{E}$ arbitrary, relies on the hypothesis that $\mathcal{E}$ is quasi-unipotent at each point of a compactification of $X$. We will discuss this hypothesis further in the next section.

\subsection{Approaches to $p$-adic cohomology}

Before giving a description of what is actually in the paper, we take a moment to fit our methods into the already rich context of the existing literature on $p$-adic cohomology. This discussion focus on the past and present; for thoughts on the future, see Section 1.6.

One of the main strategies for approaching $p$-adic cohomology is to extract information from the case of smooth proper varieties. That is, one attempts to reduce consideration to crystalline cohomology. This is the strategy adopted by Berthelot, Tsuzuki, Shiho, et al.; the work of Grosse-Klönne is in a similar vein.

A second approach, practiced by Christol and Mebkhout, is perhaps the most direct heir to the legacy of Dwork and Robba. It is more analytic in nature, using strong structural properties of $p$-adic differential equations to control a suitable "Dworkian" cohomology theory; the most geometric of these is the "formal cohomology" introduced by Monsky and Washnitzer. It can be viewed as a close analogue of "naïve" algebraic de Rham cohomology of smooth affine schemes in characteristic zero.

A third approach, introduced by Crew, attempts to mediate the two extremes. It combines geometric arguments with arguments from $p$-adic functional analysis. As in the Dworkian approach, the aim is to prove assertions directly on affine schemes, then build general results from them, rather than have to deduce results on affines from results about proper schemes.

In all three points of view, the same obstruction arises to treating nonconstant coefficients: one needs an analogue for $p$-adic differential equations of basic monodromy properties of algebraic differential equations, or if one prefers, of Grothendieck's local monodromy theorem in étale cohomology. This obstruction was isolated by Crew, who posited the hypothesis that became known as "Crew's conjecture". (It is also sometimes called the Crew-Tsuzuki conjecture, since the "correct" formulation first appears in [T1.) Briefly put, Crew's conjecture states that every $p$-adic differential equation on an annulus with Frobenius structure has quasi-unipotent monodromy. (We give the precise statement in Chapter $\mathbf{5}$.)

Happily, this obstruction has recently been lifted. Crew's conjecture has been proved independently by André $\underline{\mathrm{An}}$, Mebkhout [Me2], and the author [Ke1]. (The unit-root case was proved earlier by Tsuzuki [T2] that case was a key ingredient to the aforementioned results [T3]. Incidentally, it also plays a pivotal role in [Ke1.) This circumstance makes it feasible to prove strong structural properties of rigid cohomology with coefficients; however, the "raw" form of Crew's conjecture is only sufficient for handling curves. One must refine 
the result into a more global form for it to be of cohomological value; one method of doing so was suggested by Shiho (see Section [1.6), but our method seems to be somewhat simpler. We formulate a version of Crew's conjecture for a differential equation on a family of annuli, and reduce it to the original result.

With a global version of Crew's conjecture in hand, we set off to obtain cohomological results. However, we do not particularly adhere to any of the approaches; our methods are something of a hodgepodge. For most of our calculations, we work with Monsky-Washnitzer cohomology; this is consonant with the approach of Mebkhout, although we tend to prefer algebraic and geometric constructions over $p$-adic differential equations. In particular, we construct "generic" higher direct images for families of affine curves, use a Leray spectral sequence to relate the cohomology of the total space to that of the direct images, then perform dévissage. The construction of the direct images, though algebraic in character, uses the formalism of Crew's functional analytic approach. However, to treat cohomology with compact supports and Poincaré duality, we pass to the functional analytic approach of Crew, and to manipulate cohomology on nonsmooth schemes, we invoke cohomological descent results of Chiarellotto and Tsuzuki. (It may also be possible to use Grosse-Klönne's methods to treat the nonsmooth case.)

\subsection{Structure of the paper}

We now give an overview of the content of the various sections of the paper.

In Chapter 2, we introduce the basic rings with which we compute: affinoid algebras (the building blocks of rigid analytic geometry), dagger algebras (the building blocks of MonskyWashnitzer cohomology and of Grösse-Klonne's theory of "dagger spaces"), the classical Robba ring, and our relative generalization thereof.

In Chapter 3, we construct auxiliary structures on the rings of Chapter 2 for use in rigid cohomology: modules of differential forms, and Frobenius lifts. These are used to define $(\sigma, \nabla)$-modules, the coefficient objects in the rigid cohomology of smooth affine schemes. (These may be thought of as "rigidified local systems": the differential forms provide the structure of a connection, and the Frobenius rigidifies the connection so as to make up for the disconnectedness of $p$-adic topologies, specifically by filling the role played by analytic continuation in complex analysis. On top of this, the Frobenius plays a role in controlling local monodromy analogous to that of a variation of Hodge structure on a punctured disc.)

In Chapter 4, we introduce Berthelot's rigid cohomology and its coefficient objects, the overconvergent $F$-isocrystals. These are closely related to the $(\sigma, \nabla)$-modules of the previous chapter, which are essentially "local models" of overconvergent $F$-isocrystals. We also recall (mostly without proof) some of the necessary formal properties of Berthelot's construction.

In Chapter 5, we state and prove the relative form of the $p$-adic local monodromy theorem. This states essentially that a family of $p$-adic differential equations on annuli, equipped with Frobenius structure, which by the original $p$-adic local monodromy theorem is quasiunipotent over the generic point, is also quasi-unipotent after restricting the base to an open dense subset. The proof is a reduction to the original form of Crew's conjecture, using a 
solution to a differential equation over the generic point as a guide for solving the relative equation.

In Chapter 6. we apply the (nonrelative) p-adic local monodromy theorem to prove, or rather to reprove, the finite dimensionality of rigid cohomology with coefficients on an affine curve. As mentioned above, assuming the $p$-adic local monodromy theorem, this has already been shown by Crew in $[\mathrm{Cr}$. However, our approach is very different: we use algebraic rather than topological consequences of the quasi-unipotence of differential equations. In any case, this chapter can be omitted at the preference of the reader.

In Chapter [7, we construct "relative Monsky-Washnitzer cohomology" for simple families of curves, namely products of a base with the affine line. (The basic strategy is enough to construct pushforwards more generally, at least in relative dimension 1, but this topic deserves a separate paper in itself.) We also show that the construction commutes with flat base change.

In Chapter 8, we compute the cohomology with compact supports of overconvergent $F$-isocrystals on the affine line, and establish Poincaré duality there. For the latter, we must bring in the topological machinery of $\mathrm{Cr}$.

Finally, in Chapter 9] we prove our main results using dévissage (i.e., induction on dimension), plus some other formal properties of rigid cohomology that do not occur elsewhere in the paper. As our methods are only adapted to smooth varieties, we must invoke cohomological descent results of Chiarellotto and Tsuzuki to handle nonsmooth varieties.

\subsection{Further remarks}

We conclude this introduction by pointing out some consequences, both actual and potential, of the present paper.

As an application of the finiteness of rigid cohomology with coefficients, one can now give a proof of the full Weil conjectures within the framework of $p$-adic cohomology, independent of the theory of étale cohomology. Moreover, one can give a $p$-adic analogue of "Weil II", the main theorem of Deligne's [De. We carry this out in a separate paper [Ke6]. (The reader is invited to imagine other results in étale cohomology that may have $p$-adic analogues; we will leave such speculation for another occasion.)

Our analogue of Weil II is not perfect, however, because our coefficient objects only mimic the category of lisse $\ell$-adic sheaves. The analogues of constructible sheaves should be holonomic arithmetic $\mathcal{D}$-modules (see [Be3] or [Be7]), but it remains to prove that they admit Grothendieck's six operations. This fault may eventually be circumvented by the notion of an "overholonomic" arithmetic $\mathcal{D}$, as introduced by Caro $\mathrm{Ca}$.

It may also be possible to establish results on $\mathcal{D}$-modules by proving a hypothesis of Shiho [Sh, Conjecture 3.1.8], which roughly states that every overconvergent $F$-isocrystal on a smooth $k$-scheme $X$ admits an extension to a log- $F$-isocrystal on some proper $k$-scheme containing an alteration of $X$ (in the sense of de Jong dJ1). Indeed, under this hypothesis, Shiho establishes the results of this paper by imitating the arguments of [Be5], Be6] with crystalline cohomology replaced by its logarithmic analogue. 
For isocrystals on a curve, Shiho's conjecture is de Jong's formulation of Crew's conjecture dJ2, which has been deduced from the usual formulation by the author Ke2]. In higher dimensions, it should be viewed as a globalized form of Crew's conjecture. Shiho's conjecture is known in the unit-root case by a result of Tsuzuki [T4; indeed, the results of [T3] rely on that case the same way that Shiho hopes to use the general assertion. See [Ke8] for the first paper in a projected series addressing Shiho's conjecture.

\section{Acknowledgments}

Part of this work was carried out during a visit to the Université de Rennes in May-June 2002; the author thanks Pierre Berthelot and Bas Edixhoven for their hospitality. The author also thanks Vladimir Berkovich, Johan de Jong and Arthur Ogus for helpful discussions, and the referees for numerous useful suggestions. The author was supported by an NSF Postdoctoral Fellowship and by NSF grant DMS-0400727.

\section{The rings of rigid cohomology}

In this section, we construct the rings that occur in our study of rigid cohomology. First, we adopt some notational conventions for the whole paper.

For the rest of the paper, $q$ will denote a fixed power of a prime $p$, and "Frobenius" will always mean the $q$-power Frobenius. Thus our notion of an $F$-isocrystal will coincide with what other authors call an $F^{a}$-isocrystal, where $a=\log _{p} q$.

Let $k$ be a field of characteristic $p>0$, and let $\mathcal{O}$ be a complete discrete valuation ring of characteristic 0 with residue field $k$ and fraction field $K$. Let $\Gamma^{*}$ be the divisible closure of the value group of $K$. Let $\pi$ be a uniformizer of $\mathcal{O}$. Let $v_{p}$ denote the valuation on $\mathcal{O}$ normalized so that $v_{p}(p)=1$, and let $|\cdot|$ denote the corresponding norm, that is, $|x|=p^{-v_{p}(x)}$. We will always assume extant and chosen a ring endomorphism $\sigma_{K}$ of $K$ preserving $\mathcal{O}$ and lifting the $q$-power Frobenius on $k$. For instance, if $k$ is perfect and $K=$ Frac $W(k), \sigma_{K}$ exists, is unique, and coincides with a power of the Witt vector Frobenius.

Recall that the direct (inductive) limit topology on the union $U$ of a direct system of topological spaces is the topology in which a subset of $U$ is open if and only if its preimage in each $U_{i}$ is open. In other words, it is the finest topology for which the maps $U_{i} \rightarrow U$ are continuous. If $U$ is Hausdorff, then any compact subset of $U$ is contained in some finite union of the $U_{i}$ (exercise, or see $\left[\mathrm{Gr}\right.$, Lemma 15.10]); in particular, a sequence $\left\{x_{n}\right\}$ converges to $x$ if and only if it does so within some finite union of the $U_{i}$.

\subsection{Affinoid algebras}

We recall the basic properties of affinoid algebras following [BGR, Chapter 6]. For an $n$-tuple $I=\left(i_{1}, \ldots, i_{n}\right)$ of nonnegative integers, write $x^{I}$ for $x_{1}^{i_{1}} \cdots x_{n}^{i_{n}}$ and $|I|=i_{1}+\cdots+i_{n}$. 
Definition 2.1.1. The Tate algebra (over $K$ ) in the variables $x_{1}, \ldots, x_{n}$ is defined as

$$
T_{n}=\left\{\sum_{I} a_{I} x^{I}: a_{I} \in K, \lim _{|I| \rightarrow \infty}\left|a_{I}\right|=0\right\} ;
$$

this ring is also denoted more explicitly $K\left\langle x_{1}, \ldots, x_{n}\right\rangle$. It is a noetherian ring BGR, Theorem 5.2.6/1]. The elements of $T_{n}$ are also referred to as strictly convergent power series in the $x_{i}$.

Definition 2.1.2. The Gauss norm on $T_{n}$ is given by the formula

$$
\left|\sum_{I} a_{I} x^{I}\right|=\max _{I}\left\{\left|a_{I}\right|\right\} .
$$

Under the Gauss norm, $T_{n}$ is complete, and every ideal of $T_{n}$ is closed BGR, Corollary $5.2 .7 / 2]$.

Definition 2.1.3. To any quotient $T_{n} / \mathfrak{a}$ of $T_{n}$ we can associate the residue norm:

$$
|f+\mathfrak{a}|=\inf \{|f+a|: a \in \mathfrak{a}\},
$$

which induces the quotient topology on $T_{n} / \mathfrak{a}$. Beware that this norm need not be powermultiplicative, e.g., if $T_{n} / \mathfrak{a}$ has nilpotent elements.

Definition 2.1.4. Any topological $K$-algebra isomorphic to a quotient of a Tate algebra is called an affinoid algebra (over $K$ ). It turns out that every $K$-algebra homomorphism between affinoid algebras is continuous BGR, Theorem 6.1.3/1], so the topology of an affinoid algebra is uniquely determined by the underlying $K$-algebra. We call that topology the intrinsic topology. Similarly, every finitely generated module over an affinoid algebra $A$ admits a unique topology, and all $A$-module homomorphisms between finitely generated modules are continuous [BGR, Proposition 3.7.3/3].

Definition 2.1.5. For $A$ an affinoid algebra, let Maxspec $A$ be the set of maximal ideals of $A$. For any $\mathfrak{m} \in$ Maxspec $A$, the field $A / \mathfrak{m}$ is a finite extension of $K$ [BGR, Corollary 6.1.2/3], so there is a unique norm $|\cdot|_{\mathfrak{m}}$ on $A / \mathfrak{m}$ extending the norm on $K$.

Definition 2.1.6. For $A$ an affinoid algebra, we say $A$ is reduced/normal/regular at a point $\mathfrak{m} \in \operatorname{Maxspec} A$ if the local ring of $A$ at $\mathfrak{m}$ is reduced/normal/regular. We may drop "at $\mathfrak{m}$ " if the property holds at all points, as in that case $A$ is reduced/normal/regular in the ring-theoretic sense [BGR, Corollary 7.3.2/9].

Definition 2.1.7. For $A$ an affinoid algebra, we define the spectral seminorm $|\cdot|_{\sup , A}$ on $A$ by

$$
|x|_{\sup , A}=\sup _{\mathfrak{m} \in \operatorname{Maxspec} A}|x|_{\mathfrak{m}}
$$


and the spectral valuation $v_{A}$ by

$$
v_{A}(x)=-\log _{p}\left(|x|_{\sup , A}\right) .
$$

We drop $A$ from the notation when it is clear from context. As catalogued in BGR, Section 6.2], the basic properties of the spectral seminorm include:

- The spectral seminorm is a $K$-algebra seminorm: that is, $|x+y|_{\text {sup }} \leq \max \left\{|x|_{\text {sup }},|y|_{\text {sup }}\right\}$, $|x y|_{\text {sup }} \leq|x|_{\text {sup }}|y|_{\text {sup }}$, and $|\lambda|_{\text {sup }}=|\lambda|$ for all $\lambda \in K$.

- The spectral seminorm is power-multiplicative: $|x|_{\text {sup }}^{n}=\left|x^{n}\right|_{\text {sup }}$ for $n \in \mathbb{N}$.

- Given any residue norm $|\cdot|$ on $A$, the spectral seminorm can be computed as

$$
|x|_{\text {sup }}=\lim _{n \rightarrow \infty}\left|x^{n}\right|^{1 / n}
$$

- The maximum modulus principle holds for $|\cdot|_{\text {sup }}$, that is, for any $x \in A$, there exists $\mathfrak{m} \in \operatorname{Maxspec} A$ such that $|x|_{\mathfrak{m}}=|x|_{\text {sup }}$. In particular, the values of $|\cdot|_{\text {sup }}$ all lie in $\Gamma^{*}$.

- If $f: A \rightarrow B$ is a $K$-algebra homomorphism between affinoid algebras, then $|f(a)|_{\text {sup }, B} \leq$ $|a|_{\text {sup }, A}$ for all $a \in A$.

- The spectral seminorm is a norm (that is, $|x|_{\text {sup }}=0$ if and only if $x=0$ ) if and only if $A$ is reduced. In this case we also refer to it as the spectral norm.

- The spectral norm on $T_{n}$ coincides with the Gauss norm.

Definition 2.1.8. For $A$ an affinoid algebra, we say that $x \in A$ is integral if $|x|_{\text {sup }, A} \leq 1$; the integral elements of $A$ form a subring of $A$, denoted $A^{\text {int }}$. We say that $x \in A$ is topologically nilpotent if $|x|_{\text {sup }, A}<1$, or equivalently (by [BGR. Proposition 6.2.3/2]) if $x^{n} \rightarrow 0$ as $n \rightarrow \infty$. The topologically nilpotent elements form an ideal of the integral subring; the quotient by this ideal is called the reduction of $A$. The reduction of $A$ is a finitely generated $k$-algebra BGR, Corollary 6.3.4/3]; its Spec is called the special fibre of $A$. Note that $A^{\text {int }} / \pi A^{\text {int }}$ is reduced if and only if it coincides with the reduction of $A$.

Definition 2.1.9. For $A$ an affinoid algebra and $f \in A$, the localization of $A$ at $f$ is a minimal affinoid algebra containing $A$ in which $f$ is invertible. The localization is unique up to canonical isomorphism; for $A=T_{n} / \mathfrak{a}$, it is given by

$$
A\left\langle f^{-1}\right\rangle=T_{n+1} /\left(\mathfrak{a} T_{n+1}+\left(x_{n+1} f-1\right)\right) .
$$

Definition 2.1.10. For $A_{1}$ and $A_{2}$ affinoid algebras, let $A_{1} \widehat{\otimes} A_{2}$ denote the completion of the usual tensor product. Then $A_{1} \widehat{\otimes} A_{2}$ is again an affinoid algebra, and it is in fact a product of $A_{1}$ and $A_{2}$ in the category of affinoid algebras and $K$-algebra morphisms. 


\subsection{Dagger algebras}

Next, we recall the basic properties of dagger algebras, as described in [GK1, Section 1].

Definition 2.2.1. For each $\rho>0$, define

$$
T_{n, \rho}=\left\{\sum_{I} a_{I} x^{I}: a_{I} \in K, \lim _{|I| \rightarrow \infty}\left|a_{I}\right| \rho^{|I|}=0\right\} ;
$$

then $T_{n, \rho}$ is an affinoid algebra for any $\rho \in \Gamma^{*}$ [BGR, Theorem 6.1.5/4]. Define the MonskyWashnitzer algebra (over $K$ ) in $x_{1}, \ldots, x_{n}$ as the union of the $T_{n, \rho}$ over all $\rho>1$; this ring is denoted $W_{n}$ or more explicitly $K\left\langle x_{1}, \ldots, x_{n}\right\rangle^{\dagger}$. The elements of $W_{n}$ are also referred to as overconvergent power series in the $x_{i}$.

By topologizing $W_{n}$ using the Gauss norm, we may view it as a dense subring of $T_{n}$. The ring $W_{n}$ is noetherian by a theorem of Fulton $\left[\mathrm{F}\right.$, and every ideal of $W_{n}$ is closed by the same proof as for $T_{n}$ (as pointed out in [GK1, Section 1.4]).

Definition 2.2.2. Define a dagger algebra to be any topological $K$-algebra isomorphic to a quotient of some $W_{n}$. Again, any $K$-algebra homomorphism between dagger algebras is automatically continuous [GK1, Proposition 1.6], so the topology is uniquely determined by the underlying $K$-algebra. We call this topology the affinoid topology, in order to distinguish it from a second topology on dagger algebras to be introduced in the next section.

All of the definitions of the previous section can be carried over to the category of dagger algebras. For instance, the localization of a dagger algebra $A=W_{n} / \mathfrak{a}$ at an element $f$ is given by

$$
A\left\langle f^{-1}\right\rangle^{\dagger}=W_{n+1} /\left(\mathfrak{a} W_{n+1}+\left(x_{n+1} f-1\right)\right) .
$$

For $A$ a dagger algebra, the completion $\widehat{A}$ of $A$ with respect to the ideal of topologically nilpotent elements is an affinoid algebra: if $f: W_{n} \rightarrow A$ is a surjection with kernel $\mathfrak{a}$, then the completion of $A$ is isomorphic to $T_{n} / \mathfrak{a} T_{n}$. Write $A^{\text {int }}=A \cap(\widehat{A})^{\text {int }}$. From [GK1, Theorem 1.7], we have the following relationships between a dagger algebra $A$ and its completion $\widehat{A}$.

- $\widehat{A}$ is faithfully flat over $A$.

- $\operatorname{Maxspec} A=\operatorname{Maxspec} \widehat{A}$.

- If $A$ is reduced (resp. normal, regular), so is $\widehat{A}$.

- If $A$ is reduced, its affinoid topology is induced by the spectral norm on $\widehat{A}$.

Thanks to the faithful flatness, every finitely generated $A$-module $M$ acquires a canonical "affinoid" topology, namely the subspace topology induced by the inclusion of $M$ into $M \otimes_{A}$ $\widehat{A}$. Again, all homomorphisms between finitely generated $A$-modules are continuous for this topology. 
Lemma 2.2.3. Let $A$ and $B$ be dagger algebras such that $A^{\text {int }} / \pi A^{\text {int }}$ and $B^{\text {int }} / \pi B^{\text {int }}$ are reduced. Let $f: A \rightarrow B$ be a K-algebra homomorphism of dagger algebras. If the induced map $R \rightarrow S$ on reductions is flat, then $f$ is flat, as is the induced map $A^{\mathrm{int}} \rightarrow B^{\mathrm{int}}$.

Proof. Since $A^{\text {int }} / \pi A^{\text {int }}$ and $B^{\text {int }} / \pi B^{\text {int }}$ are reduced, we have $R=A^{\text {int }} / \pi A^{\text {int }}$ and $S=$ $B^{\text {int }} / \pi B^{\text {int }}$, and $A^{\text {int }}$ and $B^{\text {int }}$ are both $\pi$-adically separated. Since the exact sequence $0 \rightarrow$ $\pi A^{\text {int }} \rightarrow A^{\text {int }} \rightarrow R \rightarrow 0$ remains exact upon tensoring with $B^{\text {int }}$ over $A^{\text {int }}, \operatorname{Tor}_{1}^{A^{\text {int }}}\left(R, B^{\text {int }}\right)$ vanishes. Thus [MW, Lemma 2.1] implies that the map $A^{\text {int }} \rightarrow B^{\text {int }}$ is flat, which implies in turn that $f$ is flat.

Remark 2.2.4. Although a dagger algebra $A$ is not complete with respect to the affinoid topology, it is "weakly complete" (in the terminology of [MW]) in the sense that for any $y_{1}, \ldots, y_{n} \in A^{\text {int }}$, the homomorphism $K\left[x_{1}, \ldots, x_{n}\right] \rightarrow A$ sending $x_{i}$ to $y_{i}$ extends to a continuous homomorphism $W_{n} \rightarrow A$ [GK1, 1.9]. For example, the series $(1-a)^{-1}=\sum_{n=0}^{\infty} a^{n}$ converges whenever $a$ is topologically nilpotent; hence any element of $A^{\text {int }}$ whose reduction is 1 is a unit.

\subsection{Fringe algebras}

Definition 2.3.1. For $A$ a dagger algebra, we define a fringe algebra to be any algebra $B$ of the form $f\left(T_{n, \rho}\right)$ for some surjection $f: W_{n} \rightarrow A$ and some $\rho \in \Gamma^{*}$ with $\rho>1$. (The name can be justified by visualizing Maxspec $B$ as being Maxspec $A$ plus some inessential "fringe" along its boundary.)

Because of the restriction on $\rho, B$ is an affinoid algebra, and so has an intrinsic topology. The intrinsic topology is finer than that induced from the affinoid topology of $A$; in particular, the latter is not complete.

The key technical tool for handling fringe algebras is the following lemma, for whose proof see [GK1, Lemma 1.8].

Lemma 2.3.2. Let $g: A_{1} \rightarrow A_{2}$ be a morphism of dagger algebras, and choose surjections $f_{i}: W_{n_{i}} \rightarrow A_{i}$. Then for any $\rho_{1}>1$, there exists $\rho_{2}>1$ such that $g\left(f_{1}\left(T_{n_{1}, \rho_{1}}\right)\right) \subseteq f_{2}\left(T_{n_{2}, \rho_{2}}\right)$.

Corollary 2.3.3. Let $g: A_{1} \rightarrow A_{2}$ be a $K$-algebra homomorphism between dagger algebras. Then any fringe algebra of $A_{1}$ maps under $g$ into a fringe algebra of $A_{2}$.

Corollary 2.3.4. For any fringe algebras $B_{1}, \ldots, B_{m}$ of $A$, there is a fringe algebra $B$ containing $B_{1}, \ldots, B_{m}$.

Proof. Choose a surjection $f: W_{n} \rightarrow A$. Then Lemma 2.3.2 implies that $B_{i} \subseteq f\left(T_{n, \rho}\right)$ for $\rho>1$ sufficiently small.

Corollary 2.3.5. Let $A$ be a dagger algebra and let $x \in A$ be a topologically nilpotent element for the affinoid topology. Then $x$ is topologically nilpotent in some fringe algebra $B$. 
Proof. Since $x$ is topologically nilpotent, we can find an integer $n$ and an element $\lambda$ of $K$ with $|\lambda|>1$ such that $x^{n} \lambda \in A^{\text {int }}$. By the weak completeness of dagger algebras, there is a $K$-algebra homomorphism from $W_{1}$ to $A$ taking $x_{1}$ to $x^{n} \lambda$. By Corollary 2.3.3, for any $\rho<|\lambda|$, the fringe algebra $T_{1}(\rho)$ maps into a fringe algebra $B$ of $A$; since $x_{1} \lambda$ is topologically nilpotent in $T_{1}(\rho)$, its image $x^{n}$ is topologically nilpotent in $B$, as then is $x$.

Remark 2.3.6. The converse of Corollary 2.3.5 does not hold. For instance, if $A=W_{n}$, then $x_{1}$ is topologically nilpotent in each fringe algebra of $A$ but not in the affinoid topology of $A$.

Definition 2.3.7. For $A$ a dagger algebra, define the fringe topology of $A$ to be the direct limit of the intrinsic topologies on its fringe algebras. (This topology is the one called the "direct limit topology" in GK1, 4.2].) Note that the fringe topology is Hausdorff, since it is finer than the affinoid topology. Also, by Corollary 2.3.3 any $K$-algebra homomorphism between dagger algebras is automatically continuous for the fringe topologies (as well as for the affinoid topologies). Moreover, any finitely generated $A$-module also acquires a fringe topology (by base extension from a suitable fringe algebra), and homomorphisms between finitely generated $A$-modules are automatically continuous for these fringe topologies (as well as for the affinoid topologies).

Definition 2.3.8. For $A_{1}, A_{2}$ dagger algebras, we define the completed tensor product $A_{1} \widehat{\otimes} A_{2}$ as the direct limit of $B_{1} \widehat{\otimes} B_{2}$ as each $B_{i}$ runs over all fringe algebras of $A_{i}$. Then $A_{1} \widehat{\otimes} A_{2}$ is again a dagger algebra, and (thanks to Lemma 2.3.2 and its corollaries) is a product of $A$ and $B$ in the category of dagger algebras and (continuous) $K$-algebra morphisms. For example,

$$
K\left\langle x_{1}, \ldots, x_{n}\right\rangle^{\dagger} \widehat{\otimes} K\left\langle y_{1}, \ldots, y_{n}\right\rangle^{\dagger} \cong K\left\langle x_{1}, \ldots, x_{n}, y_{1}, \ldots, y_{n}\right\rangle^{\dagger}
$$

For $A$ a dagger algebra, we write

$$
A\left\langle x_{1}, \ldots, x_{n}\right\rangle^{\dagger}=A \widehat{\otimes} K\left\langle x_{1}, \ldots, x_{n}\right\rangle^{\dagger} .
$$

Remark 2.3.9. If $A_{1}^{\text {int }} / \pi A_{1}^{\text {int }}$ and $A_{2}^{\text {int }} / \pi A_{2}^{\text {int }}$ are reduced, then $A_{1} \widehat{\otimes} A_{2}$ is flat over $A_{1}$ and $A_{2}$ by Lemma 2.2.3. Namely, if $R_{1}$ and $R_{2}$ are the corresponding reductions, then $R_{1}$ is vacuously flat over $k$ and so $R_{1} \otimes_{k} R_{2}$ is flat over $R_{2}$, and vice versa.

\subsection{Interlude: a Gröbner basis calculation}

The following lemma allows us to relate the spectral norm of a fringe algebra with that of its underlying dagger algebra. The proof is essentially a construction of a Gröbner basis; see E] for an introduction to this circle of ideas.

Lemma 2.4.1. Let $\mathfrak{a}$ be an ideal of $W_{n}$. Then there exists $\rho_{0}>1$ such that whenever $\rho \in\left(1, \rho_{0}\right] \cap \Gamma^{*}$, and $y \in W_{n}$ and $z \in T_{n, \rho}$ satisfy $y-z \in \mathfrak{a}$, we can find $u \in T_{n, \rho}$ with

$$
u-z \in \mathfrak{a}, \quad|u| \leq|y|, \quad|u|_{\rho} \leq|z|_{\rho},
$$

where $|\cdot|_{\rho}$ is the spectral norm on $T_{n, \rho}$. 
We note in passing that the argument also yields an alternate proof that $W_{n}$ and its integral subring are noetherian.

Proof. Define the partial ordering $\preceq$ on $\mathbb{Z}_{\geq 0}^{n}$ so that $I \preceq J$ when each component of $I$ is less than or equal to the corresponding component of $J$. Then $\preceq$ is a well partial ordering (wpo), i.e., any infinite sequence contains an nondecreasing subsequence. (To see this, first pass to a subsequence nondecreasing in the first component, then also in the second component and so on.)

Choose a total ordering $\leq$ of $\mathbb{Z}_{\geq 0}^{n}$ extending both $\preceq$ and the quasi-ordering by total degree, which is also compatible with addition (that is, $I \leq J$ if and only if $I+K \leq J+K$ ). For example, one may choose the "deglex" ordering, in which $I \leq J$ if either $I=J,|I|<|J|$, or $|I|=|J|$ and in the first position where $I$ and $J$ differ, $I$ has the lesser entry. Since $\preceq$ is a well partial ordering, $\leq$ is a well ordering. For $a=\sum a_{I} x^{I} \in T_{n, \rho}$, define the $\rho$ leading term of $a$ to be the expression $a_{I} x^{I}$, for $I$ the largest tuple under $\leq$ which maximizes $\left|a_{I} x^{I}\right|_{\rho}=\left|a_{I}\right| \rho^{|I|}$. (There is a largest such $I$ because there are only finitely many such $I$.)

We claim that for each $a \in W_{n}$, the 1-leading term of $a$ coincides with the $\rho$-leading term of $a$ for each sufficiently small $\rho>1$ (where the sense of "sufficiently small" depends on $a$ ). To see this, let $a_{I} x^{I}$ be the 1 -leading term of $a$. For each tuple $J=\left(j_{1}, \ldots, j_{n}\right)$, we then have either

(a) $\left|a_{J}\right|<\left|a_{I}\right|$, or

(b) $\left|a_{J}\right|=\left|a_{I}\right|$ and $J \leq I$; in this case we have $|J| \leq|I|$ since $\leq$ refines the quasi-ordering by total degree.

Pick $\eta>1$ for which $a \in T_{n, \eta}$. If $\left|a_{J} x^{J}\right|_{\eta} \leq\left|a_{I} x^{I}\right|_{\eta}$, then in case (a), we have $\left|a_{J} x^{J}\right|_{\rho}<\left|a_{I} x^{I}\right|_{\rho}$ for all $\rho \in[1, \eta) \cap \Gamma^{*}$; in case (b), we have $\left|a_{J} x^{J}\right|_{\rho} \leq\left|a_{I} x^{I}\right|_{\rho}$ and $J \leq I$. So these terms are all okay for any $\rho$; in fact, because $a \in T_{n, \eta}$, there are only finitely many tuples $J$ with $\left|a_{J} x^{J}\right|_{\eta}>\left|a_{I} x^{I}\right|_{\eta}$. For each such $J$, we must be in case (a), so $\left|y_{J} x^{J}\right|_{\rho}<\left|y_{I} x^{I}\right|_{\rho}$ for $\rho \in(1, \eta]$ sufficiently small. This yields the claim.

Define elements $a_{1}, a_{2}, \ldots$ of $\mathfrak{a}$ as follows. Given $a_{1}, \ldots, a_{i-1}$, choose $a_{i}$ if possible to be an element of $\mathfrak{a}$ whose 1-leading term is not a multiple of the 1-leading term of $a_{j}$ for any $j<i$, otherwise stop. Since $\preceq$ is a well partial ordering, this process must eventually stop; at that point, every 1-leading term of every element of $\mathfrak{a}$ occurs as a multiple of the 1-leading term for some $a_{i}$.

Let $A$ be the (finite) set of the $a_{i}$. By the argument above, we can choose $\rho_{0}>1$ such that for each $a \in A, a \in T_{n, \rho_{0}}$ and the $\rho_{0}$-leading term and 1-leading term of $a$ are equal. We claim that this choice of $\rho_{0}$ works. To see this, given $\rho \in\left(1, \rho_{0}\right] \cap \Gamma^{*}$, and $y \in W_{n}$ and $z \in T_{n, \rho}$ with $y-z \in \mathfrak{a}$ (and $y \neq 0$, or else $u=0$ trivially works), we construct a sequence $\left\{c_{j}\right\}$ of elements of $T_{n, \rho_{0}}$ and a sequence $\left\{d_{j}\right\}$ of elements of $A$, as follows. Given the sequences up to $c_{j}$ and $d_{j}$, put $z_{j}=z-c_{1} d_{1}-\cdots-c_{j} d_{j}$ (where if $j=0$ we take $z_{0}=z$ ). If $\left|z_{j}\right| \leq|y|$, then stop. Otherwise, let $e_{I} x^{I}$ be the leading term of $z_{j}-y$. By the construction of the $a_{i}$, we can find a monomial $c_{j+1}$ and an element $d_{j+1} \in A$ so that $c_{j+1} d_{j+1}$ has 1 -leading term, and hence $\rho$-leading term, equal to $e_{I} x^{I}$. 
From the construction, we clearly have $\left|z_{j}\right|_{\rho} \leq|z|_{\rho}$. On the other hand, if the process were never to terminate, we would have $\left|z_{j}\right| \rightarrow 0$ as $j \rightarrow \infty$ : if $\left|z_{j}\right|=\left|z_{j+1}\right|$, the 1-leading term of $z_{j+1}$ must be smaller under $\leq$ than that of $z_{j}$, and well-orderedness says this cannot continue forever, so eventually $\left|z_{j}\right|$ drops (by a discrete factor). Since $y \neq 0$, this yields a contradiction. Thus the process terminates at some $z_{j}$, and we may take $u=z_{j}$.

Our principal application of Lemma 2.4.1 is the following result, reminiscent of the Hadamard three circles theorem.

Proposition 2.4.2. Let $A$ be a dagger algebra, and let $B$ be a fringe algebra of $A$. Then for any rational $\epsilon \in(0,1]$, there exists a fringe algebra $C$ of $A$ containing $B$ such that

$$
|x|_{\mathrm{sup}, C} \leq|x|_{\sup , A}^{1-\epsilon}|x|_{\sup , B}^{\epsilon}
$$

for all $x \in B$.

Proof. Choose a surjection $f: W_{n} \rightarrow A$, and let $|\cdot|_{A}$ denote the induced residue norm on $A$. By Corollary 2.3.4, we can choose $\rho>1$ such that $f\left(T_{n, \rho}\right)$ contains $B$; by shrinking $\rho$ further, we can ensure that $\rho \leq \rho_{0}$ for some $\rho_{0}$ as in Lemma 2.4.1. Put $B^{\prime}=f\left(T_{n, \rho}\right)$, and let $|\cdot|_{B^{\prime}}$ denote the residue norm on $B^{\prime}$ induced by the spectral norm on $T_{n, \rho}$.

Let $|\cdot|_{\eta}$ denote the spectral norm on $T_{n, \eta}$. For $y \in T_{n, \rho}$, we have the inequality

$$
|y|_{\rho^{\epsilon}} \leq|y|^{1-\epsilon}|y|_{\rho}^{\epsilon}
$$

since it is actually an equality for monomials.

Given $x \in B$, choose $y \in W_{n}$ and $z \in T_{n, \rho}$, both reducing to $x$ in $A$, with $|y|=|x|_{A}$ and $|z|_{\rho}=|x|_{B}$. By Lemma 2.4.1, we can find $u \in T_{n, \rho}$, also reducing to $x$ in $A$, with $|u| \leq|y|$ and $|u|_{\rho} \leq|z|_{\rho}$. We then have

$$
|u|_{\rho^{\epsilon}} \leq|u|^{1-\epsilon}|u|_{\rho}^{\epsilon}
$$

Put $C=f\left(T_{n, \rho^{\epsilon}}\right)$; then the residue norm $|\cdot|_{C}$ induced by the spectral norm on $T_{n, \rho^{\epsilon}}$ satisfies

$$
|x|_{C} \leq|x|_{A}^{1-\epsilon}|x|_{B^{\prime}}^{\epsilon}
$$

for all $x \in B$. Replacing $x$ by $x^{i}$, taking $i$-th roots and taking limits as $i \rightarrow \infty$ yields

$$
|x|_{\sup , C} \leq|x|_{\sup , A}^{1-\epsilon}|x|_{\sup , B^{\prime}}^{\epsilon}
$$

Since $|x|_{\text {sup }, B^{\prime}} \leq|x|_{\sup , B}$, this yields the desired result.

\subsection{Robba rings}

The Robba ring is typically used to study $p$-adic differential equations over a field. We will generalize its definition so that it can be constructed not just over a field, but over a reduced affinoid or dagger algebra. (It may be possible to avoid the reducedness hypothesis, but we have no need to do so here.) 
Definition 2.5.1. Let $A$ be a reduced affinoid algebra. For each $r>0$, let $\mathcal{R}_{A, r}$ denote the set of formal (bidirectional) power series $x=\sum_{i \in \mathbb{Z}} x_{i} t^{i}$, with $x_{i} \in A$, such that

$$
\lim _{i \rightarrow-\infty} v_{A}\left(x_{i}\right)+r i=\infty, \quad \liminf _{i \rightarrow+\infty} \frac{v_{A}\left(x_{i}\right)}{i} \geq 0
$$

an equivalent condition is that $\lim _{i \rightarrow \pm \infty} v_{A}\left(x_{i}\right)+s i=\infty$ for $0<s \leq r$. This set forms a ring under the usual multiplication law for power series. For $0<s \leq r$, we define the valuation $w_{A, s}$ on $\mathcal{R}_{A, r}$ by the formula

$$
w_{A, s}(x)=\min _{i}\left\{v_{A}\left(x_{i}\right)+s i\right\}
$$

then $\mathcal{R}_{A, r}$ is complete for the Fréchet topology defined by these valuations (that is, any sequence which is Cauchy with respect to each $w_{A, s}$ is convergent). The union $\mathcal{R}_{A}=\cup_{r>0} \mathcal{R}_{A, r}$ is called the Robba ring of $A$; we equip it with the direct limit of the Fréchet topologies on the $\mathcal{R}_{A, r}$.

Remark 2.5.2. Robba rings are not as well-behaved as the other rings we have considered so far; for instance, the Robba ring over $K$ is not noetherian. By a theorem of Lazard [La], it does however have the Bézout property: every finitely generated ideal over $\mathcal{R}_{K}$ (or $\mathcal{R}_{K, r}$ ) is principal. Over a Bézout ring, any finitely presented projective module, or any finitely generated locally free module, is actually free.

Extending this definition to the dagger case is a bit tricky; the most straightforward extension is not the one that we really need (see Remark 2.5.7).

Definition 2.5.3. Let $A$ be a reduced dagger algebra. Define the Robba ring $\mathcal{R}_{A}$ over $A$ as the ring of formal series $x=\sum_{i \in \mathbb{Z}} x_{i} t^{i}$ over $A$ with the following property: for each sufficiently small $r>0$, there exists a fringe algebra $B$ such that the $x_{i}$ belong to $B$ and

$$
\lim _{i \rightarrow \pm \infty} v_{B}\left(x_{i}\right)+r i=\infty
$$

or equivalently, $x_{i} p^{\lfloor r i\rfloor}$ converges to zero as $i \rightarrow \pm \infty$ in the fringe topology of $A$.

In practice, it is sometimes easier to check the following condition for membership in the Robba ring of a dagger algebra.

Proposition 2.5.4. Let $A$ be a reduced dagger algebra, and let $\widehat{A}$ be the completion of $A$ for the affinoid topology. Suppose $x=\sum_{i} x_{i} t^{i} \in \mathcal{R}_{\widehat{A}, r}$ satisfies $x_{i} \in A$ for all $i$. If the condition that $x_{i} p^{\lfloor s i\rfloor} \rightarrow 0$ as $i \rightarrow \pm \infty$ in the fringe topology of $A$ holds for $s=r$, it holds also when $0<s<r$.

Proof. Let $B$ be a fringe algebra in which $x_{i} p^{\lfloor r i\rfloor} \rightarrow 0$ as $i \rightarrow \pm \infty$. Then we also have $x_{i} p^{\lfloor s i\rfloor} \rightarrow 0$ in $B$ as $i \rightarrow-\infty$. On the other hand, by Proposition 2.4.2, for any rational $\epsilon \in(0,1]$, we can find a fringe algebra $C$ such that

$$
\left|x_{i}\right|_{\sup , C} \leq\left|x_{i}\right|_{\sup , A}^{1-\epsilon}\left|x_{i}\right|_{\sup , B}^{\epsilon} \quad \text { for all } i .
$$


If we choose $\epsilon<s / r$, we may then write

$$
p^{-\lfloor s i\rfloor}\left|x_{i}\right|_{\mathrm{sup}, C} \leq\left(p^{-\lfloor(s-\epsilon r) i\rfloor}\left|x_{i}\right|_{\sup , A}^{1-\epsilon}\right)\left(p^{-\lfloor\epsilon r i\rfloor}\left|x_{i}\right|_{\mathrm{sup}, B}^{\epsilon}\right) ;
$$

the right side tends to 0 as $i \rightarrow \infty$, so the left side does as well. This yields the desired result.

Corollary 2.5.5. Let $A$ be a reduced dagger algebra, and let $\widehat{A}$ be the completion of $A$ for the affinoid topology. Let $x=\sum_{i \in \mathbb{Z}} x_{i} t^{i}$ be a formal series with coefficients in $A$. Then $x \in \mathcal{R}_{A}$ if and only if $x \in \mathcal{R}_{\widehat{A}}$ and for some $r>0, x_{i} p^{\lfloor r i\rfloor} \rightarrow 0$ as $i \rightarrow \pm \infty$ in the fringe topology of A.

This means checking convergence in a Robba ring over a dagger algebra is typically a two-step process: one first checks convergence over the completion, then one checks the condition on the fringe topology.

Remark 2.5.6. We mention an example in passing (that will not be used later). For $A=K\langle x\rangle^{\dagger}$, it can be shown that a series $\sum_{i \in \mathbb{Z}} a_{i} t^{i}$ belongs to $\mathcal{R}_{A}$ if and only if there exist some $\delta>1$ and $\epsilon>0$ such that the series converges for $|x| \leq \delta, \delta^{-1} \leq|t|<1$ and $\left|x^{\epsilon} t\right|<1$.

Remark 2.5.7. Do not confuse $\mathcal{R}_{A}$ with the the direct limit of $\mathcal{R}_{B}$ over the fringe algebras $B$ of $A$, where $\mathcal{R}_{B}$ is defined by viewing $B$ as an affinoid. (The latter is obtained by reversing the order of the quantifiers "for $r>0$ sufficiently small" and "there exists a fringe algebra $B$ " in Definition 2.5.3.) This ring is strictly smaller than $\mathcal{R}_{A}$ : for instance, for $A=K\langle x\rangle^{\dagger}$, $1-x t$ is a unit in $\mathcal{R}_{A}$ but not in $\mathcal{R}_{B}$ for any fringe algebra $B$.

Definition 2.5.8. For $A$ a reduced dagger algebra, we define $w_{r}$ on $\mathcal{R}_{A}$ (or rather, on the subring where it makes sense) by restriction from $\mathcal{R}_{\widehat{A}}$. For $B$ a fringe algebra of $A$, we also define $w_{B, r}$ on the subring where it makes sense. We topologize $\mathcal{R}_{A}$ as follows: the sequence $\left\{x_{n}\right\}$ converges if and only if the $w_{B, r}\left(x_{n}\right)$ are defined and tend to $\infty$ for some $B$ and $r$, and for sufficiently small $s$ (not depending on $n$ ), the $w_{s}\left(x_{n}\right)$ are defined and tend to $\infty$. Equivalently (as in the proof of Proposition 2.5.4), for each sufficiently small $r$, there exists a fringe algebra $B$ such that the $w_{B, r}\left(x_{n}\right)$ are defined and tend to $\infty$.

Definition 2.5.9. In the notation of a Robba ring, we use the superscript "+" to denote the subring consisting of power series with only nonnegative powers of $t$. We call this subring the plus part.

Definition 2.5.10. In the notation of a Robba ring (or plus part), we use the superscript "int" to denote the subring consisting of series with integral coefficients. We call this subring the integral subring. Note that this subring can be topologically characterized, as the set of $x$ for which the sequence $\left\{\left(c x^{a}\right)^{n}\right\}_{n=1}^{\infty}$ converges to zero for any $c \in K$ with $|c|<1$ and any positive integer $a$. Consequently, any continuous map between Robba rings induces a continuous map on integral subrings. Again, the subset of the integral subring consisting of topologically nilpotent elements is an ideal; we call the quotient by this ideal the reduction of the Robba ring. It is isomorphic to the Laurent series ring $R((t))$, where $R$ is the reduction of $A$. 
We will also need a multidimensional version of this construction.

Definition 2.5.11. Let $T=\left\{t_{1}, \ldots, t_{n}\right\}$ be a finite set and $A$ a reduced affinoid algebra. We then define $\mathcal{R}_{A, r}^{T}$ as the set of formal sums $\sum_{I} x_{I} t^{I}$, for $I$ ranging over $\mathbb{Z}^{n}$, such that for $0<s_{1}, \ldots, s_{n} \leq r$

$$
\lim _{I} v_{A}\left(x_{I}\right)+s_{1} i_{1}+\cdots+s_{n} i_{n}=\infty .
$$

(That is, the quantity on the left takes values below any given cutoff for only finitely many I.) We define $\mathcal{R}_{A}^{T}$ as the union of the $\mathcal{R}_{A, r}^{T}$. (If $T$ is empty, we put $\mathcal{R}_{A}^{T}=A$.) Again, each $\mathcal{R}_{A, r}^{T}$ has a natural Fréchet topology, and we topologize $\mathcal{R}_{A}^{T}$ as the direct limit thereof. To define $\mathcal{R}_{A}^{T}$ for $A$ a reduced dagger algebra, we require that for each tuple of $s_{1}, \ldots, s_{n}>0$ with $\max _{j}\left\{s_{j}\right\}$ sufficiently small, we have

$$
\lim _{I} v_{B}\left(x_{I}\right)+s_{1} i_{1}+\cdots+s_{n} i_{n}=\infty
$$

for some fringe algebra $B$; again, for series in $\mathcal{R}_{\widehat{A}}^{T}$ it is enough to check this for a single tuple. We topologize as in Definition 2.5.8

One immediate benefit of the multidimensional construction (besides its ultimate value in computing cohomology with compact supports in Chapter [8) is that it allows us to interpret products of Robba rings. That is, the category of topological rings isomorphic to $\mathcal{R}_{A}^{S}$ for some finite set $S$ and some reduced affinoid (resp. dagger) algebra $A$, with continuous $K$-algebra morphisms, has pairwise products given by

$$
\mathcal{R}_{A}^{S} \widehat{\otimes} \mathcal{R}_{B}^{T}=\mathcal{R}_{A \widehat{\otimes} B}^{S \cup T}
$$

Remark 2.5.12. Although Robba rings are never noetherian (see Remark 2.5.2), as noted above, their integral subrings are better behaved. For instance, $\mathcal{R}_{K}^{\text {int }}$ is a discrete valuation ring with residue field $k((t))$. More generally, we expect that $\mathcal{R}_{A}^{T \text {,int }}$ is always noetherian for $A$ a reduced affinoid or dagger algebra, though this assertion is a bit subtle in case $T$ has more than one element. For instance, if $T=\{t, u\}$, then $\mathcal{R}_{K}^{T, \text { int }} / \pi \mathcal{R}_{K}^{T \text {,int }}$ consists of formal Laurent series $\sum_{i, j \in \mathbb{Z}} c_{i, j} t^{i} u^{j}$ such that for each $a, b>0$, there exist only finitely many pairs $(i, j)$ with $c_{i, j} \neq 0$ and $i+a j<b$. This ring includes the usual double Laurent series ring $k \llbracket t, u \rrbracket\left[t^{-1}, u^{-1}\right]$ but is somewhat bigger, and its noetherianness is not immediately evident.

Lemma 2.5.13. Let $A$ be a reduced dagger algebra. Suppose that $x \in \mathcal{R}_{A} \cap \mathcal{R}_{B, r}$ for some fringe algebra $B$ and some $r>0$, and that $w_{r}(x)>0$. Then there exists another fringe algebra $C$ of $A$ such that $w_{C, r}(x)>0$.

Proof. Write $x=\sum_{i} x_{i} t^{i}$; then already for all but finitely many $i$, we have $w_{B, r}\left(x_{i} t^{i}\right)>0$, and so $w_{C, r}\left(x_{i} t^{i}\right)>0$ for any fringe algebra $C$ containing $B$. To get the inequality for each of the finitely many remaining $i$, apply Proposition 2.4 .2 . 


\section{$3 \quad$ Frobenius and differential structures}

Overconvergent $F$-isocrystals on smooth $k$-schemes are essentially modules over certain dagger algebras equipped with "Frobenius" and "differential" structures. In this chapter, we review the notions that make it possible to describe these extra structures, culminating in the notion of $(\sigma, \nabla)$-modules and their cohomology.

\subsection{Differentials}

Definition 3.1.1. In order to make uniform statements, we adopt the following ad hoc definition. An eligible category is the category of one of the following types of objects.

- Affinoid algebras and $K$-algebra morphisms.

- Dagger algebras and $K$-algebra morphisms.

- Multidimensional Robba rings over reduced affinoid algebras, and continuous $K$-algebra morphisms.

- Multidimensional Robba rings over reduced dagger algebras, and continuous $K$-algebra morphisms.

- Plus parts of the rings in the previous two categories.

Definition 3.1.2. Let $\mathcal{C}$ be an eligible category. Let $R$ be an object in $\mathcal{C}$, and let $J$ be the kernel of the multiplication map $R \widehat{\otimes} R \rightarrow R$ (with the subspace topology). Define the module of continuous differentials of $R$ as

$$
\Omega_{R / K}^{1}=J / J^{2}
$$

equipped with the quotient topology and the continuous $K$-linear derivation $d: R \rightarrow \Omega_{R / K}^{1}$ sending $x$ to $1 \otimes x-x \otimes 1$. Then $\Omega_{R / K}^{1}$ is universal for continuous $K$-linear derivations from $A$ into separated topological $A$-modules. As usual, we write $\Omega_{R / K}^{i}$ for the exterior power $\wedge^{i} \Omega_{R / K}^{1}$ over $R$, and $d$ for the map $\Omega_{R / K}^{i} \rightarrow \Omega_{R / K}^{i+1}$ induced by the canonical derivation $d: R \rightarrow \Omega_{R / K}^{1}$.

It is easily checked that $\Omega_{T_{n} / K}^{1}$ and $\Omega_{W_{n} / K}^{1}$ are freely generated by $d x_{1}, \ldots, d x_{n}$. It follows that $\Omega_{A / K}^{1}$ is finitely generated whenever $A$ is an affinoid or dagger algebra; if $A$ is regular, then $\Omega_{A / K}^{1}$ is also locally free, hence projective. Similarly, one verifies that for $A$ a reduced affinoid or dagger algebra, $\Omega_{\mathcal{R}_{A}^{T} / K}^{1}$ is isomorphic to the direct sum of $\mathcal{R}_{A}^{T} \otimes_{A} \Omega_{A / K}^{1}$ with the $\mathcal{R}_{A}^{T}$-module freely generated by $T$. (For instance, $\Omega_{\mathcal{R}_{K} / K}^{1}$ is the free module generated by $d t$.) In particular, if $A$ is regular, then $\Omega_{\mathcal{R}_{A}^{T} / K}^{1}$ is also finite locally free.

Definition 3.1.3. If $A$ is a subring of $B$, we define the relative module of continuous differentials $\Omega_{B / A}^{1}$ as the quotient of $\Omega_{B / K}^{1}$ by the submodule generated by $d a$ for all $a \in A$. 
We will be particularly interested in the relative construction when $B=\mathcal{R}_{A}^{T}$; in this case we can write

$$
d x=\sum_{t \in T} \frac{\partial x}{\partial t} \otimes d t .
$$

Proposition 3.1.4. Let $A$ be an affinoid or dagger algebra. Given $x \in \mathcal{R}_{A}^{T}$, write $x=\sum_{i} c_{i} t^{i}$ for $c_{i} \in \mathcal{R}_{A}^{T \backslash\{t\}}$. Then:

(a) The kernel of $\frac{\partial}{\partial t}$ consists of those $x$ with $c_{i}=0$ for $i \neq 0$.

(b) The image of $\frac{\partial}{\partial t}$ consists of those $x$ with $c_{-1}=0$.

(c) The induced map $\mathcal{R}_{A}^{T} /\left(\operatorname{ker} \frac{\partial}{\partial t}\right) \rightarrow \operatorname{im} \frac{\partial}{\partial t}$ is a homeomorphism.

Proof. (a) Obvious.

(b) It suffices to check that the image includes every $x$ for which $c_{i}=0$ for $i \geq-1$, and every $x$ for which $c_{i}=0$ for $i \leq-1$. Write $T=\left\{t_{1}, \ldots, t_{n}\right\}$ with $t_{1}=t$, and write $x=\sum_{I} a_{I} t^{I}$. Then we are given

$$
a_{I} p^{\left\lfloor s_{1} i_{1}+\cdots+s_{n} i_{n}\right\rfloor} \rightarrow 0 \quad \forall s_{1}, \ldots, s_{n} \in(0, r]
$$

and need to deduce that

$$
a_{I} p^{\left\lfloor s_{1}^{\prime}\left(i_{1}+1\right)+\cdots+s_{n} i_{n}-\log _{p}\left|i_{1}+1\right|\right\rfloor} \rightarrow 0 \quad \forall s_{1}^{\prime}, s_{2}, \ldots, s_{n} \in\left(0, r^{\prime}\right]
$$

for some $r^{\prime}<r$. In the first case, we get the second estimate from the first by taking $s_{1}<s_{1}^{\prime}$ and $r^{\prime}=r$; in the second case, we get it by taking $s_{1}>s_{1}^{\prime}$ and $r^{\prime}<r$.

(c) It is clear that the induced map is continuous; the proof of (b) shows that its inverse is also continuous.

\subsection{Algebras of MW-type and Frobenius lifts}

Definition 3.2.1. We say an affinoid or dagger algebra $A$ is of $M W$-type (short for "of Monsky-Washnitzer type") if $A^{\text {int }} / \pi A^{\text {int }}$ is a smooth $k$-algebra. In particular, $A$ is regular, and its reduction coincides with $A^{\text {int }} / \pi A^{\text {int }}$.

In the terminology of [MW], a dagger algebra of MW-type is a "formally smooth, weakly complete, weakly finitely generated algebra".

Algebras of MW-type are known to have good lifting properties (see for instance $[\mathrm{Ar}]$ ). For one, given any smooth $k$-algebra $R$, there exist an affinoid algebra and a dagger algebra of MW-type with reduction $R$, and both are unique up to noncanonical isomorphism. For another, if $A$ and $B$ are affinoid/dagger algebras of MW-type with reductions $R$ and $S$, then any morphism $R \rightarrow S$ lifts to a morphism $A \rightarrow B$. These lifts retain many good properties of the original map: e.g., they are finite flat if the original map is (see Lemma 2.2.3). 
Remark 3.2.2. One example of the lifting properties is Noether normalization: any affinoid (resp. dagger) algebra of MW-type whose special fibre is purely of dimension $n$ (e.g., irreducible) can be written as a finite extension of $T_{n}$ (resp. $W_{n}$ ), by applying classical Noether normalization and lifting the resulting map from $k\left[x_{1}, \ldots, x_{n}\right]$ into the reduction.

Another key example is given by the Frobenius lifts.

Definition 3.2.3. For $A$ a ring in any of the categories in Definition 3.1.1 define a Frobenius lift on $A$ to be a continuous homomorphism $\sigma: A \rightarrow A$ which is $\sigma_{K^{-}}$-linear over $K$ and which acts on the reduction of $A$ via the $q$-power Frobenius map. If we write $A_{\sigma_{K}}$ for $A$ with its $K$-action funneled through $\sigma_{K}$, then a Frobenius lift is given by a $K$-linear map $A_{\sigma_{K}} \rightarrow A$ lifting the relative Frobenius on reductions. In case $A$ is an affinoid or dagger algebra of MW-type, the existence of a Frobenius lift is thus guaranteed by the good lifting properties discussed above. If $A$ is a Robba ring or plus part over an affinoid or dagger algebra $B$ of MW-type, one can extend to $A$ any Frobenius lift $\sigma_{B}$ on $B$ by setting

$$
\sum_{I} b_{I} t^{I} \mapsto \sum_{I} b_{I}^{\sigma_{B}} t^{q i}
$$

We call this the standard extension of $\sigma_{B}$.

Remark 3.2.4. Note that for $A$ a dagger algebra of MW-type and $\sigma$ a Frobenius lift on $A, \sigma$ is finite flat because the Frobenius map on the reduction of $A$ (that being a smooth $k$-scheme) is finite flat.

Remark 3.2.5. Beware that a Frobenius lift on a dagger algebra does not act on individual fringe algebras. For instance, the Frobenius lift on $T_{n}$ given by $\sum c_{I} t^{I} \mapsto \sum c_{I}^{\sigma_{K}} t^{q I}$ carries the fringe algebra $T_{n, \rho}$ into the larger fringe algebra $T_{n, \rho^{1 / q}}$.

By restricting to dagger algebras of MW-type, we can invoke the following result $\mathrm{MW}$. Theorem 8.3] (see also [Be5, Proposition 3.6]).

Proposition 3.2.6. Let $f: R \rightarrow S$ be a morphism of dagger algebras of $M W$-type, and suppose $S$ is finite and locally free over $R$. Then there is an $R$-linear map of complexes Trace : $\Omega_{S / K} \rightarrow \Omega_{R / K}$ such that Trace of is multiplication by the degree of $S$ over $R$.

Here $R$-linearity means that

$$
\operatorname{Trace}(\omega \wedge \eta)=\omega \wedge \operatorname{Trace}(\eta) \quad \omega \in \Omega_{R / K}, \eta \in \Omega_{S / K} .
$$

Remark 3.2.7. We believe the same result is true in any eligible category, but the proof of [MW, Theorem 8.3] uses the noetherian property of dagger algebras, and so does not apply to Robba rings. (The proof of [Be5, Proposition 3.6] relies on [MW, Theorem 8.3], so is of no help either.) However, we can construct the trace "by hand" in two particular instances of interest: 
(a) In case $R=\mathcal{R}_{A}$ and $S=\mathcal{R}_{B}$ where $A$ and $B$ are reduced dagger algebras of MW-type and $B / A$ is finite projective. In this case we may simply apply the trace from $B$ to $A$ coefficientwise.

(b) In case $R=\mathcal{R}_{A}$ and $S=\mathcal{R}_{A} \otimes_{\mathcal{R}_{A}^{\text {int }}} \mathcal{R}^{\prime}$ where $\mathcal{R}^{\prime}$ is a finite Galois extension of $\mathcal{R}_{A}^{\text {int }}$. In this case, we may simply sum over $\operatorname{Aut}\left(\mathcal{R}^{\prime} / \mathcal{R}_{A}^{\text {int }}\right)$ to obtain the trace.

\section{$3.3(\sigma, \nabla)$-modules}

We now introduce the coefficient objects in Monsky-Washnitzer cohomology.

Definition 3.3.1. Let $R$ be a ring of one of the types described in Definition 3.1.1 equipped with a Frobenius lift $\sigma$, and let $A$ be a subring preserved by $\sigma$. (If $A$ is not specified, take it to be $K$.) Define a $(\sigma, \nabla)$-module over $R$, relative to $A$, as a finite locally free module $M$ over $R$ equipped with:

(a) a Frobenius structure: an additive, $\sigma$-linear map $F: M \rightarrow M$ (that is, $F(r \mathbf{v})=$ $r^{\sigma} F(\mathbf{v})$ for $r \in R$ and $\left.\mathbf{v} \in M\right)$ such that the induced $R$-linear map $\sigma^{*} M \rightarrow M$ is an isomorphism;

(b) an integrable connection: an additive, $A$-linear map $\nabla: M \rightarrow M \otimes_{R} \Omega_{R / A}^{1}$ satisfying the Leibniz rule: $\nabla(r \mathbf{v})=r \nabla(\mathbf{v})+\mathbf{v} \otimes d r$ for $r \in R$ and $\mathbf{v} \in M$, and such that, if we write $\nabla_{n}$ for the induced map $M \otimes_{R} \Omega_{R / A}^{n} \rightarrow M \otimes_{R} \Omega_{R / A}^{n+1}$, we have $\nabla_{n+1} \circ \nabla_{n}=0$ for all $n \geq 0$

subject to the compatibility condition

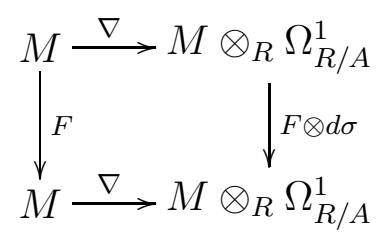

Remark 3.3.2. Note that the integrability condition $\nabla_{n+1} \circ \nabla_{n}=0$ is redundant if $\Omega_{R / A}^{1}$ is projective of rank 1, e.g., if $R$ is a one-dimensional Robba ring over $A$. In any case, it suffices to check that $\nabla_{1} \circ \nabla_{0}=0$.

Remark 3.3.3. The compatibility condition means simply that the map $\sigma^{*} M \rightarrow M$ induced by $F$ is horizontal for the connection.

It is worth noting that over a regular affinoid or dagger algebra, the hypothesis of local freeness in the definition of a $(\sigma, \nabla)$-module can be dropped.

Lemma 3.3.4. Let $A$ be a regular affinoid or dagger algebra, and let $M$ be a finitely generated A-module equipped with a connection $\nabla: M \rightarrow M \otimes \Omega_{A / K}^{1}$. Then $M$ is locally free. 
Proof. The proof is basically the same as for the analogous complex analytic statement (see, e.g., [P Lemme 10.3.1]). It suffices to check that for each maximal ideal $\mathfrak{a}$ of $A, M \otimes A_{\mathfrak{a}}$ is free, where $A_{\mathfrak{a}}$ is the local ring of $A$ at $\mathfrak{a}$. For $r \in A_{\mathfrak{a}}$, let $\operatorname{ord}(r)$ denote the order of vanishing of $r$ at $\mathfrak{a}$.

Choose $\mathbf{v}_{1}, \ldots, \mathbf{v}_{n} \in M$ whose images in $M / \mathfrak{a} M$ form a basis of that vector space over $A / \mathfrak{a}$. By Nakayama's lemma, $\mathbf{v}_{1}, \ldots, \mathbf{v}_{n}$ generate $M \otimes A_{\mathfrak{a}}$, and we must only check that the equation $\sum c_{i} \mathbf{v}_{i}=0$, with $c_{i} \in A_{\mathfrak{a}}$, implies that the $c_{i}$ are all zero. Suppose that this is not the case; choose a counterexample that minimizes $\min _{i}\left\{\operatorname{ord}\left(c_{i}\right)\right\}$. This minimum cannot be zero, since the $\mathbf{v}_{i}$ are linearly independent in $M / \mathfrak{a} M$.

Choose vector fields $\partial_{1}, \ldots, \partial_{d}$ on Maxspec $A$ which at $\mathfrak{a}$ form a basis of the tangent space there, and let $N_{j}$ be the contraction of $\nabla$ with $\partial_{j}$. Then for each $j$,

$$
0=\sum_{i}\left(\partial_{j} c_{i}\right) \mathbf{v}_{i}+c_{i}\left(N_{j} \mathbf{v}_{i}\right)
$$

Suppose without loss of generality that $m_{1}=\min _{i}\left\{m_{i}\right\}$; then we can choose $j$ so that $\partial_{j} c_{1}$ is nonzero and ord $\left(\partial_{j} c_{i}\right)<m_{1}$. On the other hand, the order of vanishing of the coefficient of $\mathbf{v}_{1}$ in $c_{i}\left(N_{j} \mathbf{v}_{i}\right)$ is at least $m_{i} \geq m_{1}$. We thus obtain a new linear combination of the $\mathbf{v}_{i}$ which vanishes, and the minimum order of vanishing of a coefficient has decreased, contradicting the choice of the $c_{i}$.

We conclude that the equation $\sum c_{i} \mathbf{v}_{i}=0$ has only the trivial solution, so that $M \otimes A_{\mathfrak{a}}$ is freely generated by the $\mathbf{v}_{i}$. This proves the desired result.

\subsection{Cohomology of $(\sigma, \nabla)$-modules}

Definition 3.4.1. Let $M$ be a $(\sigma, \nabla)$-module over a ring $R$ (again of the type described in Definition 3.1.1) relative to a subring $A$. Thanks to the integrability condition, we have a de Rham complex associated to $M$ :

$$
\cdots \rightarrow M \otimes \Omega_{R / A}^{i} \stackrel{\nabla_{i}}{\rightarrow} M \otimes \Omega_{R / A}^{i+1} \rightarrow \cdots
$$

Define the cohomology of $M$ as the cohomology of this complex:

$$
H^{i}(M)=\frac{\operatorname{ker}\left(M \otimes \Omega_{R / A}^{i} \stackrel{\nabla_{i}}{\rightarrow} M \otimes \Omega_{R / A}^{i+1}\right)}{i m\left(M \otimes \Omega_{R / A}^{i-1} \stackrel{\nabla_{i-1}}{\rightarrow} M \otimes \Omega_{R / A}^{i}\right)} .
$$

Definition 3.4.2. If $f: R \rightarrow S$ is an $A$-algebra homomorphism and $S$ can be equipped with a Frobenius lift compatible with the one given on $R$, we define the pullback $f^{*} M$ of a $(\sigma, \nabla)$-module $M$ over $R$ as the module $M \otimes_{R} S$ with the natural extra structures. If $f: S \rightarrow R$ is an injective $A$-algebra homomorphism such that $R$ is finite étale over $S$, we define the pushforward $f_{*} M$ as $M$ itself viewed as an $S$-module. (The terminology looks backward on the algebra level, but becomes correct when we pass to geometry and reverse arrows.) 
We have the following relationships between cohomology under pullback and pushforward.

Proposition 3.4.3. Let $f: R \rightarrow S$ be an injective A-algebra homomorphism such that $S$ is a finite projective $R$-module, and suppose $\sigma$ is a Frobenius lift on $S$ mapping $R$ and $A$ into themselves. Also assume either that $R$ and $S$ are dagger algebras of $M W$-type, or that one of the conditions of Remark 3.2.7 applies. Then for any $(\sigma, \nabla)$-module $M$ over $R$, relative to $A$, there is an injection $H^{i}(M) \hookrightarrow H^{i}\left(f^{*} M\right)$ for all $i$, whose image is that of a canonical projector on $H^{i}\left(f^{*} M\right)$.

Proof. By Proposition 3.2.6 or Remark 3.2.7 there is a trace map Trace $: \Omega_{S / K} \rightarrow \Omega_{R / K}$ such that Trace of is multiplication by a positive integer, and likewise when $K$ is replaced by $A$. Tensoring with $M$ and taking cohomology yields maps $H^{i}(M) \rightarrow H^{i}\left(f^{*} M\right) \rightarrow H^{i}(M)$ whose composition is multiplication by a positive integer, yielding the desired result.

Remark 3.4.4. Note that the above argument only uses the $A$-linearity of $f$ in order to conclude that the maps defined between $\Omega_{R / K}$ and $\Omega_{S / K}$ descend to $\Omega_{R / A}$ and $\Omega_{S / A}$. This holds even if we only assume $f(A) \subseteq A$; in particular it holds for $f=\sigma$ when $A$ is a dagger algebra of MW-type (so that $\sigma$ is finite flat). We thus conclude that $H^{i}(M) \rightarrow H^{i}\left(\sigma^{*} M\right)$ is injective; since the Frobenius structure on $M$ consists of an isomorphism of $\sigma^{*} M$ with $M$, we conclude that the induced ( $A$-semilinear) action of $F$ on $H^{i}(M)$ is also injective.

Proposition 3.4.5. Let $R$ be a dagger algebra, let $f: R \rightarrow S$ be a finite étale extension of $R$, and let $M$ be a $(\sigma, \nabla)$-module on $S$. Then there is a canonical isomorphism $H^{i}(M) \rightarrow$ $H^{i}\left(f_{*} M\right)$ for all $i$.

Proof. In fact, there is a canonical isomorphism of the underlying de Rham complexes: for each $i$, one has a canonical isomorphism

$$
S \otimes_{R} \Omega_{R / K}^{i} \cong \Omega_{S / K}^{i}
$$

and this remains an isomorphism after tensoring with $M$.

\section{Rigid cohomology}

In this chapter, we review Berthelot's theory of rigid cohomology, and recall its connection to the Monsky-Washnitzer theory (see especially Remarks 4.2.5 and 4.3.3). We remind the reader of a useful analogy: Monsky-Washnitzer cohomology is analogous to the "naive" algebraic de Rham cohomology of smooth affine schemes, which is simply the cohomology of the complex of Kähler differentials of the coordinate ring. Rigid cohomology is analogous to the "correct" definition of algebraic de Rham cohomology, in which one (locally) embeds a given variety into a smooth variety, considers the formal neighborhood, and then computes hypercohomology of the resulting de Rham complex. The choice of the embedding does not 
matter because any two such embeddings end up being homotopy-equivalent on the level of de Rham complexes.

Our treatment here is by no means definitive. For further information, a good introduction is Be2], but it contains very few proofs. A more comprehensive development in the case of constant coefficients is Be5; most of the arguments there can be extended to nonconstant coefficients relatively easily. The notion of isocrystals is comprehensively developed in the unpublished [Be4]. Of course, all of this theory is ultimately subsumed into the theory of arithmetic $\mathcal{D}$-modules anyway, for which see [Be7] for an introduction.

\subsection{Some rigid geometry}

We begin with some preliminaries from rigid geometry, namely Raynaud's construction [R] of the "generic fibre" of a formal $\mathcal{O}$-scheme. The canonical reference for this subject is of course [BGR, Part C], to which we refer for such notions as rigid analytic spaces, admissible open subsets, and sheaf cohomology.

Definition 4.1.1. Let $P$ be a formal $\mathcal{O}$-scheme which is separated and of finite type. We define the generic fibre $\tilde{P}$ to be the set of closed formal subschemes of $P$ which are integral, finite and flat over $\mathcal{O}$. This set comes with a specialization map sp : $\tilde{P} \rightarrow P$ which associates to each subscheme its support.

For $P$ affine, we can write $P=\operatorname{Spf}(R)$ for some complete topologically finitely generated $\mathcal{O}$-algebra $R$, and identify $\tilde{P}$ with $\operatorname{Maxspec}\left(R \otimes_{\mathcal{O}} K\right)$. This gives $\tilde{P}$ the structure of a rigid analytic space; it can be shown that this structure does not depend on the choice of $R$. In particular, the structures glue to give $\tilde{P}$ the structure of a rigid analytic space for any $P$. (More precisely, $\tilde{P}$ is a locally $G$-ringed space for an appropriate Grothendieck topology.)

Definition 4.1.2. For $P$ a formal $\mathcal{O}$-scheme and $X$ a subscheme of the special fibre of $P$, we define the tube of $X$, denoted $] X\left[\right.$, to be the inverse image $\operatorname{sp}^{-1}(X)$.

We make the following definition following [Ki, Definition 2.3].

Definition 4.1.3. A rigid analytic space is quasi-Stein if it is the union of an increasing sequence $U_{1} \subseteq U_{2} \subseteq$. o of affinoid subdomains such that $\Gamma\left(U_{i}\right)$ is dense in $\Gamma\left(U_{i+1}\right)$ for all $i$.

The main result about quasi-Stein spaces is "Theorem B" [Ki, Satz 2.4].

Proposition 4.1.4. Let $\mathcal{G}$ be a coherent sheaf on a quasi-Stein space $X$. Then the cohomology groups $H^{i}(X, \mathcal{G})$ vanish for $i>0$.

\subsection{Overconvergent $F$-isocrystals}

In this section, we recall the notion of overconvergent $F$-isocrystals from $\mathrm{Be} 2$. These play the role of the coefficient objects in rigid cohomology; they are analogous to local systems 
in ordinary de Rham cohomology or lisse sheaves in étale cohomology. They are also closely related to the $(\sigma, \nabla)$-modules of the previous chapter; see Remark 4.2.5.

For simplicity, we restrict to the following situation. (One can generalize the definitions by a glueing construction.)

Hypothesis 4.2.1. Let $X$ be a $k$-scheme and let $\bar{X}$ be a compactification of $X$; put $Z=$ $\bar{X} \backslash X$. Suppose that there exists a closed immersion of $\bar{X}$ into a $\mathcal{O}$-formal scheme $P$ which is smooth in a neighborhood of $X$. Suppose further that there exists an isomorphism $\sigma$ of $P$ with itself that induces the action of $\sigma_{K}$ on $K$ and the $q$-power (absolute) Frobenius on $\bar{X}$, and fix a choice of $\sigma$.

Definition 4.2.2. Suppose that $P$ is affine. Define a strict neighborhood of $] X[$ in $] \bar{X}$ [ as an admissible open subset $V$ containing $] X$ [ such that for any affinoid $W$ contained in $] \bar{X}$, we can find sections $f_{1}, \ldots, f_{m} \in \Gamma(\tilde{P})$, whose images in $\Gamma(\bar{X})$ cut out $Z$, and some $\lambda<1$ such that

$$
W \cap\{x \in] \bar{X}\left[: \max _{i}\left\{\left|f_{i}(x)\right|\right\} \geq \lambda\right\} \subseteq W \cap V .
$$

For $P$ nonaffine, we make the same definition locally.

Definition 4.2.3. An overconvergent $F$-isocrystal $\mathcal{E}$ on $X$, relative to $K$, is a finite locally free $\mathcal{O}_{V}$-module on some strict neighborhood $V$ of $] X$, equipped with a connection $\nabla$ : $\mathcal{E} \rightarrow \mathcal{E} \otimes \Omega_{V}^{1}$ and an isomorphism $F: \sigma^{*} \mathcal{E} \rightarrow \mathcal{E}$ of modules with connection, subject to a convergence condition which will not be described in detail here (it asserts that the Taylor isomorphism is defined on a strict neighborhood of $] X\left[P \times_{\mathcal{O}} P\right)$. This definition appears to depend on the choice of $P$, but in fact there are canonical equivalences (via pullbacks, and relying on the convergence condition) between the categories constructed using different $P$.

Remark 4.2.4. We reiterate that the equivalences of categories between different choices of $P$ are homotopy equivalences on the level of de Rham complexes. It may be useful to imagine them as homotopy equivalences in the true topological sense; that is, there are maps between the two rigid analytic spaces whose compositions are close enough to being the identity maps that they can be "continuously deformed" into the identity map. For an example of this, see [MW, Theorem 5.5].

Remark 4.2.5. For $X$ smooth affine, the direct limit $A$ of $\Gamma\left(V, \mathcal{O}_{V}\right)$ over all embeddings $j:] X[\rightarrow V$ into a strict neighborhood is a dagger algebra of MW-type over $K$ with special fibre $X$. This turns out to yield an equivalence between the category of overconvergent $F$-isocrystals on $X$, relative to $K$, and the category of $(\sigma, \nabla)$-modules over $A$; the main point is that in this setting, the convergence condition is an automatic consequence of the presence of a Frobenius structure [Be4, Théorème 2.5.7]. This connection extends also to the cohomology level; see Remark 4.3 .3

\subsection{Rigid cohomology with and without supports}

We now recall Berthelot's definition of rigid cohomology, first without supports. 
Definition 4.3.1. The rigid cohomology of $\mathcal{E}$, notated $H_{\text {rig }}^{i}(X / K, \mathcal{E})$, is given by

$$
H_{\mathrm{rig}}^{i}(X / K, \mathcal{E})=H^{i}(] \bar{X}\left[, j^{\dagger}\left(\mathcal{E} \otimes \Omega_{V}\right)\right),
$$

where $j^{\dagger}$ denotes the direct limit over all inclusions $\left.j:\right] X[\hookrightarrow V$ into a strict neighborhood. This construction is independent of the choice of compactification; see [Be2, 2.3].

Remark 4.3.2. The notation is a little peculiar, because it is $\mathcal{E}$ and not $X$ that depends on the choice of $K$. It is set this way because in the case of constant coefficients, one omits $\mathcal{E}$ but still needs to keep track of the coefficient field.

Remark 4.3.3. Recall from Remark 4.2.5 that if $X$ is smooth affine, then an overconvergent $F$-isocrystal $\mathcal{E}$ on $X$ corresponds to a $(\sigma, \nabla)$-module $M$ over a dagger algebra $A$ of $\mathrm{MW}$ type with special fibre $X$. This comparison extends to cohomology: the rigid cohomology $H_{\text {rig }}^{i}(X / K, \mathcal{E})$ is canonically isomorphic to $H^{i}(M)$. See [Be2, Proposition 1.12] for a detailed discussion (in the constant coefficient case, but the general case is similar).

We next turn to cohomology with supports.

Definition 4.3.4. Let $\iota:] Z[\cap V \hookrightarrow V$ denote the obvious inclusion; note that $] Z[$ is an admissible open subset of $] \bar{X}$. For $\mathcal{F}$ a sheaf of abelian groups on $V$, put

$$
\underline{\Gamma}(\mathcal{F})=\operatorname{ker}\left(\mathcal{F} \rightarrow \iota_{*} \iota^{*} \mathcal{F}\right) ;
$$

this functor is left exact and admits right derived functors. For $\mathcal{F}$ coherent, by Theorem B we have $\mathbb{R}^{q} \iota_{*} \iota^{*} \mathcal{F}=0$ for $q>0$, so the total derived complex $\mathbb{R}^{q} \underline{\Gamma}(\mathcal{F})$ is isomorphic (in the derived category) to the two-term complex

$$
0 \rightarrow \mathcal{F} \rightarrow \iota_{*} \iota^{*} \mathcal{F} \rightarrow 0
$$

The rigid cohomology with compact supports of $\mathcal{E}$, notated $H_{c, \mathrm{rig}}^{i}(X / K, \mathcal{E})$, is given by

$$
H_{c, \text { rig }}^{i}(X / K, \mathcal{E})=H^{i}\left(V, \mathbb{R} \underline{\Gamma}\left(\mathcal{E} \otimes \Omega_{V}\right)\right) .
$$

In addition to being independent of the compactification, this construction is also independent of the choice of $V$; see [Be2, 3.3] and subsequent remarks.

Remark 4.3.5. As one might expect, there is a natural "forget supports" map

$$
H_{c, \text { rig }}^{i}(X / K, \mathcal{E}) \cong H_{\text {rig }}^{i}(X / K, \mathcal{E}),
$$

and this map is an isomorphism when $X$ is proper. We will not use either of these facts in what follows; however, they are crucial when considering the Weil conjectures, as in [Ke6]. 


\subsection{Additional properties}

We will use a few other formal properties of rigid cohomology, particularly in Chapter 9 as we prove our main results.

One has in rigid cohomology a notion of cohomology with support along a closed subscheme, as described in [Be5, 2.1]. Briefly, if $U$ is an open subscheme of $X$ and $Z=X \backslash U$, and $\mathcal{E}$ is an overconvergent $F$-isocrystal on $X$, there is a natural surjective map of sheaves from the sheaf $j^{\dagger}(\mathcal{E} \otimes \Omega$ ) to its "restriction to $U$ " (really, the limit of the direct images of its restrictions to strict neighborhoods of $] U[$ ). The cohomology of the kernel of this map is denoted $H_{Z \text {,rig }}^{i}(X / K, \mathcal{E})$. By construction, it fits into a long exact sequence with the cohomologies of $X$ and $U$; more careful consideration shows that one actually has a long exact sequence

$$
\cdots \rightarrow H_{T / K, \text { rig }}^{i}(X, \mathcal{E}) \rightarrow H_{Z / K, \text { rig }}^{i}(X, \mathcal{E}) \rightarrow H_{Z \backslash T, \mathrm{rig}}^{i}(X \backslash T / K, \mathcal{E}) \rightarrow \cdots
$$

whenever $T$ is a closed subscheme of $Z$. (See [Be5, Proposition 2.5] for the constant coefficient case; as usual, the general case is similar.)

In order for excision to be useful, one needs to have a Gysin map comparing $H_{Z \text {,rig }}^{i}(X / K, \mathcal{E})$ with $H_{\text {rig }}^{i}(Z / K, \mathcal{E})$, at least when $X$ and $Z$ are smooth. As it stands, one only has this with some additional restrictions, but this will be enough for our purposes.

Hypothesis 4.4.1. Suppose $Z \hookrightarrow X$ is a smooth pair, with $\operatorname{dim} Z \leq n$, admitting a lifting $\mathcal{Z} \hookrightarrow \mathcal{X}$ over $\mathcal{O}$, such that $\mathcal{Z}, \mathcal{X}$ are smooth over $\mathcal{O}$ and there exists an étale morphism $\mathcal{X} \rightarrow \operatorname{Spec} \mathcal{O}\left[x_{1}, \ldots, x_{d}\right]$ for some integer $d$, such that $\mathcal{Z}$ maps to the vanishing locus of $d-n$ of the $x_{i}$.

Under Hypothesis 4.4.1, Tsuzuki [T3, Theorem 4.1.1] constructs a Gysin isomorphism

$$
H_{Z, \text { rig }}^{i}(X / K, \mathcal{E}) \cong H_{\text {rig }}^{i-2 c}\left(Z / K,\left.\mathcal{E}\right|_{Z}\right)
$$

where $c=\operatorname{dim} X-\operatorname{dim} Z$.

In cohomology with compact supports, one has a similar excision sequence going the other way:

$$
\cdots \rightarrow H_{c, \text { rig }}^{i}(U, \mathcal{E}) \rightarrow H_{c, \text { rig }}^{i}(X, \mathcal{E}) \rightarrow H_{c, \text { rig }}^{i}(Z, \mathcal{E}) \rightarrow \cdots
$$

see [T3, Proposition 2.5.1]. As one expects, all three terms in this sequence are of the same type, so there is no need for a Gysin construction.

Finally, we will frequently compute by pushing forward along a finite étale morphism. This operation behaves as expected: in particular, one has the following relationship (see [T3, Corollaries 2.6.5 and 2.6.6]).

Proposition 4.4.2. Let $f: X \rightarrow Y$ be a finite étale morphism of separated $k$-schemes of $f$ nite type, and let $\mathcal{E}$ be an overconvergent $F$-isocrystal on $X$. Then for each $i$, there are canonical isomorphisms $H_{\text {rig }}^{i}(X / K, \mathcal{E}) \cong H_{\text {rig }}^{i}\left(Y / K, f_{*} \mathcal{E}\right)$ and $H_{c, \text { rig }}^{i}(X / K, \mathcal{E}) \cong H_{c, \text { rig }}^{i}\left(Y / K, f_{*} \mathcal{E}\right)$. 


\section{Relative local monodromy}

In this section, we concentrate on $(\sigma, \nabla)$-modules over (one-dimensional) Robba rings and their cohomology. We recall the notion of unipotence and the statement of the statement of the $p$-adic local monodromy theorem (Crew's conjecture) for $(\sigma, \nabla)$-modules over $\mathcal{R}_{K}$. We then formulate an analogue for a Robba ring over a dagger algebra, and apply this analogue to study the cohomology of a $(\sigma, \nabla)$-module over such a Robba ring.

\subsection{Unipotence and $p$-adic local monodromy}

Definition 5.1.1. Let $A$ be a reduced dagger algebra and let $M$ be a free $(\sigma, \nabla)$-module over the Robba ring $\mathcal{R}_{A}$, relative to $A$. Define a unipotent basis of $M$ to be a basis $\mathbf{v}_{1}, \ldots, \mathbf{v}_{n}$ for which $\nabla \mathbf{v}_{i} \in\left(\mathcal{R}_{A} \mathbf{v}_{1}+\cdots+\mathcal{R}_{A} \mathbf{v}_{i-1}\right) \otimes \frac{d t}{t}$ for all $i$. We say $M$ is unipotent if it admits a unipotent basis. (Note that the definition does not refer explicitly to the Frobenius structure.)

Note that the definition of unipotence does not depend on the implicit choice of the series parameter in the definition of $\mathcal{R}_{A}$; that is, it is invariant under pullback by any continuous automorphism of $\mathcal{R}_{A}$.

Over a field, the definitive statement about the property of unipotence is the $p$-adic local monodromy theorem ("Crew's conjecture"). This theorem has been proved independently by André [An], Mebkhout [Me2, and the author Ke1].

Theorem 5.1.2. Let $M$ be a free $(\sigma, \nabla)$-module over $\mathcal{R}_{K}$. Then there exist an integer $m \geq 0$, a finite unramified extension $K_{1}$ of $K_{0}=K^{\sigma^{-m}}$, a finite Galois (étale) extension $\mathcal{R}^{\prime}$ of $\mathcal{R}_{K_{1}}^{\text {int }}$, and a continuous $K_{1}$-algebra isomorphism of

$$
\mathcal{R}^{\prime \prime}=\mathcal{R}_{K_{1}} \otimes_{\mathcal{R}_{K_{1}}^{\text {int }}} \mathcal{R}^{\prime}
$$

with $\mathcal{R}_{K_{1}}$ such that $M \otimes \mathcal{R}^{\prime \prime}$ is unipotent.

Note that the conclusion does not depend on the choice of the isomorphism $\mathcal{R}^{\prime \prime} \cong \mathcal{R}_{K_{1}}$, only on the fact that such an isomorphism exists.

Proof. We explain here how to deduce the theorem from [Ke1, Theorem 6.12]. First recall that the category of $(\sigma, \nabla)$-modules over $\mathcal{R}_{K}$ is independent of the choice of $\sigma$; see for instance [Ke5, Definition 6.1.2]. We may thus assume that $\sigma$ is a power of a $p$-power Frobenius lift. (This step is needed because [Ke1 always assumes Frobenius lifts are powers of $p$-power Frobenius lifts. This can be avoided by instead invoking [Ke7, Theorem 7.2.5].)

Now by [Ke1, Theorem 6.12], $M$ becomes unipotent after tensoring up to the extension of $\mathcal{R}_{K}$ corresponding to a "nearly finite separable extension" of $k((t))$, i.e., a finite separable extension $L$ of $k_{0}((t))$ with $k_{0}=k^{q^{-m}}$ for some $m \geq 0$. There is no harm in enlarging $L$, so we may replace $L$ by its Galois closure over $k_{0}((t))$. By increasing $m$ (and correspondingly enlarging $L$ ), we can ensure that the residue field of $L$ is separable over $k_{0}$, and hence 
coincides with the integral closure of $k_{0}$ in $L$; call this field $k_{1}$. Let $K_{1}$ be the unramified extension of $K_{0}=K^{\sigma^{-m}}$ with residue field $k_{1}$. Since $\mathcal{R}_{K_{1}}^{\text {int }}$ is a henselian discrete valuation ring [Ke1, Lemma 3.9], we can uniquely lift $L$ to a finite étale extension $\mathcal{R}^{\prime}$ of $\mathcal{R}_{K_{1}}^{\text {int }}$. Since the residue field of $L$ coincides with $k_{1}$, we can find a continuous isomorphism $L \cong k_{1}((u))$ by the Cohen structure theorem; by lifting $u$, we obtain an isomorphism of

$$
\mathcal{R}^{\prime \prime}=\mathcal{R}_{K_{1}} \otimes_{\mathcal{R}_{K_{1}}^{\mathrm{int}}} \mathcal{R}^{\prime}
$$

with $\mathcal{R}_{K_{1}}$ such that $M \otimes \mathcal{R}^{\prime \prime}$ is unipotent, as desired.

The purpose of this section is to salvage Theorem 5.1 .2 in case $K$ is replaced by an integral dagger algebra of MW-type. For starters, we can apply Theorem 5.1.2 with $K$ replaced by the completion of Frac $A$ for the affinoid topology. (One might call the outcome of this application "generic quasi-unipotence".) However, one would like to perform a less drastic enlargement, replacing $A$ only by a localization. Happily, this is indeed possible.

Theorem 5.1.3. Let $A$ be an integral affinoid or dagger algebra of $M W$-type equipped with a Frobenius lift, and let $M$ be a free $(\sigma, \nabla)$-module over $\mathcal{R}_{A}$, relative to $A$. Then there exist a localization $B$ of $A$, an integer $m \geq 0$, a finite étale extension $B_{1}$ of $B_{0}=B^{\sigma^{-m}}$, a finite Galois (étale) extension $\mathcal{R}^{\prime}$ of $\mathcal{R}_{B_{1}}^{\mathrm{int}}$, and a continuous $B_{1}$-algebra isomorphism of

$$
\mathcal{R}^{\prime \prime}=\mathcal{R}_{B_{1}} \otimes_{\mathcal{R}_{B_{1}}^{\text {int }}} \mathcal{R}^{\prime}
$$

with $\mathcal{R}_{B_{1}}$ such that $M \otimes \mathcal{R}^{\prime \prime}$ is unipotent.

The proof of Theorem 5.1 .3 occupies the remainder of this chapter.

\subsection{More on unipotence}

We begin our approach to Theorem 5.1 .3 with some preliminaries. First, we widen the class of rings we are working with.

Definition 5.2.1. For $A$ a reduced affinoid algebra and $r>0$, define $R_{A, r}$ be the ring of formal series $\sum_{i \in \mathbb{Z}} a_{i} t^{i}$ over $A$ for which there exists $c>0$ such that

$$
\lim _{i \rightarrow \pm \infty} v_{A}\left(a_{i}\right)+r i-c|i|=\infty,
$$

and put

$$
w_{A, r}\left(\sum a_{i} t^{i}\right)=\min _{i}\left\{v_{A}\left(a_{i}\right)+r i\right\} .
$$

For $A$ a reduced dagger algebra, define $R_{A, r}$ as the union of $R_{B, r}$ over all fringe algebras $B$ of $A$. Define $\Omega_{R_{A, r}}^{1}$ as the free $R_{A, r}$-module generated by $d t$, with the obvious $A$-linear derivation $d: R_{A, r} \rightarrow \Omega_{R_{A, r}}^{1}$. 
Definition 5.2.2. Define a module with connection over $R_{A, r}$ as a free $R_{A, r}$-module $M$ of finite rank, equipped with an additive $A$-linear map $\nabla: M \rightarrow M \otimes \Omega_{R_{A, r}}^{1}$ satisfying the Leibniz rule. Define the operator $D: M \rightarrow M$ so that $\nabla \mathbf{v}=D \mathbf{v} \otimes \frac{d t}{t}$.

The notion of unipotent basis makes sense for modules with connection; it will help to also have a more refined notion.

Definition 5.2.3. Let $A$ be a reduced affinoid or dagger algebra. A strongly unipotent basis of a $(\sigma, \nabla)$-module over $\mathcal{R}_{A}$ relative to $A$, or a module with connection over $R_{A, r}$ for some $r>0$, is a basis $\mathbf{v}_{1}, \ldots, \mathbf{v}_{n}$ such that

$$
D \mathbf{v}_{i} \in A \mathbf{v}_{1}+\cdots+A \mathbf{v}_{i-1} \quad(i=1, \ldots, m) .
$$

Note that this definition, unlike the definition of a unipotent basis, is not stable under continuous automorphisms of $\mathcal{R}_{A}$ or $R_{A, r}$.

Lemma 5.2.4. Let $A$ be a reduced affinoid or dagger algebra. Let $M$ be a $(\sigma, \nabla)$-module over $\mathcal{R}_{A}$ relative to $A$, or a module with connection over $R_{A, r}$ for some $r>0$. Suppose $\mathbf{v}_{1}, \ldots, \mathbf{v}_{n}$ is a strongly unipotent basis of $M$. Then for $\mathbf{w} \in M, \mathbf{w}$ belongs to the $A$-span of the $\mathbf{v}_{i}$ if and only if Dw does.

Proof. One inclusion is straightforward, so suppose that $D \mathbf{w}$ is in the span of the $\mathbf{v}_{i}$. If we write $\mathbf{w}=\sum c_{i} \mathbf{v}_{i}$, then the coefficient of $\mathbf{v}_{n}$ in $D \mathbf{w}$ is precisely $t \frac{d c_{n}}{d t}$. This can only be in $A$ if it is zero. Hence $c_{n} \in A$; the same argument applied to $\mathbf{w}-c_{n} \mathbf{v}_{n}$ shows that $c_{n-1} \in A$, and so forth.

Corollary 5.2.5. Let $A$ be a reduced affinoid or dagger algebra. Let $M$ be a $(\sigma, \nabla)$-module over $\mathcal{R}_{A}$ relative to $A$, or a module with connection over $R_{A, r}$ for some $r>0$. Suppose $\mathbf{v}_{1}, \ldots, \mathbf{v}_{n}$ and $\mathbf{w}_{1}, \ldots, \mathbf{w}_{n}$ are strongly unipotent bases of $M$. Then the $A$-span of the $\mathbf{v}_{i}$ is the same as the A-span of the $\mathbf{w}_{i}$.

Proposition 5.2.6. Let $A$ be a reduced affinoid or dagger algebra. Let $M$ be a free unipotent $(\sigma, \nabla)$-module over $\mathcal{R}_{A}$ relative to $A$, or a module with connection over $R_{A, r}$ for some $r>0$. Then $M$ admits a strongly unipotent basis.

Proof. Let $\mathbf{w}_{1}, \ldots, \mathbf{w}_{n}$ be any unipotent basis of $M$. We construct the $\mathbf{v}_{i}$ so that $\mathbf{v}_{1}, \ldots, \mathbf{v}_{i}$ has the same span as $\mathbf{w}_{1}, \ldots, \mathbf{w}_{i}$ for $i=1, \ldots, n$; this will ensure that $\mathbf{v}_{1}, \ldots, \mathbf{v}_{n}$ form a basis of $M$.

To begin with, set $\mathbf{v}_{1}=\mathbf{w}_{1}$. Given $\mathbf{v}_{1}, \ldots, \mathbf{v}_{i}$, write

$$
D \mathbf{w}_{i+1}=\sum_{j=1}^{i} a_{i j} \mathbf{v}_{j} .
$$

Suppose that $a_{i j} \in A$ for $j=l+1, \ldots, i$. Write $a_{i l}=b_{i l}+\sum_{m \neq 0} c_{i l m} t^{m}$ with $b_{i l}, c_{i l m} \in A$. By Proposition 3.1.4 (or its easy analogue for $R_{A, r}$ ), we can find $e_{i l} \in \mathcal{R}_{A}$ such that $t \frac{d e_{i l}}{d t}=a_{i l}-b_{i l}$. We then observe that

$$
D\left(\mathbf{w}_{i+1}-e_{i l} \mathbf{v}_{j}\right) \in \mathcal{R}_{A} \mathbf{v}_{1}+\cdots+\mathcal{R}_{A} \mathbf{v}_{l-1}+A \mathbf{v}_{l}+\cdots+A \mathbf{v}_{i}
$$


Thus by repeating this procedure with $l=i, i-1, \ldots, 1$ in succession, we can modify $\mathbf{w}_{i+1}$, without changing the span of $\mathbf{v}_{1}, \ldots, \mathbf{v}_{i}, \mathbf{w}_{i+1}$, until we ultimately obtain a new vector which we call $\mathbf{v}_{i+1}$, with the property that

$$
D \mathbf{v}_{i+1} \in A \mathbf{v}_{1}+\cdots+A \mathbf{v}_{i}
$$

Hence this iteration produces the desired result.

We will also need one lemma from elementary number theory.

Lemma 5.2.7. Let $m, l, e$ be integers with $l, e$ positive. Then the product

$$
\prod_{i=1}^{l} \frac{m+x+i}{i}
$$

in $\mathbb{Q}_{p}[x] /\left(x^{e}\right)$ becomes an element of $\mathbb{Z}_{p}[x] /\left(x^{e}\right)$ after multiplication by $p^{a}$ for some positive integer $a \leq(e-1)\left\lceil\log _{p}(|m|+l)\right\rceil$.

Proof. The coefficient of $x^{j}$ in the product can be written as the integer $\prod_{i=1}^{l}(m+i) / i$ times a sum of products of $j$ fractions, each of the form $c /(c+m)$ for some $c \in\{1, \ldots, l\}$. From this description, the claim is evident.

\subsection{Generic unipotence}

In this section, we show that in some sense, unipotence of a module with connection over the completed fraction field implies unipotence after a localization.

Lemma 5.3.1. Let $M$ be a unipotent module with connection over $R_{K, r}$ for some $r>0$. Let $e$ be the nilpotency index of the matrix via which $D$ acts on a strongly unipotent basis of $M$. Define the functions $f_{l}: M \rightarrow M$ for $l=0,1, \ldots$ by setting $f_{0}(\mathbf{w})=D^{e-1} \mathbf{w}$ and

$$
f_{l}(\mathbf{w})=\left(1-D^{2} / l^{2}\right)^{e} f_{l-1}(\mathbf{w}) .
$$

Then for any $\mathbf{w} \in M$, the sequence $\left\{f_{l}(\mathbf{w})\right\}_{l=0}^{\infty}$ converges (under $w_{K, r}$ ) in $M$ to an element $f(\mathbf{w})$ such that $\nabla f(\mathbf{w})=0$.

Proof. Let $\mathbf{v}_{1}, \ldots, \mathbf{v}_{n}$ be a strongly nilpotent basis of $M$, and define the upper triangular nilpotent matrix $X$ over $K$ by

$$
D \mathbf{v}_{j}=\sum_{i} X_{i j} \mathbf{v}_{i}
$$

by the choice of $e, X^{e}=0$ but $X^{e-1} \neq 0$. Write $\mathbf{w}=\sum_{i} a_{i} \mathbf{v}_{i}$ and $a_{i}=\sum_{m \in \mathbb{Z}} a_{i m} t^{m}$, and put $h=\max \left\{0, \max _{i, j}\left\{-v_{K}\left(X_{i j}\right)\right\}\right\}$.

Write $f_{l}(\mathbf{w})=\sum_{i} b_{i l} \mathbf{v}_{i}$ and write $b_{i l}=\sum_{m \in \mathbb{Z}} b_{i l m} t^{m}$. We then have

$$
b_{j l m}=\sum_{i}\left((m I+X)^{e-1} \frac{\left(I-(m I+X)^{2}\right)^{e} \cdots\left(l^{2} I-(m I+X)^{2}\right)^{e}}{(l !)^{2 e}}\right)_{i j} a_{i m} .
$$


For $m=0$, this equation yields $b_{j l m}=\sum_{i}\left(X^{e-1}\right)_{i j} a_{i m}$, which does not depend on $l$. For $0<|m| \leq l$, we have $b_{j l m}=0$ because $\left(m^{2} I-(|m| I+X)^{2}\right)^{e}=0$. For $|m|>l$, we can interpret the parenthesized expression in (5.3.1.1) as the product in $\mathbb{Q}[x] /\left(X^{e}\right)$ of $(m+X)^{e-1}$ times $2 e$ expressions, each of the form $\prod_{i=1}^{l}( \pm(m+X)+i) / i$. By Lemma 5.2.7, we thus have

$$
w_{K, r}\left(b_{i(l+1)}-b_{i l}\right) \geq \min _{1 \leq i \leq n,|m|>l}\left\{v_{K}\left(a_{i m}\right)+m r-2 e(e-1)\left\lceil\log _{p}(|m|+l+1)\right\rceil-(e-1) h\right\}
$$

for $0<r \leq s$.

Choose $c, d>0$ such that $v_{K}\left(a_{i m}\right)+m r \geq c|m|-d$ for all $m$. We then have

$$
\begin{aligned}
w_{K, r}\left(b_{i(l+1)}-b_{i l}\right) & \geq \min _{x \geq l}\left\{c x-2 e(e-1) \log _{p}(x+l+1)\right\}-2 e(e-1)-d-(e-1) h \\
& \geq \min _{x \geq l}\left\{c x-2 e(e-1) \log _{p}(2 x+1)\right\}-2 e(e-1)-d-(e-1) h .
\end{aligned}
$$

The expression on the right tends to infinity with $l$; moreover, for any $0<c^{\prime}<c$, we can choose $d^{\prime}>0$ so that the right side is at least $c^{\prime} l-d^{\prime}$ for all $l$.

We conclude that $f_{l}(\mathbf{w})$ converges under $w_{K, r}$ to an element of $M$ in the $K$-span of the $\mathbf{v}_{i}$. Moreover, the limit is in the image of $D^{e-1}$ on this span, so it must be killed by one more application of $D$. This yields the desired result.

Next, we use the iteration from the previous lemma to produce horizontal elements of a $(\sigma, \nabla)$-module over a dagger algebra.

Lemma 5.3.2. Let $A$ be a reduced integral dagger algebra, and let $L$ be the completion of Frac $A$ for the affinoid topology. Let $M$ be a module with connection over $R_{A, r}$ for some $r>0$ such that $M \otimes R_{L, r}$ is unipotent. Then for any $\mathbf{w} \in M$, the element $f(\mathbf{w})$ of $M \otimes R_{L, r}$ constructed by Lemma 5.3.1 lies in $M$.

Proof. Apply Lemma 5.3.1 to $M \otimes R_{L, r}$ and retain all notation therein. Choose a basis $\mathbf{e}_{1}, \ldots, \mathbf{e}_{n}$ of $M$, and define the matrix $N$ by

$$
D \mathbf{e}_{j}=\sum_{i} N_{i j} \mathbf{e}_{j}
$$

Choose a fringe algebra $B$ over $A$ such that everything in sight defined over $R_{A, r}$ is actually defined over $R_{B, r}$ (i.e., the matrix $N$, and the coefficients of the $\mathbf{e}_{i}$ in the expansion of $\mathbf{w}$ ).

Write $f_{l}(\mathbf{w})=\sum_{i} g_{i l} \mathbf{e}_{i}$. For some $c^{\prime}, d^{\prime}>0$, we then have the estimates

$$
\begin{aligned}
& w_{L, r}\left(g_{i(l+1)}-g_{i l}\right) \geq c^{\prime} l-d^{\prime} \\
& w_{B, r}\left(g_{i(l+1)}-g_{i l}\right) \geq-2 e l\left(\frac{1}{p-1}+\max \left\{0,-w_{B, r}(N)\right\}\right)-d^{\prime} .
\end{aligned}
$$

Namely, the first estimate is obtained from the corresponding estimate for $b_{i(l+1)}-b_{i l}$ in the proof of Lemma 5.3.1 the change of basis from the $\mathbf{v}_{i}$ to the $\mathbf{e}_{i}$ only shifts the estimate by a constant. The second estimate is a straightforward consequence of the definition of $f_{l}$. 
By Proposition 2.4.2, for any rational $\epsilon \in(0,1]$, we can find a fringe algebra $C$ of $A$ containing $B$ such that

$$
|x|_{\sup , C} \leq|x|_{\text {sup }, A}^{1-\epsilon}|x|_{\text {sup }, B}^{\epsilon}
$$

for all $x \in B$. For $y \in R_{B, r}$, this implies

$$
w_{C, r}(y) \geq(1-\epsilon) w_{L, r}(y)+\epsilon w_{B, r}(y) .
$$

In particular, by taking $\epsilon$ sufficiently small, we can ensure that

$$
w_{C, r}\left(g_{i(l+1)}-g_{i l}\right) \geq c^{\prime \prime} l-d^{\prime \prime}
$$

for some $c^{\prime \prime}, d^{\prime \prime}>0$. We thus conclude that the sequences $\left\{g_{i l}\right\}_{l=0}^{\infty}$ converge under $w_{C, r}$, so their limits in $R_{L, r}$ are actually in $R_{A, r}$. (It must be checked that each limit $\sum a_{m} t^{m}$ satisfies the condition $v_{D}\left(a_{m}\right)+m r-c|m| \rightarrow \infty$ for some $c>0$ and some fringe algebra $D$, but this follows by another application of Proposition 2.4 .2 as above.) Hence $f(\mathbf{w}) \in M$, as desired.

Finally, we use what we have to obtain a result in the vein of Theorem 5.1.3,

Proposition 5.3.3. Let $A$ be a reduced integral dagger algebra, and let $L$ be the completion of Frac $A$ for the affinoid topology. Let $M$ be a module with connection over $R_{A, r}$ for some $r>0$, such that $M \otimes R_{L, r}$ is unipotent. Then $M \otimes R_{B, r}$ is unipotent for some localization $B$ of $A$.

Proof. We proceed by induction on the rank of $M$. Let $M_{0}$ be the span of a strongly unipotent basis of $M \otimes R_{L, r}$, and let $e$ be the nilpotency index of the action of $D$ on $M_{0}$. Then as w varies over $M \otimes R_{L, r}, f(\mathbf{w})$ varies over the image of $D^{e-1}$ on $M_{0}$. Since $M \otimes_{A} L$ is dense in $M \otimes R_{L, r}$, even if $\mathbf{w}$ varies only over $M \otimes_{A} L, f(\mathbf{w})$ still covers the entire image of $D^{e-1}$ on $M_{0}$. In particular, $f(\mathbf{w})$ is nonzero for some $\mathbf{w} \in M$.

Choose $\mathbf{v} \in M$ in the image of $f$ with $\mathbf{v} \neq 0$; then $\nabla \mathbf{v}=0$. Since the free submodule of $M \otimes R_{L, r}$ generated by $\mathbf{v}$ is a direct summand (because $\mathbf{v}$ belongs to the $L$-span of a strongly unipotent basis by Corollary [5.2.5), for some localization $B$ of $A$, the free submodule of $M \otimes R_{B, r}$ spanned by $\mathbf{v}$ is a direct summand. (What we are using here is that if a row vector over $R_{A, r}$ is the first row of an invertible square matrix over $R_{L, r}$, then the same is true over $R_{B, r}$ for some localization $B$ of $A$. But that is clear: any suitably close approximation of the original square matrix will still be invertible.) We may thus quotient by the submodule generated by $\mathbf{v}$ and apply the induction hypothesis to deduce the desired result.

\subsection{Generic quasi-unipotence}

We now proceed to the proof of Theorem 5.1.3. First, let us reconcile the auxiliary language in which Proposition 5.3.3 is couched with the situation of Theorem 5.1.3.

Proposition 5.4.1. Let $A$ be a reduced integral dagger algebra, and let $L$ be the completion of Frac $A$ for the affinoid topology. Let $M$ be a free $(\sigma, \nabla)$-module over $\mathcal{R}_{A}$, relative to $A$, such that $M \otimes \mathcal{R}_{L}$ is unipotent. Then $M \otimes \mathcal{R}_{B}$ is unipotent for some localization $B$ of $A$. 
Proof. Choose a basis $\mathbf{e}_{1}, \ldots, \mathbf{e}_{n}$ of $M$. Then for $r>0$ sufficiently small, the $\left(\mathcal{R}_{A} \cap R_{A, r}\right)$ span $N$ of the $\mathbf{e}_{i}$ will be a module with connection which becomes unipotent after tensoring with $R_{L, r}$. We thus conclude that $N \otimes R_{B, r}$ admits a strongly unipotent basis $\mathbf{v}_{1}, \ldots, \mathbf{v}_{n}$ for some localization $B$ of $A$. On the other hand, $N \otimes\left(\mathcal{R}_{L} \cap R_{L, r}\right)$ also admits a strongly unipotent basis; by Corollary 5.2.5, these two bases have the same $L$-span within $N \otimes R_{L, r}$. Consequently the $\mathbf{v}_{i}$ form a basis of

$$
\begin{aligned}
\left(N \otimes \mathcal{R}_{L}\right) \cap\left(N \otimes R_{B, r}\right) & =N \otimes\left(\mathcal{R}_{L} \cap R_{B, r}\right) \\
& =N \otimes\left(\mathcal{R}_{B} \cap R_{B, r}\right) .
\end{aligned}
$$

This yields the desired result.

Remark 5.4.2. In some cases, it may be possible to show that the conclusion of Proposition 5.4 .1 holds without the localization. For instance, this can be shown in case $M$ is a "log- $(\sigma, \nabla)$-module" over $\mathcal{R}^{+}$(in which the module of differentials is enlarged to include $d t / t)$; this is a variant of "Dwork's trick" dJ3, Lemma 6.3].

Theorem 5.1.3 can now be deduced as follows.

Proof of Theorem 5.1.3. We will treat only the case where $A$ is a dagger algebra; for the affinoid case, the argument is the same except that any fringe algebra of $A$ should be replaced by $A$ itself.

Let $L$ be the completion of Frac $A$ for the affinoid topology. Apply Theorem 5.1 .2 to obtain an integer $m \geq 0$, a finite unramified extension $L_{1}$ of $L_{0}=L^{\sigma^{-m}}$, a finite étale extension $\mathcal{R}_{L}^{\prime}$ of $\mathcal{R}_{L_{1}}^{\text {int }}$, and a continuous $L_{1}$-algebra isomorphism of

$$
\mathcal{R}_{L}^{\prime \prime}=\mathcal{R}_{L_{1}} \otimes_{\mathcal{R}_{L_{1}}^{\text {int }}} \mathcal{R}_{L}^{\prime}
$$

with $\mathcal{R}_{L_{1}}$ such that $M \otimes \mathcal{R}_{L}^{\prime \prime}$ is unipotent. Recall that $M \otimes \mathcal{R}_{L}^{\prime \prime}$ is actually unipotent for any choice of the last isomorphism.

Let $X$ be the special fibre of $A_{0}=A^{\sigma^{-m}}$, and let $Y$ be the normalization of $X$ in the residue field of $L_{1}$. Since $L_{1} / L_{0}$ is unramified, its residue field extension is separable: that is, the natural finite morphism $f: Y \rightarrow X$ is generically étale. The residue field of $\mathcal{R}_{L_{1}}^{\text {int }}$ can be canonically identified with $K(Y)((t))$, where $K(Y)$ is the function field of $Y$ (i.e., the residue field of $\left.L_{1}\right)$; the residue field of $\mathcal{R}_{L}^{\prime}$ is a finite separable extension of $K(Y)((t))$.

We can choose an open dense subset $X^{\prime}$ of $X$ such that the following conditions hold.

(a) The restriction of $f$ to $Y^{\prime}=f^{-1}\left(X^{\prime}\right)$ is finite étale.

(b) For $R$ the coordinate ring of $Y^{\prime}$, the integral closure of $R[[t]]$ in the residue field of $\mathcal{R}_{L}^{\prime}$ is isomorphic over $R$ to (another copy of) $R[[t]]$.

(c) The complement $X \backslash X^{\prime}$ is the zero locus of some regular function on $X$. 
Choose a localization $A_{0}^{\prime}$ of $A_{0}$ with special fibre $X^{\prime}$, and let $A_{1}^{\prime}$ be the integral closure of $A_{0}^{\prime}$ in $L_{1}$. Then $A_{1}^{\prime}$ is a dagger algebra of MW-type with special fibre $Y^{\prime}$, and the isomorphism in (b) lifts (noncanonically) to an isomorphism of the integral closure of $\mathcal{R}_{A_{1}^{\prime}}$ in $\mathcal{R}_{L}^{\prime \prime}$ with $\mathcal{R}_{A_{1}^{\prime}}$.

We may thus apply Proposition 5.4.1 to deduce that $M$ becomes unipotent after tensoring with the integral closure of $\mathcal{R}_{A_{1}^{\prime \prime}}$ in $\mathcal{R}_{L}^{\prime \prime}$, for some localization $A_{1}^{\prime \prime}$ of $A_{1}^{\prime}$. Choose a localization $B_{0}$ of $A_{0}^{\prime}$ whoes integral closure $B_{1}$ in $L_{1}$ contains $A_{1}^{\prime \prime}$, and put $B=B_{0}^{\sigma^{m}}$. Let $\mathcal{R}^{\prime \prime}$ denote the integral closure of $\mathcal{R}_{B_{1}}$ in $\mathcal{R}^{\prime \prime}$; we now have a continuous $B_{1}$-linear isomorphism of $\mathcal{R}^{\prime \prime}$ with $\mathcal{R}_{B_{1}}$, under which $M \otimes \mathcal{R}^{\prime \prime}$ becomes unipotent. This is the desired result.

\section{Cohomology of curves}

In this chapter, we establish the finite dimensionality of the cohomology of an overconvergent $F$-isocrystal on an affine curve. Strictly speaking, this chapter can be skipped, as there are several other techniques available for establishing this fact (see Remark 6.4.3). However, we decided to include this argument because it is most in the spirit of the rest of the paper (in particular, it can be used in the relative setting, although we will not do so), and because its strategy may be of use for computational purposes.

\subsection{Near-isomorphisms}

Given a short exact sequence

$$
0 \rightarrow A \rightarrow B \rightarrow C \rightarrow 0
$$

of $K$-vector spaces, $B$ is finite dimensional if and only if $A$ and $C$ both are. Thus within the abelian category of $K$-vector spaces, the full subcategory of finite dimensional vector spaces is a Serre subcategory, and we can form the quotient category.

Definition 6.1.1. We will denote properties of morphisms that hold in the quotient category by the prefix "near". That is, a $K$-linear transformation $f: A \rightarrow B$ is a near-injection if $\operatorname{ker}(f)$ is finite dimensional, a near-surjection if coker $(f)$ is finite dimensional, and a nearisomorphism if it is both near-injective and near-surjective.

From the Serre construction, the following facts are evident:

- If $f: A \rightarrow B$ and $g: B \rightarrow C$ are $K$-linear transformations such that $g \circ f$ is a near-injection (resp. is a near-surjection), then so is $f$ (resp. so is $g$ ).

- If $f: A \rightarrow B$ and $g: B \rightarrow C$ are $K$-linear transformations and any two of $f, g, g \circ f$ are near-isomorphisms, then so is the third.

- Let

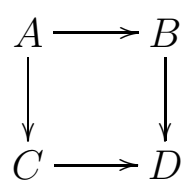


be a commuting square of $K$-linear transformations. If any three of the maps are near-isomorphisms, then so is the fourth.

\subsection{Constant coefficients}

Hypothesis 6.2.1. Throughout this section unless otherwise specified, let $A$ be a dagger algebra of MW-type whose special fibre $X=\operatorname{Spec} R$ is a (smooth affine) irreducible curve over $k$ such that, for $\bar{X}$ a smooth compactification of $X$, the points of the reduced scheme $\bar{X} \backslash X$ are geometrically reduced. (This is automatic if $k$ is perfect.)

In this section, we show that the cohomology of $A$, as a $(\sigma, \nabla)$-module over itself, is finite dimensional. This result was first shown by Monsky [Mo] our argument is a form of that argument recast in a setting due to Crew $[\mathrm{Cr}$, which will pave the way for an argument involving coefficients. (We will return to this setting in Chapter [7.)

First recall a consequence of [MW, Theorem 6.2].

Lemma 6.2.2. Any finitely generated $A$-module which is annihilated by some element of $A$ is finite dimensional over $K$. In particular, any homomorphism of finitely generated $A$-modules which becomes an isomorphism over $\operatorname{Frac}(A)$ is a near-isomorphism of K-vector spaces.

This lemma can also be proved by reducing to the case $A=K\langle t\rangle^{\dagger}$ by Noether normalization, then using Weierstrass preparation to show that any ideal of $K\langle t\rangle^{\dagger}$ is generated by a polynomial in $t$.

Following Crew [Cr, 7.2-7.3], we associate to each point of $\bar{X} \backslash X$ a "local algebra" isomorphic to a Robba ring. First (again following Crew) we do this for points on $X$. Given $x \in X$ with residue field $k^{\prime}$, let $\mathfrak{m}_{x}$ be the preimage in $A^{\text {int }}$ of the maximal ideal of $R$ at $x$. Then by the Cohen structure theorem, the $\mathfrak{m}_{x}$-adic completion of $A^{\text {int }}$ is homeomorphic to $\mathcal{O}^{\prime} \llbracket t \rrbracket=\mathcal{R}_{K^{\prime}}^{+ \text {,int }}$, for $K^{\prime}$ the unramified extension of $k$ with residue field $k^{\prime}$ and $\mathcal{O}^{\prime}$ the ring of integers of $K^{\prime}$.

Now we consider the missing points.

Definition 6.2.3. Let $x_{1}, \ldots, x_{m}$ be the (closed) points of $\bar{X} \backslash X$, and let $K_{i}$ be the unramified extension of $K$ whose residue field $k_{i}$ is the residue field of $x_{i}$. Choose an open affine $U$ of $\bar{X}$ containing all of the $x_{i}$, and let $B$ be a dagger algebra with special fibre $U$. Let $\mathcal{R}_{i}$ be a copy of the Robba ring with residue field $K_{i}$ and series parameter $t_{i}$, and choose an embedding $B^{\text {int }} \hookrightarrow \mathcal{R}_{i}^{+ \text {,int }}$ as in the previous paragraph. This extends to an embedding $C^{\text {int }} \hookrightarrow \mathcal{R}_{i}^{\text {int }}$, for $C$ the localization of $B$ with special fibre $U-\left\{x_{1}, \ldots, x_{m}\right\}$. Choose an embedding of $A$ into $C$, and let $\rho_{i}: A^{\text {int }} \rightarrow \mathcal{R}_{i}^{\text {int }}$ be the resulting embedding; note that any Frobenius lift on $A$ induces a compatible Frobenius lift on $C$, hence also on $\mathcal{R}_{i}$. Let $\rho: A^{\text {int }} \rightarrow \oplus_{i} \mathcal{R}_{i}^{\text {int }}$ denote the direct sum of the $\rho_{i}$.

Remark 6.2.4. We mention in passing Crew's description of the above construction, which is more geometric. Choose a formally smooth lifting of $\bar{X}$, and let $\mathcal{R}_{i}$ be the union, over strict neighborhoods $V$ of the tube of $X$, of the ring of rigid analytic functions on the intersection of $V$ with the tube of $x_{i}$. 
The embedding $\rho$ is a "faithful" description of the dagger algebra $A$ in the following sense. (For a much stronger result, see [Cr, Theorem 7.5].)

Proposition 6.2.5. The image of $\rho$ is closed for the $\pi$-adic topology.

The proposition is a consequence of the following lemma together with the weak completeness of $A$.

Lemma 6.2.6. Let $R=k\left[y_{1}, \ldots, y_{n}\right] / \mathfrak{a}$ be the coordinate ring of a smooth affine curve $X$ over $k$, and let $\bar{X}$ be a smooth compactification of $X$. For $r \in R$, let $d(r)$ denote the maximum pole degree of $r$ at any point of $\bar{X} \backslash X$. Then for some $c>0$, every $r \in R$ can be lifted to an element of $k\left[y_{1}, \ldots, y_{n}\right]$ of degree at most $c d(r)$.

Proof. It is enough to produce a single set of generators of $R$ for which the result holds. Let $x_{1}, \ldots, x_{m}$ be the points of $\bar{X} \backslash X$, and for $r \in R$, let $p(r)$ be the $m$-tuple whose $i$-th component is $\max \left\{0,-\operatorname{deg}_{x_{i}}(r)\right\}$. By Riemann-Roch, $p(r)$ is the zero tuple if and only if $r$ is in the integral closure of $k$.

The submonoid of $\mathbb{Z}_{>0}^{m}$ generated by the image of $p$ is finitely generated (again by Riemann-Roch, which implies that the submonoid contains all but finitely elements of $\mathbb{Z}_{\geq 0}^{m}$ ); choose $z_{1}, \ldots, z_{l} \in R$ whose images under $p$ generate this monoid. Then there is a straightforward division algorithm to write $r \in R$ in terms of the $z_{i}$ : manufacture a product of $z_{j}$ 's with the same leading term as $r$ in the pole expansion at each $x_{i}$, subtract off this monomial, and repeat. This gives the desired estimate.

Remark 6.2.7. Note that the images of the individual $\rho_{i}$ need not be closed if $m>1$; for instance, consider the example $A=K\left\langle x, x^{-1}\right\rangle^{\dagger}$ with embeddings into $\mathcal{R}_{K}$ given by $x \mapsto t$ and $x \mapsto t^{-1}$. On the other hand, if we $p$-adically complete $A^{\text {int }}$ and $\mathcal{R}_{i}^{\text {int }}$, then the image of the map induced by $\rho_{i}$ is closed.

By Riemann-Roch, the maps

$$
R \rightarrow \oplus_{i}\left(k_{i}\left(\left(\bar{t}_{i}\right)\right) / k_{i} \llbracket \bar{t}_{i} \rrbracket\right), \quad \Omega_{R / k}^{1} \rightarrow \oplus_{i}\left(\Omega_{\left.k_{i}\left(\bar{t}_{i}\right)\right) / k_{i}}^{1} / \Omega_{k_{i} \llbracket \bar{t}_{i} \rrbracket / k_{i}}^{1}\right)
$$

induced by the reduction of $\rho$ are near-isomorphisms of $k$-vector spaces. Our next lemmas extend this property to $\rho$ itself.

Lemma 6.2.8. For any $a, b \in A$ with $b \neq 0$, the map

$$
\rho: \frac{a}{b} A \rightarrow \oplus_{i} \mathcal{R}_{i} / \mathcal{R}_{i}^{+}
$$

is a near-isomorphism of $K$-vector spaces. In particular, we can choose a so that the map is injective when $b=1$, and we can choose $b$ so that the map is surjective when $a=1$.

Proof. The near-injectivity and near-surjectivity for any given $a, b$ are equivalent to the same statements for $a, b=1$, because the inclusions $a A \hookrightarrow(a / b) A$ and $a A \hookrightarrow A$ are nearisomorphisms by Lemma 6.2.2 It thus suffices to prove the claims of the final sentence. 
The injectivity is easy: for any $a \in A^{\text {int }}$ whose reduction has a pole at each $x_{i}$, the reduction of the map $\rho: a A^{\text {int }} \rightarrow \oplus_{i} \mathcal{R}_{i}^{\text {int }} / \mathcal{R}_{i}^{+ \text {,int }}$ is injective by Riemann-Roch. Thus $\rho$ injects $a A$ into $\oplus_{i} \mathcal{R}_{i} / \mathcal{R}_{i}^{+}$.

As for surjectivity, again by Riemann-Roch we can choose $b \in A^{\text {int }}$ with nonzero reduction so that the reduction of $\rho: \frac{1}{b} A^{\text {int }} \rightarrow \oplus_{i} \mathcal{R}_{i}^{\text {int }} / \mathcal{R}_{i}^{+ \text {,int }}$ is surjective. Thus the image of $\frac{1}{b} A$ in $\oplus_{i} \mathcal{R}_{i} / \mathcal{R}_{i}^{+}$is dense; since the image is also closed by Proposition 6.2.5, it is full.

Lemma 6.2.9. The map

$$
d \rho: \Omega_{A / K}^{1} \rightarrow \oplus_{i} \Omega_{\mathcal{R}_{i} / K}^{1} / \Omega_{\mathcal{R}_{i}^{+} / K}^{1}
$$

is a near-isomorphism of $K$-vector spaces.

Proof. Again, the near-injectivity follows from the corresponding statement on reductions. As for near-surjectivity, let $u$ be any element of $A$ which is not integral over $K$. By Lemma 6.2.8, we can choose $w \in A$ such that $\frac{1}{w} A \rightarrow \oplus_{i} \mathcal{R}_{i} / \mathcal{R}_{i}^{+}$is surjective; we can thus find $v \in A$ such that $v / w$ maps to the class of $d t_{i} / d u$ in $\mathcal{R}_{i} / \mathcal{R}_{i}^{+}$for all $i$. This means the map

$$
\frac{v}{w} \Omega_{A / K}^{1} \rightarrow \oplus_{i} \Omega_{\mathcal{R}_{i} / K}^{1} / \Omega_{\mathcal{R}_{i}^{+} / K}^{1}
$$

induced by $d \rho$ is surjective. As in Lemma 6.2.8 this means that $d \rho$ is near-surjective, as desired.

Proposition 6.2.10. Let $A$ be a dagger algebra of $M W$-type whose special fibre $X$ is onedimensional over $k$. Then $d: A \rightarrow \Omega_{A / K}^{1}$ is a near-isomorphism, that is, $H_{\mathrm{rig}}^{0}(X / K)$ and $H_{\mathrm{rig}}^{1}(X / K)$ are finite dimensional.

In particular, in the statement of the proposition, we do not assume that $X$ is irreducible or that $\bar{X} \backslash X$ is geometrically reduced. However, we may make these assumptions without loss of generality, the former because formation of $\Omega^{1}$ commutes with direct sums, the latter because we can replace $k$ by a finite extension thanks to Proposition 3.4.3. Thus we may continue to assume Hypothesis 6.2.1.

Proof. By Lemma 6.2.8 and Lemma 6.2.9, the horizontal arrows in the diagram

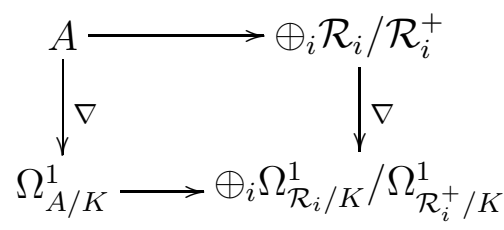

are near-isomorphisms. The right vertical arrow is also a near-isomorphism: in each factor, the kernel is trivial and the cokernel is generated by $d t_{i} / t_{i}$. Since three of the arrows are near-isomorphisms, the fourth must be as well.

In fact, with a bit more care, one can extract from this argument that $H_{\text {rig }}^{1}(X / K)$ has the expected dimension, namely twice the genus of a smooth compactification $\bar{X}$, plus the degree of $\bar{X} \backslash X$, minus one. 


\subsection{Interlude: matrix factorizations}

To handle general $(\sigma, \nabla)$-modules, we will need a factorization lemma in the vein of those from [Ke1, Chapter 6], but which accounts for the embedding $\rho$. We develop that lemma in this section.

First recall the statement of [Ke1, Proposition 6.5].

Lemma 6.3.1. Let $U$ be an invertible $n \times n$ matrix over $\mathcal{R}_{K}$. Then $U$ can be written as a product $V W$, with $V$ an invertible matrix over $\mathcal{R}_{K}^{\text {int }}\left[\frac{1}{p}\right]$ and $W$ an invertible matrix over $\mathcal{R}_{K}^{+}$.

With a bit more care, we can shift the denominators from $V$ to $W$. First, we recall some terminology from linear algebra.

Definition 6.3.2. Recall that an elementary matrix over a ring $R$ is defined as a matrix over $R$ obtained from the identity matrix by performing one of the following operations:

(a) multiplying a row by a unit in $R$;

(b) switching two rows;

(c) adding a multiple of one row to another.

Multiplying a matrix $U$ by an elementary matrix on the right effects the corresponding operation on $U$.

Lemma 6.3.3. Let $U$ be an invertible $n \times n$ matrix over $\mathcal{R}_{K}$. Then $U$ can be written as a product $V W$, with $V$ an invertible matrix over $\mathcal{R}_{K}^{\text {int }}$ and $W$ an invertible matrix over $\mathcal{R}_{K}^{+}$.

Proof. By Lemma 6.3.1, we may reduce to the case where $U$ itself is invertible over $\mathcal{R}_{K}^{\operatorname{int}}\left[\frac{1}{p}\right]$. Namely, a general $U$ admits the decomposition $V_{1} W_{1}$ from Lemma 6.3.1. If we can then decompose $V_{1}=V_{2} W_{2}$ with $V_{2}$ invertible over $\mathcal{R}_{K}^{\text {int }}$ and $W_{2}$ invertible over $\mathcal{R}_{K}^{+}$, we may take $V=V_{2}$ and $W=W_{2} W_{1}$ and we are done.

We thus assume that $U$ is invertible over $\mathcal{R}_{K}^{\text {int }}\left[\frac{1}{p}\right]$. We may also assume that $U$ has integral entries, though its inverse may not.

We induct on $v_{K}(\operatorname{det}(U))$; if it is zero, then $U^{-1}$ has integral entries and we are done. Otherwise, the reduction of $U$ is a matrix over $k((t))$ with less than full rank. By the usual theory of elementary divisors over $k \llbracket t \rrbracket$, we can find a sequence of elementary row operations on the reduction which will produce a zero row. Namely, perform the Euclidean algorithm on entries in the first column until at most one is nonzero; if there remains a nonzero entry, strike out that row. Then perform the Euclidean algorithm on entries in the second column in the remaining rows, and so on.

The coefficients of the corresponding elementary matrices can be lifted to $\mathcal{O} \llbracket t \rrbracket$. The product of these lifts, together with an elementary matrix that divides the offending row by $\pi$, yields an invertible matrix over $\mathcal{R}_{K}^{+}$whose product on the right with $U$ has determinant of strictly smaller valuation than that of $U$. Thus the induction hypothesis can be used to complete the decomposition, yielding the desired result. 
We now introduce Hypothesis 6.2.1 and give a lemma of a similar flavor.

Lemma 6.3.4. For any sequence $U_{1}, \ldots, U_{m}$ with $U_{i}$ an invertible $n \times n$ matrix over $\mathcal{R}_{i}$, there exists a matrix $V$ over Frac $A$ such that $U_{i} \rho_{i}(V)$ is an invertible matrix over $\mathcal{R}_{i}^{+}$for $i=1, \ldots, n$.

Proof. By Lemma 6.3.3, we can find an invertible matrix $Q_{i}$ over $\mathcal{R}_{i}^{+}$such that $Q_{i} U_{i}$ is invertible over $\mathcal{R}_{i}^{\text {int }}$. By Riemann-Roch, we can simultaneously approximate the reductions of the $Q_{i} U_{i}$ arbitrarily well by the reductions of matrices over $(\operatorname{Frac} A)^{\text {int }}$. In particular, we can choose an invertible matrix $P$ over $(\text { Frac } A)^{\text {int }}$ such that for some $r>0, w_{l}\left(Q_{i} U_{i} \rho_{i}(P)\right)>0$ for $0<l \leq r$ and $i=1, \ldots, m$. We can then find an invertible matrix $R_{i}$ over $\mathcal{R}_{i}^{+ \text {,int }}$ such that $v_{K}\left(R_{i} Q_{i} U_{i} \rho_{i}(P)-I\right)>0$. For simplicity, put $U_{i}^{\prime}=R_{i} Q_{i} U_{i} \rho_{i}(P)$ and put $h=$ $\min _{i}\left\{v_{K_{i}}\left(U_{i}^{\prime}-I\right)\right\}>0$.

By Lemma 6.2.8, we can find $b \in A^{\text {int }}$ such that $\rho: b^{-1} A^{\text {int }} \rightarrow \oplus_{i} \mathcal{R}_{i}^{\text {int }} / \mathcal{R}_{i}^{+ \text {,int }}$ is surjective. We construct sequences $\left\{V_{j}\right\}_{j=1}^{\infty},\left\{W_{i, j}\right\}_{j=1}^{\infty}$ of matrices, where $V_{j}$ has entries in $b^{-1} A^{\text {int }}$ and $W_{i, j}$ has entries in $\mathcal{R}_{i}^{+, \text {int }}$, such that

$$
v_{K}\left(U_{i}^{\prime} \rho_{i}\left(V_{j}\right)-W_{i, j}\right) \geq j h .
$$

Namely, we start with $V_{1}=W_{i, 1}=I$. Given $V_{j}$ and $W_{i, j}$ for some $j$, write $U_{i}^{\prime} \rho_{i}\left(V_{j}\right)-W_{i, j}=$ $X_{i, j}$. Then choose matrices $Y_{j}$ over $\pi^{j} b^{-1} A^{\text {int }}$ and $Z_{i, j}$ over $\mathcal{R}_{i}^{+ \text {,int }}$ so that $X_{i, j}=\rho_{i}\left(Y_{j}\right)+Z_{i, j}$, $v_{K}\left(Y_{j}\right) \geq v_{K_{i}}\left(X_{i, j}\right)$, and $v_{K_{i}}\left(Z_{i, j}\right) \geq v_{K_{i}}\left(X_{i, j}\right)$. Finally, set $V_{j+1}=V_{j}-Y_{j}$ and $W_{i, j+1}=$ $W_{i, j}+Z_{i, j}$. Then

$$
\begin{aligned}
v_{K_{i}}\left(U_{i}^{\prime} \rho_{i}\left(V_{j+1}\right)-W_{i, j+1}\right) & =v_{K_{i}}\left(U_{i}^{\prime} \rho_{i}\left(V_{j}-Y_{j}\right)-W_{i, j}-Z_{i, j}\right) \\
& =v_{K_{i}}\left(\left(I-U_{i}^{\prime}\right) \rho\left(Y_{j}\right)\right) \\
& \geq(j+1) h,
\end{aligned}
$$

and the iteration continues.

From the construction, we see that $v_{K_{i}}\left(W_{i, j+1}-W_{i, j}\right) \geq j h$. Consequently each sequence $\left\{W_{i, j}\right\}_{j=1}^{\infty}$ converges to an invertible matrix $W_{i}$ over $\mathcal{R}_{i}^{+, \text {int }}$. The entries in the direct sum of the matrices $W_{i}^{-1} U_{i}^{\prime}$ are in the closure of the image of $\rho$ on $b^{-1} A$. By Proposition 6.2.5, there is a matrix $V^{\prime}$ over $b^{-1} A$ with $U_{i}^{\prime} \rho_{i}\left(V^{\prime}\right)=W_{i}$ for all $i$. We may thus take $V=P V^{\prime}$.

\subsection{General coefficients}

We now analyze the cohomology of $(\sigma, \nabla)$-modules on a curve, focusing on the crucial case of unipotent local monodromy.

Proposition 6.4.1. Let $M$ be a $(\sigma, \nabla)$-module over $A$. Assume that $M \otimes \mathcal{R}_{i}$ is unipotent for each $i$. Then there is a commuting diagram

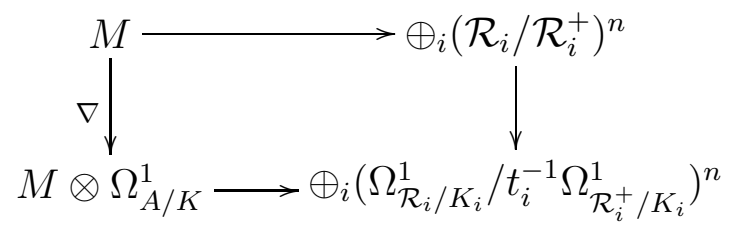


in which the horizontal arrows and the right vertical arrow are near-isomorphisms of $K$ vector spaces. Consequently, $\nabla: M \rightarrow M \otimes \Omega_{A / K}^{1}$ is a near-isomorphism.

Proof. Since $M$ is locally free over $A$, we can choose a basis $\mathbf{e}_{1}, \ldots, \mathbf{e}_{n}$ of $M$ over $A\left[a^{-1}\right]$ for some $a$. (Note that $A\left[a^{-1}\right]$ is the localization in the category of rings, not dagger algebras.) Define the matrices $N_{i}$ over $\mathcal{R}_{i}$ by $\nabla \mathbf{e}_{l}=\sum_{j}\left(N_{i}\right)_{j l} \mathbf{e}_{j} \otimes \frac{d t_{i}}{t_{i}}$. By the unipotence hypothesis and Proposition 5.2.6, there exist matrices $U_{i}$ over $\mathcal{R}_{i}$ such that $X_{i}=U_{i}^{-1} N_{i} U_{i}+U_{i}^{-1} t_{i} \frac{d}{d t_{i}} U_{i}$ is a nilpotent matrix over $K_{i}$.

By Lemma 6.3.4 there exists a matrix $V$ over Frac $A$ such that $W_{i}=U_{i}^{-1} \rho_{i}(V)$ and its inverse have entries in $\mathcal{R}_{i}^{+}$for $i=1, \ldots, m$. Put $\mathbf{v}_{l}=\sum_{j} U_{j l} \mathbf{e}_{j}$ for $l=1, \ldots, n$. We now can describe the horizontal arrows in (6.4.1.1) as follows. Form the free module $S$ over $\oplus_{i} \mathcal{R}_{i}$ generated by $\mathbf{w}_{1}, \ldots, \mathbf{w}_{n}$, map $M$ to this module by sending $\sum_{l} c_{l} \mathbf{v}_{l}$ to $\sum_{l} \rho\left(c_{l}\right) \mathbf{w}_{l}$, and quotient as needed.

Put

$$
N_{i}^{\prime}=\rho(V)^{-1} N_{i} \rho(V)+\rho(V)^{-1} t_{i} \frac{d}{d t_{i}} \rho(V)=W_{i}^{-1} X_{i} W_{i}+W_{i}^{-1} t \frac{d}{d t} W_{i},
$$

which has entries in $\mathcal{R}^{+}$. Then the right vertical arrow in (6.4.1.1) is induced by the map $S \rightarrow S \otimes \oplus_{i} \Omega_{\mathcal{R}_{i} / K_{i}}^{1}$ given by

$$
\sum_{l} c_{l} \mathbf{w}_{l} \mapsto \sum_{l} \mathbf{w}_{l} \otimes d c_{l}+\sum_{j, l}\left(N_{i}^{\prime}\right)_{j l} c_{l} \mathbf{w}_{j} \otimes \frac{d t}{t}
$$

The right vertical arrow is actually an isomorphism, by a straightforward calculation (or skip ahead to Proposition (7.1.3). The top horizontal arrow can be factored as an inclusion $M \rightarrow \frac{1}{u} A^{n}$ (for some $u \in A$ ) followed by $\rho$, which are near-isomorphisms by Lemma 6.2.2 and Lemma 6.2.8, respectively; hence it is a near-isomorphism. Similarly, the bottom horizontal arrow is a near-isomorphism by Lemma 6.2.2 and Lemma 6.2.8.

Thus three of the arrows in (6.4.1.1) are near-isomorphisms, so the left vertical arrow is as well.

Theorem 6.4.2. Let $X$ be a smooth affine curve over $k$ and let $\mathcal{E}$ be an overconvergent $F$-isocrystal on $X$. Then $H^{i}(X / K, \mathcal{E})$ is finite dimensional over $K$ for all $i$.

Proof. Let $A$ be a dagger algebra of MW-type with special fibre $X$, let $\sigma: A \rightarrow A$ be a Frobenius lift, and let $M$ be a $(\sigma, \nabla)$-module on $A$ corresponding to $\mathcal{E}$. As in Proposition 6.2.10, by pulling back along a suitable cover, we may reduce to the case where Hypothesis 6.2.1 holds; we may also reduce to the case where $M \otimes \mathcal{R}_{i}$ is unipotent for each $i$. But then Proposition 6.4.1 asserts that $H^{0}(M)$ and $H^{1}(M)$ are finite dimensional over $K$. This yields the desired result.

Remark 6.4.3. As mentioned earlier, we know of three other ways to prove Theorem 6.4.2 (at least for $k$ perfect, but one can easily reduce to this case). One proof is given by Crew $[\mathrm{Cr}$, who establishes finiteness of cohomology on a curve using techniques of $p$-adic functional analysis (the key argument being that a locally convex space which is both Fréchet 
and dual-of-Fréchet is finite dimensional). A second approach is to "compactify coefficients" as in Ke2 and then apply Kiehl's finiteness theorems [Ki], or put another way, invoke a comparison between rigid and log-crystalline cohomology. A third approach, closely related to the second, is to "algebrize" the connection and compare rigid cohomology to algebraic de Rham cohomology [CM2, Corollaire 5.0-12].

Remark 6.4.4. As noted at the beginning of this chapter, one can actually deduce the existence of generic pushforwards by relativizing the proof of Theorem 6.4.2. However, the argument is somewhat messier than the one we use in the next chapter, and gives less precise information. We thus leave it as an exercise for the interested reader.

\section{Pushforwards in rigid cohomology}

In this section, we establish the existence of "generic" pushforwards, with and without supports, for an overconvergent $F$-isocrystal on a family of affine curves. A similar result for families of projective curves has been given by Tsuzuki [T6].

\subsection{Relative local cohomology}

The main relevance of a relative version of the $p$-adic local monodromy theorem in this paper is that it controls the local cohomology of a $(\sigma, \nabla)$-module over the Robba ring over a dagger algebra. In this section, we illustrate how this control is exerted, starting with a computation in the unipotent case.

Lemma 7.1.1. Let $A$ be a reduced dagger algebra equipped with a Frobenius lift. Let $M$ be a free unipotent $(\sigma, \nabla)$-module over $\mathcal{R}_{A}$, relative to $A$. Then $H^{0}(M)$ and $H^{1}(M)$ are finitely generated $A$-modules.

Proof. By Proposition 5.2.6, there exists a strongly unipotent basis $\mathbf{v}_{1}, \ldots, \mathbf{v}_{n}$ of $M$. In terms of this basis, it is easy to read off the kernel and cokernel of $\nabla$.

- Any element of the kernel of $\nabla$ must lie in the $A$-span of the $\mathbf{v}_{i}$. Hence $\operatorname{ker}(\nabla)=H^{0}(M)$ is a submodule of a finitely generated $A$-module, and so is finitely generated.

- Any element of $M \otimes \Omega_{\mathcal{R}_{A} / A}^{1}$ can be formally written as

$$
\sum_{i=1}^{n} \sum_{l \in \mathbb{Z}} f_{i l} t^{l} \mathbf{v}_{i} \otimes \frac{d t}{t}
$$

for some $f_{i l} \in A$. If $f_{i 0}=0$ for $i=1, \ldots, n$, then this element is in the image of $\nabla$. Hence coker $(\nabla)=H^{1}(M)$ is a quotient of a finitely generated $A$-module, and so is finitely generated. 
Remark 7.1.2. From the proof, we can easily read off two topological facts about a unipotent $(\sigma, \nabla)$-module $M$ over $\mathcal{R}_{A}$, for $A$ a reduced dagger algebra.

(a) The image of $\nabla$ is closed.

(b) The continuous bijection $M / \operatorname{ker}(\nabla) \rightarrow \operatorname{im}(\nabla)$ is a homeomorphism.

These will later form the basis for more general statements of the same form.

In the manner of Lemma 7.1.1 we can also verify the observation that was made without proof in the course of proving Theorem 6.4.2.

Proposition 7.1.3. Let $M$ be a unipotent $(\sigma, \nabla)$-module over $\mathcal{R}_{K}$. Let $\mathbf{v}_{1}, \ldots, \mathbf{v}_{n}$ be a strongly unipotent basis of $M$, and let $M_{1}$ be the $\mathcal{R}_{K}^{+}$-span of the $\mathbf{v}_{i}$. Then the induced map

$$
\nabla: M / M_{1} \rightarrow(M \otimes d t) /\left(M_{1} \otimes \frac{d t}{t}\right)
$$

is an isomorphism.

Proof. The fact that the map is an isomorphism means two things, both of which are evident from the special form of $\nabla$.

- If $\mathbf{v} \in M$ satisfies $\nabla \mathbf{v} \in M_{1} \otimes \frac{d t}{t}$, then $\mathbf{v} \in M_{1}$. This follows because we may assume the coefficient of $t^{j}$ in $\mathbf{v}$ is zero for $j \geq 0$, but then any power of $t$ which appears in $\mathbf{v}$ also appears in $\nabla \mathbf{v}$.

- For every element $\omega$ of $M \otimes \frac{d t}{t}$, there exists $\mathbf{v} \in M$ such that $\nabla \mathbf{v}-\omega \in M_{1} \otimes d t / t$. This follows because once we subtract off the coefficient of $d t / t$ in $\omega$, the result maps to zero in the cokernel of $\nabla: M \rightarrow M \otimes d t$ as seen in the proof of Lemma [7.1.1]

Now we consider relative local cohomology in the non-unipotent case.

Proposition 7.1.4. Let $A$ be an integral dagger algebra of $M W$-type equipped with a Frobenius lift. Let $M$ be a free $(\sigma, \nabla)$-module over $\mathcal{R}_{A}$, relative to $A$, and let $B$ be a localization satisfying the conclusion of Theorem 5.1.3. Then $H^{0}\left(M \otimes \mathcal{R}_{B}\right)$ and $H^{1}\left(M \otimes \mathcal{R}_{B}\right)$ are finitely generated $B$-modules.

Proof. An extension $\mathcal{R}^{\prime \prime}$ as in Theorem [5.1.3 is the composition of two extensions as in Remark 3.2.7 (the first because $B^{\sigma^{-m}}$ is flat over $B$ by Remark 3.2.4), so $H^{i}\left(M \otimes \mathcal{R}_{B}\right)$ injects into $H^{i}\left(M \otimes \mathcal{R}^{\prime \prime}\right)$ for $i=0,1$. Since $H^{i}\left(M \otimes \mathcal{R}^{\prime \prime}\right)$ is finitely generated by Lemma 7.1.1, we have the desired result.

Remark 7.1.5. Over $K$, one can also deduce Proposition 7.1.4 from the $p$-adic index theorem of Christol-Mebkhout CM1, Théorème 7.5-2]. We do not know whether that theorem admits a relative version from which one can deduce Proposition 7.1 .4 in general. 


\subsection{Base change in local cohomology}

We next examine how the local pushforwards we have constructed behave with respect to flat base change.

Proposition 7.2.1. Let $A$ be an integral dagger algebra of $M W$-type equipped with a Frobenius lift. Let $M$ be a free $(\sigma, \nabla)$-module over $\mathcal{R}_{A}$, relative to $A$, and let $B$ be a localization satisfying the conclusion of Theorem 5.1.3. Let $C$ be an integral dagger algebra of $M W$-type which is flat over $B$. Then the base change maps $H^{i}\left(M \otimes \mathcal{R}_{B}\right) \otimes_{B} C \cong H^{i}\left(M \otimes \mathcal{R}_{C}\right)$ (induced by the multiplication map $\mathcal{R}_{B} \otimes C \rightarrow \mathcal{R}_{C}$ ) are isomorphisms. Moreover, if we can take $B=A$ and $\mathcal{R}^{\prime \prime}=\mathcal{R}_{A}$ (i.e., $M$ is unipotent), the flatness hypothesis on $C$ can be dropped.

Proof. We first handle the case where $M$ is unipotent, i.e., in case we can take $B=A$ and $\mathcal{R}^{\prime \prime}=\mathcal{R}_{A}$. Let $\mathbf{v}_{1}, \ldots, \mathbf{v}_{n}$ be a unipotent basis, and let $M_{j}$ be the span of $\mathbf{v}_{1}, \ldots, \mathbf{v}_{j}$. Then $\nabla$ carries $M_{j}$ to $M_{j} \otimes \Omega_{\mathcal{R}_{A} / A}^{1}$ and $M_{j} / M_{j+1}$ is isomorphic, as a module with connection, to $\mathcal{R}_{A}$ itself. Thus $H^{i}\left(M_{j} / M_{j+1}\right)$ is a free $A$-module of rank 1 for $i=0,1$, and the map

$$
H^{i}\left(M_{j} / M_{j+1}\right) \otimes_{A} C \rightarrow H^{i}\left(\left(M_{j} / M_{j+1}\right) \otimes \mathcal{R}_{C}\right)
$$

is clearly an isomorphism. We deduce by induction on $j$ and the snake lemma that $H^{i}\left(M_{j}\right) \otimes_{A}$ $C \rightarrow H^{i}\left(M_{j} \otimes \mathcal{R}_{C}\right)$ is an isomorphism, and the case $j=n$ gives what we wanted.

We now treat the general case. Set notation as in Theorem 5.1.3. and put $\mathcal{R}_{C}^{\prime \prime}=\mathcal{R}^{\prime \prime} \otimes_{\mathcal{R}_{B}}$ $\mathcal{R}_{C}$; we then have a commuting diagram

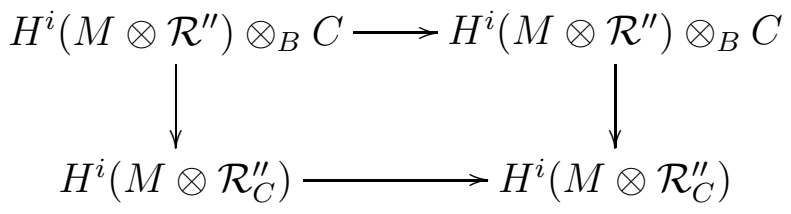

in which the horizontal arrows are projectors induced by the trace map. Since the left vertical arrow is an isomorphism, it induces an isomorphism on the images of the projectors. The image of the lower horizontal arrow is of course $H^{i}\left(M \otimes \mathcal{R}_{C}\right)$. As for the upper horizontal arrow, since $C$ is flat over $B$, tensoring with it commutes with taking images (since the image of $f: X \rightarrow Y$ is the kernel of $Y \rightarrow \operatorname{coker}(f)$, and tensoring with $C$ commutes with taking kernels and cokernels). Since the image of Trace on $H^{i}\left(M \otimes \mathcal{R}^{\prime \prime}\right)$ is $H^{i}\left(M \otimes \mathcal{R}_{B}\right)$, the image of the upper horizontal arrow is thus $H^{i}\left(M \otimes \mathcal{R}_{B}\right) \otimes_{B} C$. We conclude that (17.2.1.1) yields the desired base change isomorphism.

Remark 7.2.2. The same argument works with $C$ replaced by the completion of Frac $B$ for the affinoid topology.

\subsection{Generic pushforwards}

We now exhibit "generic" higher direct images in a simple geometric setting, using the formalism of [Cr, 8.1]. 
Hypothesis 7.3.1. Let $A$ be a dagger algebra of MW-type with special fibre $X$. Let $f: A \rightarrow A\langle x\rangle^{\dagger}$ be the canonical inclusion, and embed $A\langle x\rangle^{\dagger}$ into $\mathcal{R}_{A}$ by mapping $\sum c_{i} x^{i}$ to $\sum c_{i} t^{-i}$.

Definition 7.3.2. Let $M$ be a $(\sigma, \nabla)$-module over $A\langle x\rangle^{\dagger}$, and let $\nabla_{v}$ denote the composition

$$
M \stackrel{\nabla}{\rightarrow} M \otimes \Omega_{A\langle x\rangle^{\dagger} / K}^{1} \rightarrow M \otimes \Omega_{A\langle x\rangle^{\dagger} / A}^{1}=M \otimes d x .
$$

Let

$$
\begin{aligned}
& \nabla_{v}^{\text {loc }}: M \otimes \mathcal{R}_{A} \rightarrow M \otimes \Omega_{\mathcal{R}_{A} / A}^{1} \\
& \nabla_{v}^{\text {qu }}:\left(M \otimes \mathcal{R}_{A}\right) / M \rightarrow\left(M \otimes \Omega_{\mathcal{R}_{A} / A}^{1}\right) /\left(M \otimes \Omega_{A\langle x\rangle^{\dagger} / A}^{1}\right)
\end{aligned}
$$

be the maps induced by $\nabla_{v}$. We then define the following $A$-modules:

$$
\begin{array}{cl}
R^{0} f_{*} M=\operatorname{ker}\left(\nabla_{v}\right), & R^{1} f_{*} M=\operatorname{coker}\left(\nabla_{v}\right) \\
R_{\mathrm{loc}}^{0} f_{*} M=\operatorname{ker}\left(\nabla_{v}^{\mathrm{loc}}\right), & R_{\mathrm{loc}}^{1} f_{*} M=\operatorname{coker}\left(\nabla_{v}^{\mathrm{loc}}\right) \\
R^{1} f_{!} M=\operatorname{ker}\left(\nabla_{v}^{\text {qu }}\right), & R^{2} f_{!} M=\operatorname{coker}\left(\nabla_{v}^{\text {qu }}\right) .
\end{array}
$$

The snake lemma applied to the diagram with exact rows

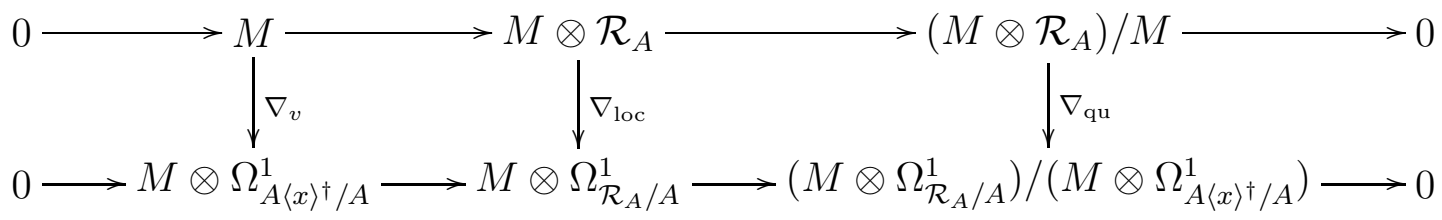

gives an exact sequence

$$
0 \rightarrow R^{0} f_{*} M \rightarrow R_{\mathrm{loc}}^{0} f_{*} M \rightarrow R^{1} f_{!} M \rightarrow R^{1} f_{*} M \rightarrow R_{\mathrm{loc}}^{1} f_{*} M \rightarrow R^{2} f_{!} M \rightarrow 0
$$

using which we can define the "primitive middle cohomology":

$$
R_{p}^{1} f_{*} M=\operatorname{im}\left(R^{1} f_{!} M \rightarrow R^{1} f_{*} M\right) .
$$

The Frobenius on $M$ acts on all of these modules. Moreover, if we write $\nabla_{h}$ for the composition

$$
M \stackrel{\nabla}{\rightarrow} M \otimes \Omega_{A\langle x\rangle^{\dagger} / K}^{1} \rightarrow M \otimes\left(A\langle x\rangle^{\dagger} \otimes_{A} \Omega_{A / K}^{1}\right)=M \otimes_{A} \Omega_{A / K}^{1},
$$

then each module $N$ listed above admits a $K$-linear connection $\nabla_{h}: N \rightarrow N \otimes_{A} \Omega_{A / K}^{1}$. Note that this is true even if $N$ is not finitely generated; that is because $\Omega_{A / K}^{1}$ is projective over $A$, so computing the kernel/cokernel of any of $\nabla_{v}, \nabla_{v}^{\text {loc }}, \nabla_{v}^{\text {qu }}$ commutes with tensoring with $\Omega_{A / K}^{1}$.

We now prove our main theorem on higher direct images. Note the essential use of the nonrelative case (Theorem 6.4.2). 
Theorem 7.3.3. Let $A$ be an integral dagger algebra of $M W$-type, and let $f: A \rightarrow A\langle x\rangle^{\dagger}$ denote the canonical inclusion. Let $M$ be a $(\sigma, \nabla)$-module over $A\langle x\rangle^{\dagger}$. Then after replacing $A$ by a suitable localization, the modules $R^{i} f_{*} M, R_{\mathrm{loc}}^{i} f_{*} M, R^{i} f_{!} M, R_{p}^{1} f_{*} M$ become $(\sigma, \nabla)$-modules over A.

Proof. Choose a nonzero $g \in A\langle x\rangle^{\dagger}$ such that $M$ becomes free over $A\left[g^{-1}\right]$. Write $g=$ $\sum_{i=0}^{\infty} g_{i} x^{i}$ and choose the largest $i$ for which $\left|g_{i}\right|$ is maximized. By Lemma 2.5.13, the series expansion of $\left(1-\left(1-g /\left(g_{i} x^{i}\right)\right)\right)^{-1}$ converges in $\mathcal{R}_{A\left\langle g_{i}^{-1}\right\rangle^{+}}$; hence for any localization $B$ of $A\left\langle g_{i}^{-1}\right\rangle^{\dagger}, g$ is invertible in $\mathcal{R}_{B}^{\text {int }}\left[\frac{1}{p}\right]$. In particular, $M \otimes \mathcal{R}_{B}$ is free for such $B$, and so we can choose $B$ satisfying the conclusion of Theorem 5.1.3.

We first verify that over $B, R^{i} f_{*} M, R_{\mathrm{loc}}^{i} f_{*} M, R^{i} f_{!} M, R_{p}^{1} f_{*} M$ become finitely generated $B$-modules. We have that $R_{\mathrm{loc}}^{0} f_{*} M$ and $R_{\mathrm{loc}}^{1} f_{*} M$ are finitely generated by Proposition 7.1.4 by the exactness of (7.3.2.2), it suffices to check that $R_{p}^{1} f_{*} M$ is finitely generated.

Let $L$ be the completion of Frac $A$ for the affinoid topology and put $M_{L}=M \otimes L\langle x\rangle^{\dagger}$. By Theorem 6.4.2 and Proposition 7.1.4 respectively, $R^{1} f_{*} M_{L}$ and $R_{\mathrm{loc}}^{0} f_{*} M_{L}$ are finite dimensional over $L$; by the snake lemma, so then is $R^{1} f_{!} M_{L}$. Now view $\left(M \otimes \mathcal{R}_{A}\right) / M$ and $\left(M_{L} \otimes \mathcal{R}_{L}\right) / M_{L}$ as $A$-submodules of direct products of copies of $A$ and $L$, respectively, indexed by pairs consisting of a positive power of $t$ and a basis element of $M$. In particular, we may view $\left(M \otimes \mathcal{R}_{A}\right) / M$ as an $A$-submodule of $\left(M_{L} \otimes \mathcal{R}_{L}\right) / M_{L}$; in this point of view, $R^{1} f_{!} M$ is none other than the intersection of $R^{1} f_{!} M_{L}$ with $\left(M \otimes \mathcal{R}_{A}\right) / M$ within $\left(M_{L} \otimes \mathcal{R}_{L}\right) / M_{L}$. Since $A$ is noetherian, we may apply Lemma 7.3 .4 below to deduce that $R^{1} f_{!} M$ is finitely generated, as then is $R_{p}^{1} f_{*} M$.

For $N$ any one of the modules under consideration, we now know that $N$ is finitely generated. Since $N$ is equipped with a connection given by $\nabla_{h}, N$ is also locally free over $B$ by Lemma 3.3.4. We also have an action of $F$ on $N$, which is injective by Remark 3.4.4. The quotient of $N$ by the submodule generated by the image of $F$ also comes equipped with a connection, so it is locally free. However, the rank of this quotient must be zero, so the quotient itself must be zero. Hence the image of $F$ generates $N$; that is, the induced $B$-linear map $\sigma_{A}^{*} N \rightarrow N$ is surjective. Now the kernel of this map is equipped with a connection, so is locally free by Lemma 3.3 .4 and necessarily of rank zero, hence trivial. Thus $\sigma_{A}^{*} N \rightarrow N$ is an isomorphism.

We conclude that all of the modules in question become $(\sigma, \nabla)$-modules over $B$, as desired.

Lemma 7.3.4. Let $R$ be a noetherian domain, let $L$ be a field containing $R$, and let $S$ be an arbitrary set. Let $N$ be a finite dimensional L-subspace of the direct product $L^{S}$. Then $N \cap R^{S}$ is a finitely generated $R$-module.

Proof. We induct on the dimension of the $L$-span of $N \cap R^{S}$. If this dimension is zero, then $N \cap R^{S}=0$ and there is nothing to check. Otherwise, we may pick $s \in S$ such that, writing $\pi_{s}$ for the projection of $R^{S}$ onto the $s$-component, we have $\pi_{s}\left(N \cap R^{S}\right) \neq 0$. Now $\pi_{s}\left(N \cap R^{S}\right)$ is an ideal of $R$, which by the noetherian hypothesis must be finitely generated. Pick elements $\mathbf{v}_{1}, \ldots, \mathbf{v}_{n} \in N \cap R^{S}$ whose images under $\pi_{s}$ generate $\pi_{s}\left(N \cap R^{S}\right)$. Then each 
element of $N \cap R^{S}$ can be written as an $R$-linear combination of $\mathbf{v}_{1}, \ldots, \mathbf{v}_{n}$ plus an element of $\left(N \cap \pi_{s}^{-1}(0)\right) \cap R^{S}$, so it suffices to check that the latter is finitely generated. But the latter has the same form as the original intersection except with $N$ replaced by $N \cap \pi_{s}^{-1}(0)$, and the dimension of the $L$-span of $\left(N \cap \pi_{s}^{-1}(0)\right) \cap R^{S}$ has gone down by one since $\pi_{s}\left(N \cap R^{S}\right) \neq 0$. Thus the induction hypothesis yields the desired result.

Remark 7.3.5. By passing down an étale map to this geometric situation (using Proposition 9.1.2), one can use these methods to construct "generic" higher direct images for a family of affine curves. (By contrast, Tsuzuki [T3] constructs generic higher direct images for families of projective curves, using more global techniques.) On the other hand, pushforwards with compact supports are more difficult to construct, and we do not believe our technique is sophisticated enough to handle them. In particular, in the "non-generic" setting, the objects we call $R^{i} f_{!} M$ may not agree with the direct image with compact supports in the category of arithmetic $\mathcal{D}$-modules.

Remark 7.3.6. Although our argument essentially uses the presence of the Frobenius structure, in order to invoke the $p$-adic local monodromy theorem, it is conceivable that this dependence could be lifted. To do so, it may be necessary to extend the $p$-adic index theory of Christol and Mebkhout CM2 to cover modules with connection over the Robba ring over a dagger algebra.

\subsection{A Leray spectral sequence}

The value of the pushforward construction is that the cohomology of a $(\sigma, \nabla)$-module can be controlled using the cohomology of its pushforwards, as follows.

Proposition 7.4.1. Let $A$ be a dagger algebra, and let $M$ be a $(\sigma, \nabla)$-module over $A\langle x\rangle^{\dagger}$. Let $P$ and $Q$ be the kernel and cokernel, respectively, of

$$
\nabla_{v}: M \stackrel{\nabla}{\rightarrow} M \otimes_{A\langle x\rangle^{\dagger}} \Omega_{A\langle x\rangle^{\dagger} / K}^{1} \rightarrow M \otimes_{A\langle x\rangle^{\dagger}} \Omega_{A\langle x\rangle^{\dagger} / A}^{1}
$$

Then there is a long exact sequence

$$
\cdots \rightarrow H^{i}(P) \rightarrow H^{i}(M) \rightarrow H^{i-1}(Q) \rightarrow H^{i+1}(P) \rightarrow \cdots .
$$

Proof. The claim is a formal consequence of the Leray spectral sequence associated to the double complex

$$
E_{0}^{p q}=M \otimes_{A} \Omega_{A / K}^{p} \otimes_{K\langle x\rangle^{\dagger}} \Omega_{K\langle x\rangle^{\dagger} / K}^{q}
$$

whose total cohomology computes $H^{i}(M)$. (We follow [BT, Section 14] in our terminology concerning spectral sequences.) Using the flatness of $\Omega_{A / K}^{i}$ over $A$, we have

$$
\begin{gathered}
E_{1}^{p 0}=P \otimes \Omega_{A / K}^{p}, E_{1}^{p 1}=Q \otimes \Omega_{A / K}^{p} \\
E_{2}^{p 0}=H^{p}(P), E_{2}^{p 1}=H^{p}(Q),
\end{gathered}
$$

the differential $d_{2}$ maps $E_{2}^{p q}$ to $E_{2}^{(p+2)(q-1)}$, and the sequence degenerates at $E_{3}$ (that is, $\left.d_{3}=d_{4}=\cdots=0\right)$. Thus in the desired long exact sequence, the maps $H^{i-1}(Q) \rightarrow H^{i+1}(P)$ are given by $d_{2}$; the remaining maps come from the fact that $E_{3}$ abuts to $H^{i}(M)$. 


\subsection{Pushforwards and base change}

To establish the Künneth formula, we need to prove that our relative pushforwards commute with flat base change. The argument requires a bit of $p$-adic functional analysis; since this will be introduced in Chapter 8 , the proof will contain a forward reference, but it can be verified that no vicious circle has been created.

Lemma 7.5.1. Let $A$ be an integral dagger algebra of $M W$-type, and let $M$ be a $(\sigma, \nabla)$ module over $A\langle x\rangle^{\dagger}$, relative to $A$. Then there exists a localization $A^{\prime}$ of $A$ for which the image of $\nabla$ on $M \otimes_{A\langle x\rangle^{\dagger}} A^{\prime}\langle x\rangle^{\dagger}$ is closed for the fringe topology.

Proof. This is a corollary of Proposition 8.4.4. Note that the proposition includes the conclusion that $\nabla$ is strict; although we do not need that conclusion here, its presence is a key aspect of the proof.

We can now establish a flat base change theorem.

Proposition 7.5.2. Let $A$ and $B$ be integral dagger algebras of $M W$-type, with $B$ flat over $A$. Let $f: A \rightarrow A\langle x\rangle^{\dagger}$ and $g: B \rightarrow B\langle x\rangle^{\dagger}$ be the canonical inclusions. Let $M$ be a $(\sigma, \nabla)$-module over $A\langle x\rangle^{\dagger}$, and put $M_{B}=M \otimes_{A\langle x\rangle^{\dagger}} B\langle x\rangle^{\dagger}$. Suppose that the conclusion of Theorem 7.3 .3 holds for $M$ without further localization. Then the canonical base change morphisms

$$
\begin{aligned}
\left(R^{i} f_{*} M\right) \otimes_{A} B & \rightarrow R^{i} g_{*} M_{B} \\
\left(R_{\mathrm{loc}}^{i} f_{*} M\right) \otimes_{A} B & \rightarrow R_{\mathrm{loc}}^{i} g_{*} M_{B} \\
\left(R^{i} f_{!} M\right) \otimes_{A} B & \rightarrow R^{i} g_{!} M_{B} \\
\left(R_{p}^{1} f_{*} M\right) \otimes_{A} B & \rightarrow R_{p}^{1} g_{*} M_{B}
\end{aligned}
$$

are isomorphisms. In particular, the conclusion of Theorem 7.3 .3 holds for $M_{B}$ without further localization.

Proof. For $R_{\text {loc }}^{i}$, this follows from Proposition [7.2.1. As in Theorem [7.3.3, we have that $R_{p}^{1} g_{*} M_{B}=\operatorname{im}\left(R^{1} g_{!} M_{B} \rightarrow R^{1} g_{*} M_{B}\right)$ is finitely generated, which shows (by the exactness of (7.3.2.2) ) that all of the pushforwards are finitely generated without further localization.

It is clear that $\left(R^{0} f_{*} M\right) \otimes_{A} B \rightarrow R^{0} g_{*} M_{B}$ and $\left(R^{1} f_{!} M\right) \otimes_{A} B \rightarrow R^{1} g_{!} M_{B}$ are injective, since the map of complexes $M \otimes \Omega_{A\langle x\rangle^{\dagger} / A} \otimes_{A} B \rightarrow M_{B} \otimes \Omega_{B\langle x\rangle^{\dagger} / B}$ is injective and $R^{0} f_{*} M$ and $R^{1} f_{!} M$ are defined by taking certain kernels of $\nabla$.

We next show that $\left(R^{1} f_{*} M\right) \otimes_{A} B \rightarrow R^{1} g_{*} M_{B}$ is surjective; since source and target are $\nabla$-modules, surjectivity may be checked after localizing $B$ (and this localization preserves flatness over $A$ ). In particular, we may reduce to the case where the conclusion of Lemma 7.5 .1 holds for $N=M_{B} \otimes \Omega_{B\langle x\rangle^{\dagger} / B}^{1}$ without further localization. For brevity, put $N_{1}=M \otimes \Omega_{A\langle x\rangle^{\dagger} / A}^{1} \otimes_{A} B$, let $N_{2}$ be the image of $\nabla$ on $M_{B}$. Then $N_{1}$ is dense in $N$, and $N_{2}$ is closed in $N$ by Lemma 7.5.1. Since $B$ is noetherian and $R^{1} g_{*} M_{B}=N / N_{2}$ is finitely generated over $B$, the image of $N_{1}$ in $N / N_{2}$ is also finitely generated. Thus $N_{1}+N_{2}$ is closed 
in $N$; however, it is also dense because $N_{1}$ already is. Consequently $N_{1}+N_{2}=N$, and we deduce that $\left(R^{1} f_{*} M\right) \otimes_{A} B \rightarrow R^{1} g_{*} M_{B}$ is surjective.

To conclude, write down the snake lemma exact sequences (7.3.2.2) corresponding to $M$ and $M_{B}$. Then the base change maps give a map between the former, tensored with $B$, and the latter, and we are in the situation of the following Lemma 7.5.3. It follows that all of the base change maps are isomorphisms.

Lemma 7.5.3. Suppose in the category of abelian groups, the diagram

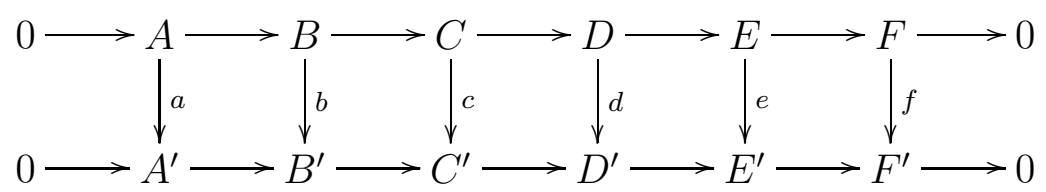

commutes and has exact rows. Also suppose that $c$ is injective, $b$ and $e$ are isomorphisms, and $d$ is surjective. Then all six vertical arrows are isomorphisms.

Proof. Apply the five lemma first to $c$ and $d$, then to $a$ and $f$.

Remark 7.5.4. As in Remark 7.2.2, we can also take $B$ to be the completion of Frac $A$ under the affinoid topology. This implies that the pushforwards all have the "correct" ranks, namely the ranks over the generic fibre.

\subsection{Pushforwards and external products}

Using flat base change, we can now verify that our pushforward construction commutes with external products. This will be crucial for verifying the Künneth decomposition.

Lemma 7.6.1. Let $A$ be an integral dagger algebra of $M W$-type, and let $f: A \rightarrow A\langle x\rangle^{\dagger}$ be the natural inclusion. Let $M$ be a $(\sigma, \nabla)$-module over $A\langle x\rangle^{\dagger}$, and let $N$ be a $(\sigma, \nabla)$-module over $A$. Then the natural maps

$$
\begin{aligned}
\left(R^{i} f_{*} M\right) \otimes_{A} N & \rightarrow R^{i} f_{*}\left(M \otimes_{A} N\right) \\
\left(R_{\mathrm{loc}}^{i} f_{*} M\right) \otimes_{A} N & \rightarrow R_{\mathrm{loc}}^{i} f_{*}\left(M \otimes_{A} N\right) \\
\left(R^{i} f_{!} M\right) \otimes_{A} N & \rightarrow R^{i} f_{!}\left(M \otimes_{A} N\right) \\
\left(R_{p}^{1} f_{*} M\right) \otimes_{A} N & \rightarrow R_{p}^{1} f_{*}\left(M \otimes_{A} N\right)
\end{aligned}
$$

are isomorphisms.

Proof. All of the pushforwards are computed as kernels and cokernels of various $A$-module homomorphisms. Since $N$ is flat over $A$, these computations commute with tensoring with $N$.

Given modules $M$ and $N$ over dagger algebras $A$ and $B$, respectively, define the external product of $M$ and $N$ as the $(A \widehat{\otimes} B)$-module

$$
M \otimes_{A, B} N=M \otimes_{A}(A \widehat{\otimes} B) \otimes_{B} N .
$$


Proposition 7.6.2. Let $A$ and $B$ be integral dagger algebras of $M W$-type. Let $M$ be a $(\sigma, \nabla)$-module over $A\langle x\rangle^{\dagger}$ and let $N$ be $a(\sigma, \nabla)$-module over $B$. Let $f: A \rightarrow A\langle x\rangle^{\dagger}$ and $g$ : $A \widehat{\otimes} B \rightarrow(A \widehat{\otimes} B)\langle x\rangle^{\dagger}$ be the natural inclusions. Suppose that the conclusion of Theorem 7.3.3 holds for $M$ without further localization. Then the natural maps

$$
\left(R^{i} f_{*} M\right) \bigotimes_{A, B} N \rightarrow R^{i} g_{*}\left(M \bigotimes_{A\langle x\rangle^{\dagger}, B} N\right)
$$

are isomorphisms.

Again, the truth of Theorem 7.3 .3 for $M \otimes_{A\langle x\rangle^{\dagger}, B} N$ without further localization is part of the conclusion.

Proof. Note that $A \widehat{\otimes} B$ is flat over $A$ by Lemma 2.2 .3 (see Remark 2.3.9). Hence we can apply Proposition 7.5 .2 to deduce that the natural maps

$$
\left(R^{i} f_{*} M\right) \bigotimes_{A, B} N \rightarrow\left(R^{i} g_{*}\left(M \otimes_{A\langle x\rangle^{\dagger}}(A \widehat{\otimes} B)\langle x\rangle^{\dagger}\right)\right) \otimes_{B} N
$$

are isomorphisms. By Lemma 7.6.1, the natural maps

$$
\left(R^{i} g_{*}\left(M \otimes_{A\langle x\rangle^{\dagger}}(A \widehat{\otimes} B)\langle x\rangle^{\dagger}\right)\right) \otimes_{B} N \rightarrow R^{i} g_{*}\left(M \otimes_{A\langle x\rangle^{\dagger}, B} N\right)
$$

are also isomorphisms. Putting these isomorphisms together yields the desired result.

\section{Cohomology with compact supports}

In this chapter, we review Berthelot's construction of cohomology with compact supports, then make this construction explicit in the case of affine space. The resulting computation has a flavor similar to that of the Monsky-Washnitzer cohomology we have been using so far; it also bears a passing resemblance to Dwork's "dual theory". We then verify Poincaré duality on affine space; this requires some of the machinery of $p$-adic functional analysis, for which we draw from Crew $\mathrm{Cr}$.

\subsection{The cohomology of affine space}

We now restrict consideration to a simple geometric situation.

Hypothesis 8.1.1. Put $X=\mathbb{A}_{k}^{n}$ and $\bar{X}=\mathbb{P}_{k}^{n}$, and let $P$ be the formal completion of $\mathbb{P}_{\mathcal{O}}^{n}$ along $\mathbb{P}_{k}^{n}$. We can identify $\tilde{P}$ with the set of tuples $\left(z_{0}, \ldots, z_{n}\right)$ over $\mathcal{O}^{\text {alg }}$ (the integral closure of $\mathcal{O}$ in the algebraic closure $K^{\text {alg }}$ of $K$ ) with $\max \left\{\left|z_{i}\right|\right\}=1$ modulo the action of $\left(\mathcal{O}^{\text {alg }}\right)^{*}$ and of $\operatorname{Gal}\left(K^{\text {alg }} / K\right)$. We use the Frobenius lift $\sigma$ sending $\left(z_{0}, \ldots, z_{n}\right)$ to $\left(z_{0}^{q}, \ldots, z_{n}^{q}\right)$.

Definition 8.1.2. For $i=0, \ldots, n$, let $U_{i}$ be the subspace of $\tilde{P}$ on which $\left|z_{i}\right|=1$. Then each $U_{i}$ is an affinoid subdomain of $\tilde{P}$, and

$$
\Gamma\left(U_{i}\right)=K\left\langle\frac{z_{0}}{z_{i}}, \ldots, \frac{z_{i-1}}{z_{i}}, \frac{z_{i+1}}{z_{i}}, \ldots, \frac{z_{n}}{z_{i}}\right\rangle .
$$

For $S \subseteq\{0, \ldots, n\}$, put $U_{S}=\cap_{i \in S} U_{i}$. 
Definition 8.1.3. For $\rho>1$ in the norm group of $K^{\text {alg }}$, let $V_{\rho}$ be the subspace of $\tilde{P}$ on which $\left|z_{0}\right| \geq \rho^{-1}$. Then $V_{\rho}$ is a strict neighborhood of $] X$, and is an affinoid: namely, if we put $x_{i}=z_{i} / z_{0}$, then $V_{\rho}=\operatorname{Maxspec} T_{n, \rho}$.

We now want to compute $\mathbb{R} \underline{\Gamma}(\mathcal{F})$ (as defined in Definition 4.3.4) for $\mathcal{F}$ the coherent sheaf associated to a finite locally free $T_{n, \rho}$-module $E$, viewed as a one-term complex in degree 0. Our model for the computation is Be2, Exemple 3.4], but our situation is complicated by the fact that $V_{\rho} \backslash U_{0}$ is not quasi-Stein if $n>1$. However, it admits a nice cover by quasi-Stein varieties. (This fact is alluded to in the proof of [Be6, Proposition 1.1].)

Definition 8.1.4. For $T \subseteq\{1, \ldots, n\}$, let $W_{T}$ be the subspace of $V_{\rho}$ on which $\left|x_{i}\right|>1$ for $i \in T$.

Lemma 8.1.5. The rigid analytic space $W_{T}$ is quasi-Stein for each $T$.

Proof. For $\eta \in(1, \rho) \cap \Gamma^{*}$, let $X_{\eta}$ be the subset of $W_{T}$ on which $\eta \leq\left|x_{i}\right| \leq \rho$ for $i \in T$. Then $X_{\eta}$ is an affinoid, $X_{\eta} \subseteq X_{\tau}$ if $\eta>\tau$, and $W_{T}$ is the union of the $X_{\eta}$. The density of $\Gamma\left(X_{\tau}\right)$ in $\Gamma\left(X_{\eta}\right)$ is straightforward, as in fact the Laurent polynomial ring $K\left[x_{1}, \ldots, x_{n}\right]\left[x_{i}^{-1}: i \in T\right]$ is dense in $\Gamma\left(X_{\eta}\right)$. Thus $W_{T}$ is quasi-Stein, as claimed.

We do not have names for the coordinate rings of the $W_{T}$, so we introduce them now.

Definition 8.1.6. Put $\mathcal{S}_{r}^{(n)}=\mathcal{R}_{K, r}^{\left\{t_{1}, \ldots, t_{n}\right\}}$. For $r=\log _{p}(\rho)$, we may identify $T_{n, \rho}$ as a subring of $\mathcal{S}_{r}^{(n)}$ by equating $\sum_{I} c_{I} x^{I}$ with $\sum_{I} c_{I} t^{-I}$. For $T \subset\{1, \ldots, n\}$, let $\mathcal{S}_{r}^{(n), T}$ denote the subring of the ring $\mathcal{S}_{r}^{(n)}$ of series in which the variable $t_{i}$ may occur with a positive power if and only if $i \in T$. For short, write $i^{\prime}$ for the set $\{1, \ldots, n\} \backslash\{i\}$. Observe that $\Gamma\left(W_{T}\right)=\mathcal{S}_{r}^{(n), T}$ and that $\mathcal{S}_{r}^{(n), \emptyset}=T_{n, \rho}$.

Recall that the complex $\mathbb{R} \underline{\Gamma}(\mathcal{F})$ is isomorphic, in the derived category, to the two-term complex

$$
0 \rightarrow \mathcal{F} \rightarrow \iota_{*} \iota^{*} \mathcal{F} \rightarrow 0
$$

We now compute the (hyper)cohomology of this complex.

Proposition 8.1.7. Let $\mathcal{F}$ be the coherent sheaf associated to the finite locally free $T_{n, \rho^{-}}$ module E. Then the cohomology of (8.1.6.1) is equal to

$$
\frac{E \otimes \mathcal{S}_{r}^{(n)}}{\sum_{i} E \otimes \mathcal{S}_{r}^{(n), i^{\prime}}}
$$

in degree $n$ and zero elsewhere.

Note in particular that this space does not depend on $r$. 
Proof. We use the admissible covering $U_{0}, U_{1} \cap V_{\rho}, \ldots, U_{n} \cap V_{\rho}$ of $V_{\rho}$ to resolve the two terms of (8.1.6.1) with Cech complexes. For each nonempty subset $T$ of $\{1, \ldots, n\}$, we have

$$
\left(V_{\rho} \backslash U_{0}\right) \cap \bigcap_{j \in T} U_{j}=W_{T}
$$

which by Lemma 8.1.5 is quasi-Stein; also, each $U_{S}$ is affinoid and hence quasi-Stein. Hence (by Theorem B again) we may replace the Cech complexes with their global sections. In other words, the complex (8.1.6.1) is isomorphic in the derived category to the simple complex associated to the double complex with rows

$$
\bigoplus_{i}\left(\oplus_{S} \Gamma\left(\mathcal{F}, U_{S}\right)\right) \rightarrow \bigoplus_{i}\left(\oplus_{T} \Gamma\left(\mathcal{F}, W_{T}\right)\right)
$$

where $S$ runs over all subsets of $\{0,1, \ldots, n\}$ of size $i$ and $T$ runs over all subsets of $\{1, \ldots, n\}$ of size $i$.

We now compute the cohomology of (8.1.7.1) using the Leray spectral sequence (which already arose in Section (7.4). Half of the computation of the $E_{1}$ term is easy: we have $E_{1}^{00}=E$ and $E_{1}^{0 q}=0$ for $q>0$. To compute the rest, we use the fact that the $j$-th cohomology of the Čech complex with $i$-th term

$$
\bigoplus_{i}\left(\oplus_{T} \mathcal{S}_{r}^{(n), T}\right)
$$

is given by

$$
H^{j}= \begin{cases}T_{n, \rho} & j=0 \\ \mathcal{S}_{r}^{(n)} / \sum_{i} \mathcal{S}_{r}^{(n), i^{\prime}} & j=n-1 \\ 0 & \text { otherwise. }\end{cases}
$$

This is straightforward to prove by induction on $n$, if notationally cumbersome; we leave details to the reader. The upshot is that $E_{1}^{10}=E, E_{1}^{1(n-1)}=\left(E \otimes \mathcal{S}_{r}^{(n)}\right) / \sum_{i}\left(E \otimes \mathcal{S}_{r}^{n, i^{\prime}}\right)$ and $E_{1}^{1 q}=0$ for $q \neq 0, n-1$.

Recall that $E_{2}^{p q}$ is the $p$-th cohomology of the complex $E_{1}^{q}$. For $q=0$, the complex is just $E \rightarrow E$ via the identity map, so $E_{2}^{p 0}=0$ for all $p$. The only other term left is $E_{2}^{1(n-1)}=E_{1}^{1(n-1)}$. Thus the spectral sequence degenerates at $E_{2}$, yielding the desired result.

We can now compute the cohomology with compact supports of an overconvergent $F$ isocrystal on $\mathbb{A}^{n}$.

Proposition 8.1.8. Let $\mathcal{E}$ be an overconvergent $F$-isocrystal on $\mathbb{A}^{n}$ which corresponds to a $(\sigma, \nabla)$-module $M$ over $T_{n, \rho}$. Then

$$
H_{c, \text { rig }}^{n+j}\left(\mathbb{A}^{n} / K, \mathcal{E}\right)=H^{j}\left(\left(M \otimes \Omega \otimes \mathcal{S}_{r}^{(n)}\right) / \oplus_{i}\left(M \otimes \Omega \otimes \mathcal{S}_{r}^{(n), i^{\prime}}\right)\right)
$$


Proof. The cohomology is again computed by a Leray spectral sequence. This time, by Proposition 8.1.7, we have

$$
E_{1}^{p n}=\left(M \otimes \Omega^{p} \otimes \mathcal{S}_{r}^{(n)}\right) / \oplus_{i}\left(M \otimes \Omega^{p} \otimes \mathcal{S}_{r}^{(n), i^{\prime}}\right)
$$

and $E_{1}^{p q}=0$ for $q \neq n$. Thus the spectral sequence degenerates at $E_{2}$ and yields the desired result.

Corollary 8.1.9. The space $H_{c, \text { rig }}^{2 n}\left(\mathbb{A}^{n} / K\right)$ is one-dimensional over $K$.

Proof. It is clear from the description in Proposition 8.1.8 that $H_{c, \text { rig }}^{2 n}\left(\mathbb{A}^{n} / K\right)$ is generated by the class of $d t_{1} / t_{1} \wedge \cdots \wedge d t_{n} / t_{n}$.

\subsection{The Poincaré pairing}

We next recall the formalism of the Poincaré pairing, and explicate this formalism over affine space. Our discussion is drawn from [Be6].

Retain Hypothesis 4.2.1, and assume now that $X$ has dimension $n$. Let $j$ denote the inclusion of $] X$ [ into a strict neighborhood $V$. As in [Be6, Lemme 2.1], for any sheaves $\mathcal{F}, \mathcal{G}$ of $K$-vector spaces on $V$, one has an isomorphism

$$
\operatorname{Hom}\left(j^{\dagger} \mathcal{F}, \mathcal{G}\right) \cong \underline{\Gamma}\left(\operatorname{Hom}_{K}(\mathcal{F}, \mathcal{G})\right) .
$$

If $\mathcal{F}, \mathcal{G}$ are overconvergent $F$-isocrystals, we thus obtain a pairing in cohomology

$$
H_{\text {rig }}^{j}(X / K, \mathcal{F}) \otimes_{K} H_{c, \text { rig }}^{l}(X / K, \mathcal{G}) \rightarrow H_{c, \text { rig }}^{j+l}(X / K, \mathcal{F} \otimes \mathcal{G}) .
$$

In particular, if $\mathcal{G}=\mathcal{F}^{\vee}$ is the dual of $\mathcal{F}$, we have a natural morphism from $\mathcal{F} \otimes \mathcal{F}^{\vee}$ to the constant $F$-isocrystal. This yields a map

$$
H_{c, \text { rig }}^{2 n}\left(X / K, \mathcal{F} \otimes \mathcal{F}^{\vee}\right) \rightarrow H_{c, \text { rig }}^{2 n}(X / K) .
$$

One can now construct a trace morphism from $H_{c, \text { rig }}^{2 n}(X / K)$ to $K$. In [Be6, 1.2], this is done by a reduction to the corresponding construction in coherent cohomology; one then relates the construction to crystalline cohomology [Be6, 1.5] to check that the trace is an isomorphism when $X$ is geometrically irreducible. We will check the latter instead by reducing to the case of affine space (Lemma 9.3.2); in any case, we already have this for $X=\mathbb{A}^{n}$ by Corollary 8.1.9, which will suffice for the moment.

\subsection{Review of $p$-adic functional analysis}

To make more progress, we need some basic facts from $p$-adic functional analysis; we state the relevant results here and refer to $[\mathrm{Cr}]$ (or $[\mathrm{Sc}$ ) for further discussion. For brevity, in this section we use the word "space" to mean "topological vector space over $K$ ".

Definition 8.3.1. Define a subset $S$ of a space $V$ to be 
- convex if for any $\mathbf{v}, \mathbf{w}, \mathbf{x} \in S$ and any $a, b, c \in \mathcal{O}$ with $a+b+c=1, a \mathbf{v}+b \mathbf{w}+c \mathbf{x} \in S$;

- balanced if $0 \in S$ (normally only said of convex sets);

- absorbing if for any $\mathbf{v} \in V$, we have $\mathbf{v} \in \lambda S$ for some $\lambda \in K$;

- bounded if for any open neighborhood $U$ of zero, we have $S \subseteq \lambda U$ for some $\lambda \in K$;

- linearly compact if $S$ is convex, and every convex filter on $S$ (i.e., every filter admitting a base of convex sets) has an accumulation point.

Definition 8.3.2. Define a space $V$ to be

- locally convex if its zero element admits a neighborhood basis consisting of convex subsets;

- Fréchet if it is Hausdorff and complete, and its topology is induced by a countable collection of seminorms (and hence is locally convex);

- $L F$ (for "limit-of-Fréchet") if it is separated and locally convex, and is a direct limit of Fréchet spaces;

- barreled if any closed, convex, balanced, absorbing subset is a neighborhood of zero;

- Montel if it is barreled, and every closed convex bounded subset is linearly compact.

Definition 8.3.3. The strong dual of a space $V$ is the space $V^{\vee}$ of continuous linear maps $f: V \rightarrow K$, topologized by decreeing a basis of open sets of the form $\{f: V \rightarrow K \mid f(B) \subseteq U\}$ for $B \subseteq V$ bounded and $U \subseteq K$ open.

Definition 8.3.4. A continuous linear map $f: V \rightarrow W$ of spaces is strict if the induced continuous bijection $V / \operatorname{ker}(f) \rightarrow \operatorname{im}(f)$ is a homeomorphism. As noted in [Cr, 3.7], given a finite collection of continuous linear maps $f_{i}: V_{i} \rightarrow W_{i}$ between locally convex spaces, the map $\oplus f_{i}: \oplus V_{i} \rightarrow \oplus W_{i}$ is strict (resp. strict with closed image) if and only if each $f_{i}$ is strict (resp. strict with closed image).

The notion of strictness is somewhat delicate: for example, a composition $g \circ f$ of strict maps with closed image is only guaranteed to be strict with closed image if $f$ is surjective or $g$ is injective. Otherwise it may not even be strict, as in the example (suggested by the referee)

$$
K\langle x\rangle \rightarrow K\langle x, y\rangle \rightarrow K\langle x, y\rangle /(x-p y) \cong K\langle y\rangle .
$$

A number of useful criteria for strictness appear in [Cr, $\S 3]$; here is one of them [Cr, Corollary 3.12].

Proposition 8.3.5. Let $f: V \rightarrow W$ be a continuous linear map between Fréchet-Montel spaces. Then $f$ is strict if and only if $f^{\vee}: W^{\vee} \rightarrow V^{\vee}$ is strict. 
We also need the following form of the Hahn-Banach theorem; see for example Sc, Corollary 9.4] (or [Mn, Théorème V.2]). Note that we need here the fact that the valuation on $K$ is discrete, so that $K$ is spherically complete. Note also that we will only use this result in case $W$ is closed.

Proposition 8.3.6. Let $V$ be a locally convex space, let $W$ be a subspace of $V$, and let $f: W \rightarrow K$ a continuous linear map. Then there exists a continuous linear map $g: V \rightarrow K$ extending $f$.

\subsection{Topology of $(\sigma, \nabla)$-modules}

All of the rings we have been considering (dagger algebra, fringe algebras, Robba rings) carry topologies, so it is natural to fit them into the framework of the previous section. Let us see how this is done.

We start with some simple observations.

- A dagger algebra with its fringe topology is an LF-space. This can be verified by noting that $K\left\langle x_{1}, \ldots, x_{n}\right\rangle^{\dagger}$ is an LF-space (as in Cr. Proposition 5.5]), then noting that a separated quotient of an LF-space is again an LF-space.

- For any dagger algebra $A$ and any finite set $T$, the rings $\mathcal{R}_{A, r}^{T}$ and $\mathcal{R}_{A}^{T,+}$ are Fréchet; hence $\mathcal{R}_{A}^{T}$ is an LF-space.

- For any dagger algebra $A$, the continuous map $A\langle x\rangle^{\dagger} \rightarrow \mathcal{R}_{A}$ carrying $x$ to $t^{-1}$ is strict with closed image, as is the map $\mathcal{R}_{A}^{+} \rightarrow \mathcal{R}_{A}$. Similarly, the map $K\left\langle x_{1}, \ldots, x_{n}\right\rangle^{\dagger} \rightarrow$ $K\left\langle x_{1}, \ldots, x_{n}, x_{1}^{-1}\right\rangle^{\dagger}$ is strict with closed image.

- For any affinoid algebra $A$ and any $f \in A^{\text {int }}$ whose reduction is not a zero divisor, the localization $A \rightarrow A\left\langle f^{-1}\right\rangle$ is an injective isometry, and hence is strict.

- If $A \rightarrow B$ is a strict morphism of reduced affinoid or dagger algebras, then the map $\mathcal{R}_{A} \rightarrow \mathcal{R}_{B}$ is also strict.

- Any morphism $R \rightarrow S$ satisfying one of the conditions of Proposition 3.2.6 or Remark 3.2.7 is strict with closed image: the topological vector space $S$ splits as the direct sum of $R$ with the kernel of the trace map.

Proposition 8.4.1. Let $A$ be an integral dagger algebra of $M W$-type, and suppose $f \in A$ satisfies $|f|_{\text {sup }, \widehat{A}}=1$. Then the map $A \rightarrow A\left\langle f^{-1}\right\rangle^{\dagger}$ is strict.

Proof. Since it is safe to pass from $K$ to a finite extension at any point in the argument, we will do so without further comment. Let $X$ be the special fibre of $A$, so that $X$ is smooth over $k$. We are trying to show that the passage to the localization of $A$ corresponding to the passage from $X$ to a particular basic open subset $U$ is strict; it suffices to show the same thing after passing to an even smaller $V$, or after passing from $X$ to a cover by open affines. 
Note that the claim is clear in case there is a strict injection $A \hookrightarrow \mathcal{R}_{B}^{+}$carrying $f$ to $t$, as we already know that $\mathcal{R}_{B}^{+} \rightarrow \mathcal{R}_{B}$ is a strict injection. This injection exists if and only if the completion of $X$ along $X \backslash U$ can be written as a fibration over $X \backslash U$; this is true locally if $X \backslash U$ is smooth, so we are done in that case.

In general, we can now cut out any smooth subvariety of $X$ by embedding it into a smooth divisor (we may have to pass to a cover of $X$ in order for the complement of that divisor to become a basic open). Now stratify $X \backslash U$ as follows. First enlarge $k$ if needed to ensure that each component of $X \backslash U$ is generically smooth. Then discard the smooth locus of each component and repeat until the process is forced to terminate by the noetherianness of $X$. We can now cut out the elements of the stratification one by one, from smallest to largest, so as to ultimately cut out all of $X \backslash U$ (and possibly more). This yields the claim.

We turn next to the topology of $(\sigma, \nabla)$-modules and their cohomology. The basic observation we need is the following, which follows directly from Proposition 3.1.4.

Proposition 8.4.2. For any reduced dagger algebra $A$, the map $d: \mathcal{R}_{A} \rightarrow \Omega_{\mathcal{R}_{A} / A}^{1}$ is strict with closed image.

From this simple fact we gain quite a lot of mileage.

Proposition 8.4.3. Let $A$ be an integral dagger algebra of $M W$-type, and let $M$ be $a(\sigma, \nabla)$ module over $\mathcal{R}_{A}$, relative to $A$. Then there exists a localization $A^{\prime}$ of $A$ such that $\nabla$ : $M \otimes_{\mathcal{R}_{A}} \mathcal{R}_{A^{\prime}} \rightarrow M \otimes_{\mathcal{R}_{A}} \Omega_{\mathcal{R}_{A^{\prime}} / A^{\prime}}^{1}$ is strict with closed image, and remains so after further localization.

Proof. Set notation as in Theorem 5.1.3. Then $M \otimes_{\mathcal{R}_{A}} \mathcal{R}^{\prime \prime} \rightarrow M \otimes_{\mathcal{R}_{A}} \Omega_{\mathcal{R}^{\prime \prime} / A}^{1}$ is strict by Proposition 8.4.2 plus a direct calculation (as in Lemma 7.1.1). By taking traces and invoking the criterion of Definition 8.3.4, we obtain the desired strictness. (We get stuck at $A^{\prime}$ because we cannot take a trace to pass from $A^{\prime}$ down to $A$.)

The following result has already been invoked via Lemma 7.5.1, but neither that result nor any of its consequences have been invoked in this chapter, so no vicious circle has been created.

Proposition 8.4.4. Let $A$ be an integral dagger algebra of $M W$-type, and let $M$ be a $(\sigma, \nabla)$ module over $A\langle x\rangle^{\dagger}$, relative to $A$. Then there exists a localization $A^{\prime}$ of $A$ such that $\nabla_{v}$ : $M \otimes_{A\langle x\rangle^{\dagger}} A^{\prime}\langle x\rangle^{\dagger} \rightarrow M \otimes_{A\langle x\rangle^{\dagger}} \Omega_{A^{\prime}\langle x\rangle^{\dagger} / A^{\prime}}$ is strict with closed image, and remains so after further localization.

Proof. Replace $A$ with a localization for which Theorem [7.3.3 and Proposition 8.4.3 hold for $M$. We first check that $\operatorname{im}\left(\nabla_{v}\right)$ is closed. Since the horizontal rows in (7.3.2.1) are strict exact, $\operatorname{im}\left(\nabla_{v}\right)$ is homeomorphic to the image of the composite map $M \rightarrow M \otimes \Omega_{\mathcal{R}_{A} / A}^{1}$, and it suffices to check that the latter is closed. Since $\nabla_{v}^{\text {loc }}$ is strict with closed image, it also suffices to check that $M+\operatorname{ker}\left(\nabla_{v}^{\text {loc }}\right)$ is closed in $M \otimes \mathcal{R}_{A}$, or that the image of $\operatorname{ker}\left(\nabla_{v}^{\text {loc }}\right)$ 
in $\left(M \otimes \mathcal{R}_{A}\right) / M$ is closed. But the latter image is finitely generated over $A$, hence always closed.

Since $\operatorname{im}\left(\nabla_{v}\right)$ is closed, $R^{1} f_{*} M$ is separated; after possibly localizing $A$ further, we can ensure that $R^{1} f_{*} M$ is finite free over $A$. Choosing free generators and lifting to $M \otimes_{A\langle x\rangle^{\dagger}}$ $\Omega_{A\langle x\rangle^{\dagger} / A}^{1}$, we see that $\operatorname{im}\left(\nabla_{v}\right)$ admits a topological complement and so is a direct summand. By [Cr, Proposition 3.5] (applicable since the source and target of $\nabla_{v}$ are LF-spaces), $\nabla_{v}$ is strict.

Proposition 8.4.5. Let $M$ be a $(\sigma, \nabla)$-module over a dagger algebra $A$, and suppose that the spaces $H^{i}(M)$ are finite-dimensional and separated for all $i$. Then all of the differentials $\nabla_{i}: M \otimes_{A} \Omega_{A / K}^{i} \rightarrow M \otimes_{A} \Omega_{A / K}^{i+1}$ are strict with closed image.

Proof. The map $f: M \otimes_{A} \Omega_{A / K}^{i} \rightarrow \operatorname{ker}\left(\nabla_{i+1}\right)$ has cokernel $H^{i+1}(M)$, which by hypothesis is finite-dimensional and separated, so the map is strict with closed image by [Cr, Corollary 3.6]. The map $\nabla$ factors as the composition of $f$ with the inclusion of a closed subspace, so it is also strict with closed image.

\subsection{Poincaré duality on affine space}

Having given an explicit description of the cohomology with compact supports of an overconvergent $F$-isocrystal on $\mathbb{A}^{n}$, we can now explicitly verify the nondegeneracy of the Poincaré pairing. We continue to appeal to $\mathrm{Cr}$ for topological details. Note that Hypothesis [8.1.1] and all subsequent definitions and notations are in force throughout this section.

Recall that $\mathcal{R}_{K}^{\left\{t_{1}, \ldots, t_{n}\right\},+}$ is a Fréchet space and observe that it is also Montel, by the same proof as [Cr, Corollary 5.3]. Then note that the natural map from $t_{1} \cdots t_{n} \mathcal{R}_{K}^{\left\{t_{1}, \ldots, t_{n}\right\},+}$ to $\mathcal{S}_{r}^{(n)} / \sum_{i} \mathcal{S}_{r}^{(n), i^{\prime}}$ is a homeomorphism, so the latter is also a Fréchet-Montel space.

The following basic result is proved in the same manner as [Cr. Proposition 5.5] (which is essentially the one-dimensional case).

Lemma 8.5.1. The pairing

$$
K\left\langle x_{1}, \ldots, x_{n}\right\rangle^{\dagger} \otimes_{K} \mathcal{S}_{r}^{(n)} / \sum_{i} \mathcal{S}_{r}^{(n), i^{\prime}} \rightarrow K
$$

taking $a \otimes b$ to the coefficient of $t_{1} \cdots t_{n}$ in $a b$ is perfect: that is, the induced map of each space to the strong dual of the other is an isomorphism of topological vector spaces.

Definition 8.5.2. Given a $(\sigma, \nabla)$-module $M$ on $K\left\langle x_{1}, \ldots, x_{n}\right\rangle^{\dagger}$, put

$$
C^{i}=\frac{M \otimes \Omega_{\mathcal{S}_{r}^{(n)} / K}^{i}}{\sum_{j=1}^{n} M \otimes \Omega_{\mathcal{S}_{r}^{(n), j^{\prime}} / K}^{i}},
$$

and put

$$
D^{i}=M^{\vee} \otimes \Omega_{K\left\langle x_{1}, \ldots, x_{n}\right\rangle^{\dagger} / K}^{i},
$$

topologized using the fringe topology of $K\left\langle x_{1}, \ldots, x_{n}\right\rangle^{\dagger}$. 
We quickly recall the pairing $[\cdot, \cdot]: C^{i} \otimes D^{n-i} \rightarrow K$ on the chain level that induces the Poincaré pairing described in Section 8.2. Given elements of $C^{i}$ and $D^{n-i}$ represented by $\mathbf{v} \in M \otimes \Omega_{\mathcal{S}_{r}^{(n)} / K}^{i}$ and $\mathbf{w} \in M^{\vee} \otimes \Omega_{K\left\langle x_{1}, \ldots, x_{n}\right\rangle^{\dagger} / K}^{n-i}$, pair $\mathbf{v}$ and $\mathbf{w}$ and apply the natural pairing $M \otimes M^{\vee} \rightarrow K$ to obtain an element of $\Omega_{\mathcal{S}_{r}^{(n)} / K}^{n}$. Then take the residue (the coefficient of $\left.\left(d t_{1} \wedge \cdots \wedge d t_{n}\right) /\left(t_{1} \cdots t_{n}\right)\right)$ and call the result $[\mathbf{v}, \mathbf{w}]$.

The pairing $[\cdot, \cdot]$ expresses each of $C^{i}$ and $D^{n-i}$ as the strong dual of the other by Lemma 8.5.1. It also has the property that $[\mathbf{v}, \nabla \mathbf{w}]=-[\nabla \mathbf{v}, \mathbf{w}]$ (by the Leibniz rule), and so induces a pairing on the homologies of the complexes $C$ and $D$. If $M$ corresponds to an overconvergent $F$-isocrystal $\mathcal{E}$ on $\mathbb{A}^{n}$, that induced pairing corresponds to the Poincaré pairing $H_{c, \text { rig }}^{n+i}\left(\mathbb{A}^{n}, \mathcal{E}\right) \otimes_{K} H_{\text {rig }}^{n-i}\left(\mathbb{A}^{n}, \mathcal{E}^{\vee}\right) \rightarrow K$.

Proposition 8.5.3. Let $\mathcal{E}$ be an overconvergent $F$-isocrystal on $\mathbb{A}^{n}$, and assume that $H_{\text {rig }}^{i}\left(\mathbb{A}^{n}, \mathcal{E}^{\vee}\right)$ is finite dimensional and separated for each $i$. Then the Poincaré pairing $H_{c, \text { rig }}^{n+i}\left(\mathbb{A}^{n}, \mathcal{E}\right) \otimes_{K}$ $H_{\text {rig }}^{n-i}\left(\mathbb{A}^{n}, \mathcal{E}^{\vee}\right) \rightarrow K$ is nondegenerate (i.e., each factor injects into the dual of the other).

Proof. By Proposition 8.4.5. $\nabla: D^{n-i} \rightarrow D^{n-i+1}$ is strict for each $i$; by Proposition 8.3.5 (applicable because each $D^{i}$ is Fréchet-Montel), $\nabla: C^{i} \rightarrow C^{i+1}$ is also strict for each $i$.

We first check that the Poincaré pairing is nondegenerate on the right. For any $\mathbf{v} \in$ $D^{n-i} \backslash \operatorname{im}\left(D^{n-i-1} \rightarrow D^{n-i}\right)$, we may use the fact that $\operatorname{im}\left(D^{n-i-1} \rightarrow D^{n-i}\right)$ is closed (by Proposition 8.4.5) to define a continuous linear map $\operatorname{im}\left(D^{n-i-1} \rightarrow D^{n-i}\right)+K \mathbf{v} \rightarrow K$ sending $\operatorname{im}\left(D^{n-i-1} \rightarrow D^{n-i}\right)$ to zero and $\mathbf{v}$ to 1 . By Proposition 8.3.6. this map extends to a continuous linear map $f: D^{n-i} \rightarrow K$, which corresponds by duality to some $\mathbf{w} \in C^{i}$. Now $[\nabla \mathbf{w}, \mathbf{x}]=-[\mathbf{w}, \nabla \mathbf{x}]=0$ for all $\mathbf{x} \in D^{n-i-1}$, so by perfectness of the residue pairing, $\nabla \mathbf{w}=0$. Thus $\mathbf{w}$ represents an element of $H_{c, \text { rig }}^{n+i}\left(\mathbb{A}^{n}, \mathcal{E}\right)$, which pairs with the given element of $H_{\text {rig }}^{n-i}\left(\mathbb{A}^{n}, \mathcal{E}^{\vee}\right)$ to a nonzero element of $K$. Hence the Poincaré pairing is nondegenerate on the right.

We next check that the Poincaré pairing is nondegenerate on the left. Given $\mathbf{w} \in C^{i}$ such that $[\mathbf{w}, \mathbf{v}]=0$ for any $\mathbf{v} \in D^{n-i}$ such that $\nabla \mathbf{v}=0$, we obtain from $\mathbf{w}$ a continuous linear map $f: D^{n-i} / \operatorname{ker}\left(D^{n-i} \rightarrow D^{n-i+1}\right) \rightarrow K$. Since $\nabla: D^{n-i} \rightarrow D^{n-i+1}$ is strict, the map $f$ induces a continuous linear map $g: \operatorname{im}\left(D^{n-i} \rightarrow D^{n-i+1}\right) \rightarrow K$. By Proposition 8.3.6. $g$ extends to a continuous linear map $h: D^{n-i+1} \rightarrow K$, which corresponds by duality to an element $\mathbf{x}$ of $C^{i-1}$. Now for any $\mathbf{v} \in D^{n-i}$,

$$
[\mathbf{w}+\nabla(\mathbf{x}), \mathbf{v}]=[\mathbf{w}, \mathbf{v}]-[\mathbf{x}, \nabla(\mathbf{v})]=f(\mathbf{v})-g(\nabla(\mathbf{v}))=0 .
$$

Thus $\mathbf{w}+\nabla(\mathbf{x})=0$ by perfectness of the residue pairing, so $\mathbf{w}$ vanishes in cohomology. Therefore the Poincaré pairing is nondegenerate on the left, completing the proof.

\subsection{Relative Poincaré duality}

We mention in passing that Poincaré duality also holds in the relative setting. Although we will not use this fact hereafter, it provides a useful tool for controlling the shape of the pushforwards; see for instance [Ke6]. 
Proposition 8.6.1. Let $A$ be an integral dagger algebra of $M W$-type, and let $f: A \rightarrow A\langle x\rangle^{\dagger}$ be the canonical inclusion. Let $M$ be $a(\sigma, \nabla)$-module over $A\langle x\rangle^{\dagger}$, and suppose $M$ and $M^{\vee}$ satisfy the conclusion of Theorem 7.3.3 without further localization. Then there are canonical perfect pairings

$$
\begin{array}{r}
R^{0} f_{*} M \otimes_{A} R^{2} f_{!} M^{\vee} \rightarrow A \\
R_{\mathrm{loc}}^{i} f_{*} M \otimes_{A} R_{\mathrm{loc}}^{1-i} f_{*} M^{\vee} \rightarrow A \\
R^{1} f_{!} M \otimes_{A} R^{1} f_{*} M^{\vee} \rightarrow A \\
R_{p}^{1} f_{*} M \otimes_{A} R_{p}^{1} f_{*} M^{\vee} \rightarrow A .
\end{array}
$$

Again, these pairings are induced by the natural pairing $M \otimes_{A} M^{\vee} \rightarrow A$ and the residue map $\Omega_{\mathcal{R}_{A} / A}^{1} \rightarrow A$ sending $\omega$ to its coefficient of $d t / t$. The fourth pairing exists because of a compatibility between the others: given $\alpha \in R_{\text {loc }}^{0} f_{*} M$ mapping to $\beta \in R^{1} f_{!} M$ and $\gamma \in R^{1} f_{*} M^{\vee}$ mapping to $\delta \in R_{\mathrm{loc}}^{1} f_{*} M^{\vee}$, then $[\alpha, \delta]=[\beta, \gamma]$.

Proof. By flat base change to the generic point (Remark 7.5.4), Poincaré duality on $\mathbb{A}^{1}$ (Proposition 8.5.3. whose finite-dimensionality and separatedness hypotheses are satisfied as in the proof of [Cr, Theorem 9.5]), and the finite dimensionality of $R^{0} f_{*} M$ and $R^{2} f_{!} M^{\vee}$ (Theorem [7.3.3), the map

$$
R^{0} f_{*} M \rightarrow\left(R^{2} f_{!} M^{\vee}\right)^{\vee}
$$

becomes an isomorphism upon tensoring with the completion of Frac $A$ for the affinoid topology. However, the kernel and cokernel of this map are finitely generated $A$-modules with connection, so by Lemma 3.3.4 they are locally free, necessarily of rank zero. Thus in fact they both vanish, and the map is an isomorphism, that is, the first pairing above is perfect. The same argument applies to $R^{1} f_{*} M \rightarrow\left(R^{1} f_{!} M^{\vee}\right)^{\vee}$; the perfectness of the other two pairings follows from the five lemma.

\section{Finite dimensionality: the main results}

In this chapter, we prove the results enunciated in Chapter 1. While up to now we have made all our calculations of rigid cohomology on smooth affine schemes, where the MonskyWashnitzer description can be used, at this point we wish to prove more general results. We will thus use the formalism of rigid cohomology in full strength, reducing everything to consideration of smooth affine schemes. See Chapter $\square$ for more details.

\subsection{Cohomology without supports: the smooth case}

In this section, we prove the restriction of Theorem 1.2.1 to the case where $X$ is smooth.

Proposition 9.1.1. Let $\mathcal{E}$ be an overconvergent $F$-isocrystal on a smooth scheme $X$ over a field $k$ of characteristic $p>0$. Then the rigid cohomology spaces $H_{\mathrm{rig}}^{i}(X / K, \mathcal{E})$ are finite dimensional for all $i$. 
The gross outline of the proof follows that given by Berthelot [Be5] in the constant coefficient case, that given by Tsuzuki [T3, Theorem 6.1.1] in the unit-root case, and the proposed proof of Shiho [Sh] in the general case. However, we simplify matters using the following geometric fact, for whose proof see [Ke4]. (A proof in case $k$ is infinite and perfect can be found in [Ke3].)

Proposition 9.1.2. Let $X$ be a separated, geometrically reduced, finite type $k$-scheme of dimension $n$, for $k$ a field of characteristic $p>0$, and let $x$ be a smooth geometric point of $X$. Then $X$ contains an open dense affine subscheme containing $x$ and admitting a finite étale morphism to affine n-space.

This result allows us to "go down" to simpler spaces (namely affine space). By contrast, in [Be5], [T3], and [Sh] (and Section 9.2, where we invoke cohomological descent), one simplifies spaces by "going up" using de Jong's alterations theorem.

Proof of Proposition 9.1.1. We prove the following two assertions by induction on $n$ :

$(\text { a })_{n} H_{\text {rig }}^{i}(X / K, \mathcal{E})$ is finite dimensional for any $X$ smooth over $k$ of dimension at most $n$, and any overconvergent $F$-isocrystal $\mathcal{E}$ on $X$;

(b) $H_{Z, \text { rig }}^{i}(X / K, \mathcal{E})$ is finite dimensional for any closed immersion $Z \hookrightarrow X$ of a geometrically reduced scheme $Z$ of finite type over $k$ of dimension at most $n$ into a scheme $X$ smooth over $k$, and for any overconvergent $F$-isocrystal $\mathcal{E}$ on $X$.

For starters, $(\mathrm{a})_{n}$ is trivial for $n=0$ and $(\mathrm{b})_{n}$ is vacuous for $n=-1$. Now $(\mathrm{b})_{n-1}$ and $(\mathrm{a})_{n}$ together imply $(\mathrm{b})_{n}$ as follows. (The argument is copied essentially verbatim from Sh, Theorem 3.1.9].) First suppose that Hypothesis 4.4.1 is satisfied; that is, $Z \hookrightarrow X$ is a smooth pair, with $\operatorname{dim} Z \leq n$, admitting a lifting $\mathcal{Z} \hookrightarrow \mathcal{X}$ over $\mathcal{O}$, such that $\mathcal{Z}, \mathcal{X}$ are smooth over $\mathcal{O}$ and there exists an étale morphism $\mathcal{X} \rightarrow \operatorname{Spec} \mathcal{O}\left[x_{1}, \ldots, x_{d}\right]$ for some integer $d$, such that $\mathcal{Z}$ maps to the vanishing locus of $d-n$ of the $x_{i}$. Then we may apply Tsuzuki's construction of the Gysin isomorphism [T3, Theorem 4.1.1] to conclude that $H_{Z \text {,rig }}^{i}(X / K, \mathcal{E}) \cong H_{\text {rig }}^{i-2 c}\left(Z / K,\left.\mathcal{E}\right|_{Z}\right)$, where $c=\operatorname{dim} X-\operatorname{dim} Z$; by (a) $)_{n}$, the latter is finite dimensional.

To handle the general case, we use the excision exact sequence (4.4.0.1), which we recall for convenience:

$$
\cdots \rightarrow H_{T / K, \text { rig }}^{i}(X, \mathcal{E}) \rightarrow H_{Z / K, \text { rig }}^{i}(X, \mathcal{E}) \rightarrow H_{Z \backslash T, \text { rig }}^{i}(X \backslash T / K, \mathcal{E}) \rightarrow \cdots,
$$

Suppose $Z$ is any geometrically reduced subscheme of a smooth $k$-scheme $X$ with $\operatorname{dim} Z \leq n$. Then we can find some closed subscheme $T$ with $\operatorname{dim} T \leq n-1$ such that Hypothesis 4.4.1 holds for the pair $Z \backslash T \hookrightarrow X \backslash T$. Thus $H_{Z \backslash T \text {,rig }}^{i}(X \backslash T / K, \mathcal{E})$ is finite dimensional; by $(\mathrm{b})_{n-1}, H_{T, \text { rig }}^{i}(X / K, \mathcal{E})$ is also finite dimensional. Hence by (4.4.0.1), $H_{Z \text {,rig }}^{i}(X / K, \mathcal{E})$ is finite dimensional; we thus conclude that $(\mathrm{b})_{n-1}$ and $(\mathrm{a})_{n}$ imply $(\mathrm{b})_{n}$.

Now we prove that $(\mathrm{a})_{n-1}$ and $(\mathrm{b})_{n-1}$ imply $(\mathrm{a})_{n}$. To prove finite dimensionality of $H_{\text {rig }}^{i}(X / K, \mathcal{E})$ for an overconvergent $F$-isocrystal $\mathcal{E}$ on $X$, we may make the following replacements without loss of generality: 
(i) replace $K$ by a finite extension;

(ii) replace $(X, \mathcal{E})$ by $\left(U, j^{*} \mathcal{E}\right)$ for any open immersion $j: U \hookrightarrow X$ with dense image;

(iii) replace $(X, \mathcal{E})$, if $X$ is affine, by $\left(Y, f_{*} \mathcal{E}\right)$ for any finite étale morphism $f: X \rightarrow Y$.

The reduction (i) follows from Proposition 3.4.3 (or by finite flat base change, as in the next section). The reduction (ii) follows from $(\mathrm{b})_{n-1}$ and the excision sequence (4.4.0.1) with $T$ the reduced subscheme of $X \backslash U$ and $Z=X$ (and applying (i) if necessary so that $T$ becomes geometrically reduced). The reduction (iii) follows from Proposition 3.4.5, as the dimensions of the cohomology spaces do not change.

For starters, (ii) means we can replace $X$ with an open dense affine subset $V$. By Proposition 9.1.2 there exists an open immersion $j: U \hookrightarrow V$ with dense image such that $U$ admits a finite étale map $f: U \rightarrow \mathbb{A}^{n}$. By (ii) and (iii), to prove finiteness for $(X, \mathcal{E})$, it suffices to prove finiteness for $\left(\mathbb{A}^{n}, f_{*} j^{*} \mathcal{E}\right)$. Now write $\mathbb{A}^{n}$ as a product $\mathbb{A}^{1} \times \mathbb{A}^{n-1}$. By Theorem 7.3 .3 , the kernel $\mathcal{F}_{0}$ and cokernel $\mathcal{F}_{1}$ of the vertical connection $\nabla_{v}$ become overconvergent $F$ isocrystals on some open subset $W$ of $\mathbb{A}^{n-1}$.

By $(\text { a })_{n-1}, H_{\text {rig }}^{i}\left(W / K, \mathcal{F}_{0}\right)$ and $H_{\text {rig }}^{i}\left(W / K, \mathcal{F}_{1}\right)$ are finite dimensional for all $i$; by Proposition [7.4.1. we conclude that $H_{\text {rig }}^{i}\left(\mathbb{A}^{1} \times W / K, f_{*} j^{*} \mathcal{E}\right)$ is finite dimensional for all $i$. Thus $H_{\text {rig }}^{i}(X / K, \mathcal{E})$ is finite dimensional for all $i$, and the induction is complete.

\subsection{Cohomology without supports: the general case}

We now proceed to the proof of Theorem 1.2.1 in general, relaxing the hypothesis that $X$ is smooth. We will obtain the theorem from Proposition 9.1.1 using the technique of cohomological descent, developed in rigid cohomology by Chiarellotto and Tsuzuki [CT], T5].

Proof of Theorem 1.2.1. First recall a consequence of the finite flat base change theorem [CT, Theorem 11.8.1] (or see [Be5, Proposition 1.8] for the constant coefficient case): if $K^{\prime}$ is a finite extension of $K$ with residue field $k^{\prime}$, then for any separated scheme $X$ of finite type over $k$ and any overconvergent $F$-isocrystal $\mathcal{E}$ on $X$, we have

$$
H_{\text {rig }}^{i}(X / K, \mathcal{E}) \otimes_{K} K^{\prime} \cong H_{\text {rig }}^{i}\left(X \times_{k} k^{\prime} / K^{\prime}, \mathcal{E}\right) .
$$

We now imitate the argument of [T5, Theorem 5.1.1] using nonconstant coefficients. Let $X$ be a separated scheme of finite type over $k$, and let $\mathcal{E}$ be an overconvergent $F$-isocrystal over $X$. The alterations theorem of de Jong [dJ1, Theorem 4.1] and the construction of [5. Section 4] produce a proper hypercovering $f . Y . \rightarrow X$ over Spec $k$ and an increasing sequence $k_{0} \subseteq k_{1} \subseteq \cdots$ of finite purely inseparable extensions of $k$, such that $Y_{n}$ is smooth over $k_{n}$ for each $n$. Choose an increasing sequence $K_{0} \subseteq K_{1} \subseteq \cdots$ of finite extensions of $K$ such that $K_{n}$ has residue field $k_{n}$ for each $n$. Then $Y_{n} \times_{k} k_{n}$ may not be smooth over $k_{n}$, but the canonical immersion $Y_{n} \hookrightarrow Y_{n} \times_{k} k_{n}$ over Spec $k_{n}$ is a nilpotent immersion, so it induces an isomorphism $H_{\text {rig }}^{i}\left(Y_{n} \times_{k} k_{n} / K_{n}, f_{n}^{*} \mathcal{E}\right) \cong H_{\text {rig }}^{i}\left(Y_{n} / K_{n}, f_{n}^{*} \mathcal{E}\right)$. By Proposition 9.1.1, 
these spaces are finite dimensional over $K_{n}$, as then is $H_{\text {rig }}^{i}\left(Y_{n} / K, f_{n}^{*} \mathcal{E}\right)$ over $K$ by the finite flat base change theorem described above. Now [T5, Theorem 4.5.1] produces a spectral sequence in which

$$
E_{1}^{q r}=H_{\text {rig }}^{r}\left(Y_{q} / K, f_{q}^{*} \mathcal{E}\right) \Rightarrow H_{\text {rig }}^{q+r}(X / K, \mathcal{E}) .
$$

Since all terms of $E_{1}$ are finite dimensional over $K$, the spaces $H_{\text {rig }}^{i}(X / K, \mathcal{E})$ are as well.

Remark 9.2.1. By excision (4.4.0.1), it also follows that $H_{Z, \text { rig }}^{i}(X, \mathcal{E})$ is finite dimensional for any closed subscheme $Z$ of $X$, without any smoothness hypothesis; one also obtains the base change property.

Remark 9.2.2. We believe one can also carry out the above reduction of Theorem 1.2 .1 to Proposition 9.1.1 using the techniques used by Grosse-Klönne GK2 in the constant coefficient case. (Namely, one should locally embed the nonsmooth scheme into a smooth scheme and carefully control the cohomology of some ensuing dagger spaces.) This belief amounts to the claim that one can repeat the entire argument of GK2 with nonconstant coefficients (coherent modules with connection on dagger spaces), using Proposition 9.1.1 in lieu of $[\mathrm{Be} 5]$. We have not verified this claim.

\subsection{Poincaré duality and cohomology with compact supports}

We now consider rigid cohomology with compact supports. In this case, the relevant excision sequence, for $Z$ a closed subscheme of $X$ and $U=X \backslash Z$, is (4.4.1.1):

$$
\cdots \rightarrow H_{c, \text { rig }}^{i}(U, \mathcal{E}) \rightarrow H_{c, \text { rig }}^{i}(X, \mathcal{E}) \rightarrow H_{c, \text { rig }}^{i}(Z, \mathcal{E}) \rightarrow \cdots
$$

Since all three types of terms in the sequence have the same form, the proof of finite dimensionality is somewhat simpler in this case, and in particular there is no need to separately handle the smooth case, use a Gysin isomorphism or invoke any descent results. All we need to do is first check Poincaré duality, then use Theorem 1.2.1.

As in the case of cohomology without supports, we have a base change isomorphism

$$
H_{c, \text { rig }}^{i}(X / K, \mathcal{E}) \otimes_{K} K^{\prime} \cong H_{c, \text { rig }}^{i}\left(X \times_{k} k^{\prime} / K^{\prime}, \mathcal{E}\right)
$$

for $K^{\prime}$ a finite extension of $K$ with residue field $k^{\prime}$, and the proof is analogous; again, see [Be5, Proposition 1.8].

Before proving anything about Poincaré duality, we need to clarify the nature of its target space. We do this by starting with some basic computations; these duplicate [Be6. Propositions 1.1 and 1.5], but we prefer to reproduce them in our context (in particular, without comparison to crystalline cohomology).

Lemma 9.3.1. Let $X$ be a separated, finite type $k$-scheme of dimension $n$. Then $H_{c, \text { rig }}^{d}(X / K)=$ 0 for $d>2 n$. 
Proof. We proceed by induction on $n$. We may ensure that $X$ is geometrically reduced by making a base change. By excision (4.4.1.1), we may replace $X$ by an open dense subset $U$; in particular, we choose $U$ by virtue of Proposition 9.1.2 so that there exists a finite étale map $f: U \rightarrow \mathbb{A}^{n}$. Let $\mathcal{E}$ be the pushforward along $f$ of the trivial $F$-isocrystal on $U$; then $H_{c, \text { rig }}^{d}(U / K) \cong H_{c \text {,rig }}^{d}\left(\mathbb{A}^{n} / K, \mathcal{E}\right)$ by Proposition 4.4.2. However, the latter manifestly vanishes from the description given in Section 8.1. This yields the desired result.

Again, let $X$ be a separated, finite type $k$-scheme of pure dimension $n$. Using Lemma 9.3.1, one may construct a canonical trace morphism

$$
\operatorname{Tr}: H_{c, \text { rig }}^{2 n}(X / K) \rightarrow H_{c, \text { rig }}^{0}(\operatorname{Spec} k / K) \cong K
$$

as in [Be6, 1.2]. That construction completes the description of the Poincaré pairing begun in Section 8.2 and now we may begin to consider its perfectness.

Lemma 9.3.2. Let $X$ be a separated, finite type, geometrically irreducible $k$-scheme of dimension $n$. Then $H_{c, \text { rig }}^{2 n}(X / K)$ is one-dimensional over $K$.

Note that this assertion does not include the fact that the trace map on $X$ is nonzero; that will follow from the general proof of Poincaré duality.

Proof. Again, we can reduce to the case where $X$ is geometrically reduced by a base change. By excision (4.4.1.1) and Lemma 9.3.1, we may again replace $X$ by an open dense subset admitting a finite étale map to $\mathbb{A}^{n}$. Viewing $\mathbb{A}^{n}$ as a family of $\mathbb{A}^{1}$ 's with base $\mathbb{A}^{n-1}$, we may shrink the base so that pushforwards exist as in Theorem [7.3.3, and the conclusion of Proposition 8.4.3 holds. Shrink the base and push forward again to convert into another $\mathbb{A}^{n-1}$, then view $\mathbb{A}^{n-1}$ as $\mathbb{A}^{1} \times \mathbb{A}^{n-2}$, shrink $\mathbb{A}^{n-2}$ until we can push forward again, et cetera.

At the end of this process, we obtain an open dense subset $U$ of $X$ admitting a finite étale map $f: U \rightarrow \mathbb{A}^{n}$. Retain notation as in Lemma 9.3.1 and topologize the spaces $H^{i}\left(\mathbb{A}^{n} / K, \mathcal{E}\right)$ by identifying them with the cohomology of the corresponding $(\sigma, \nabla)$-module $M$ over $K\left\langle x_{1}, \ldots, x_{n}\right\rangle^{\dagger}$. In the argument (and notation) of Proposition 7.4.1, the differentials $d_{0}$ are strict by Proposition 8.4.4, so the cohomology of $H^{i}(M)$ is correctly computed as a topological space by $E_{1}$. Suppose that the hypothesis of Proposition 8.4.5 is satisfied for $P$ and $Q$; then by Proposition 8.4.5, the differentials $d_{1}$ are strict, so the cohomology of $H^{i}(M)$ is correctly computed by $E_{2}$. That is, the exact sequence of Proposition [7.4.1] is strict, so the $H^{i}(M)$ are finite dimensional and separated. Applying the same argument to $P$ and $Q$, we recurse down the dimensions to ultimately conclude that the finite dimensionality and separatedness conditions in Proposition 8.5.3 are satisfied.

We now may invoke Poincaré duality on $\mathbb{A}^{n}$ (Proposition 8.5.3) to ensure that $H_{c, \text { rig }}^{2 n}\left(\mathbb{A}^{n} / K, \mathcal{E}\right)$ is canonically isomorphic to the dual of $H_{\text {rig }}^{0}\left(\mathbb{A}^{n} / K, \mathcal{E}^{\vee}\right)$, which in turn by Proposition 3.4.5 is canonically isomorphic to $H_{\text {rig }}^{0}(U / K)$. (Here we are using the fact that $\mathcal{E}^{\vee} \cong \mathcal{E}$ via the trace from $U$ to $\mathbb{A}^{n}$.) But the latter is canonically isomorphic to $H_{\text {rig }}^{0}(\operatorname{Spec} k / K)$ by [MW] Theorem 7.1], whence the desired result. 
For details on the functoriality of the Poincaré pairing, see [T3, Section 6.2]. One fact we need is that for $X$ smooth, the excision sequences (4.4.0.1) and 4.4.1.1) fit into a commutative diagram

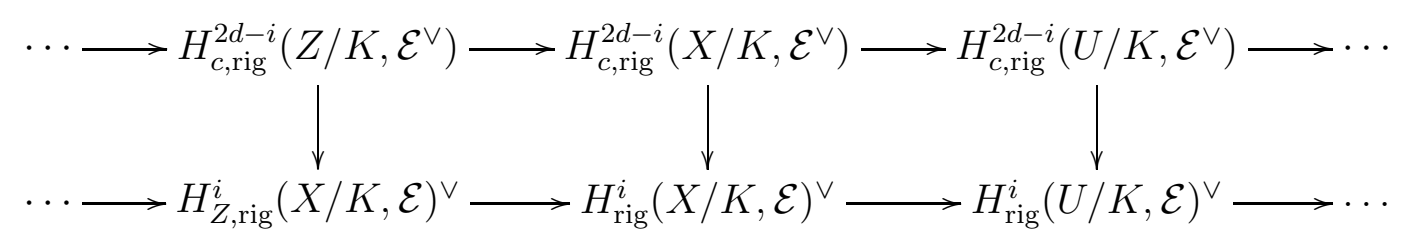

in which the vertical arrows are induced by the pairing (1.2.3.1). Note that the spaces being dualized in the bottom row are already known to be finite dimensional by Theorem 1.2.1, so there is no ambiguity about what sort of duals to take.

We exploit this diagram via an inductive procedure similar to that of [T3, Theorem 6.2.5].

Proof of Theorem 1.2.3. We prove the following assertions by simultaneous induction on $n$, where $(\mathrm{a})_{0}$ is straightforward.

$(\mathrm{a})_{n}$ The pairing (1.2.3.1) is perfect whenever $Z=X$ and $\operatorname{dim} X \leq n$.

(b) ${ }_{n}$ The pairing (1.2.3.1) is perfect whenever $\operatorname{dim} Z \leq n$.

We first observe that $(\mathrm{b})_{n-1}$ and $(\mathrm{a})_{n}$ imply $(\mathrm{b})_{n}$, by the same argument as in the proof of Theorem 1.2.1, only this time using the commutative diagram (9.3.2.1) in place of the excision sequence (4.4.0.1).

We next establish that $(\mathrm{b})_{n-1}$ implies $(\mathrm{a})_{n}$. Given $X$ and $\mathcal{E}$, we may choose an open dense subscheme $U$ of $X$ admits a finite étale morphism $f: U \rightarrow \mathbb{A}^{n}$. Taking $Z=X \backslash U$, considering the excision diagram (9.3.2.1), and applying the induction hypothesis to the left vertical arrow, we see that to prove perfectness of the pairing for $X$, it suffices to check it for $U$. To prove that, it in turn suffices by Proposition 4.4.2 to check perfectness for the isocrystal $f_{*} \mathcal{E}$ and its dual on $\mathbb{A}^{n}$.

As in the proof of Lemma 9.3.2, we can arrange the map $f$ so that the spaces $H_{\text {rig }}^{i}\left(\mathbb{A}^{n} / K, f_{*} \mathcal{E}\right)$ are known to be finite dimensional and separated. We may thus apply Proposition 8.5.3, the nondegeneracy of the Poincaré pairing implies first that the spaces $H_{c, \text { rig }}^{2 n-i}\left(\mathbb{A}^{n} / K, f_{*} \mathcal{E}^{\vee}\right)$ are finite dimensional, then that the pairing is in fact perfect. Hence $(\mathrm{b})_{n-1}$ implies $(\mathrm{a})_{n}$, completing the proof.

We now prove finite dimensionality of rigid cohomology with compact supports.

Proof of Theorem 1.2.2. Again, we induct on the dimension of $X$, the case of dimension -1 being vacuous. After replacing $k$ by a finite extension and enlarging $K$ appropriately, we may replace $X$ by its reduced subscheme and assume that the latter is irreducible and geometrically reduced. Then the complement of the singular locus $Z$ of $X$ is an open dense subscheme of $X$. The cohomology spaces $H_{c, \text { rig }}^{i}(Z / K, \mathcal{E})$ are finite dimensional by the induction hypothesis, while the cohomology spaces $H_{c \text {,rig }}^{i}(X \backslash Z / K, \mathcal{E})$ are finite dimensional by Theorems 1.2 .1 and Theorem 1.2 .3 . Thus $H_{c, \text { rig }}^{i}(X / K, \mathcal{E})$ is finite dimensional by the exactness of the excision sequence (4.4.1.1). 


\subsection{The Künneth decomposition}

This time, we defer to [T3, Section 6.3] for the construction and functoriality of the Künneth morphisms (1.2.4.1), (1.2.4.2) and concentrate on showing that these are isomorphisms, following [Be6, Théorème 3.2] and [T3, Theorem 6.3.6].

Proof of Theorem 1.2.4. We first establish that (1.2.4.2) is an isomorphism, by proving the following assertions by simultaneous induction on $n$.

$(\mathrm{a})_{n}$ The morphism (1.2.4.2) is an isomorphism whenever the $X_{i}$ are smooth, $Z_{i}=X_{i}$ $(i=1,2)$ and $\operatorname{dim} X \leq n$.

(b) ${ }_{n}$ The morphism (1.2.4.2) is an isomorphism whenever the $X_{i}$ are smooth and $\operatorname{dim} Z \leq n$.

To begin with, $(\mathrm{b})_{-1}$ is vacuous and $(\mathrm{a})_{0}$ is trivial. To show that $(\mathrm{b})_{n-1}$ and $(\mathrm{a})_{n}$ imply $(\mathrm{b})_{n}$, it is easiest to first prove $(\mathrm{b})_{n}$ assuming $Z_{2}=X_{2}$. In that case, one can "tensor" the excision exact sequence on $X_{1}$ with the cohomology of $X_{2}$ and map to $X$, then argue in the resulting diagram as in Proposition 9.1.1. This is a bit painful to write out in our notation; it is better put in the language of derived functors and triangulated categories (as in [Be6, 3.1]) by saying that we are tensoring the distinguished triangle

$$
\mathbb{R} \Gamma_{T_{1}, \mathrm{rig}}\left(X_{1} / K, \mathcal{E}_{1}\right) \rightarrow \mathbb{R} \Gamma_{Z_{1}, \mathrm{rig}}\left(X_{1} / K, \mathcal{E}_{1}\right) \rightarrow \mathbb{R} \Gamma_{Z_{1} \backslash T_{1}, \mathrm{rig}}\left(X_{1} \backslash T_{1} / K, \mathcal{E}_{1}\right) \stackrel{+1}{\rightarrow}
$$

with $\mathbb{R} \Gamma_{Z_{2} \text {,rig }}\left(X_{2} / K, \mathcal{E}_{2}\right)$, and so on.

To deduce that (a) $)_{n-1}$ and (b) $)_{n-1}$ imply (a) $)_{n}$, use Proposition 9.1.2 to choose open dense subschemes $U_{1}, U_{2}$ of $X_{1}, X_{2}$ which admit finite étale morphisms to affine spaces. By applying excision (as in the previous paragraph), (b) $)_{n-1}$ and Proposition 3.4.5, we may reduce to the case where $X_{1}$ is an affine space, $X_{2}=V \times \mathbb{A}^{1}$, and $R^{i} f_{*} \mathcal{E}_{2}$ is an overconvergent $F$-isocrystal on $V$ (for $f$ the projection of $V \times \mathbb{A}^{1}$ onto $V$ ). Let $g$ be the projection of $X_{1} \times X_{2}$ onto $X_{1} \times V$, Let $\operatorname{pr}_{1}: X_{1} \times V \rightarrow X_{1}$ and $\operatorname{pr}_{2}: X_{1} \times V \rightarrow V$ denote the obvious projections, and write $\mathcal{F} \otimes \mathcal{G}$ for the external product $\operatorname{pr}_{1}^{*} \mathcal{F} \otimes \operatorname{pr}_{2}^{*} \mathcal{G}$. We then have a commuting diagram

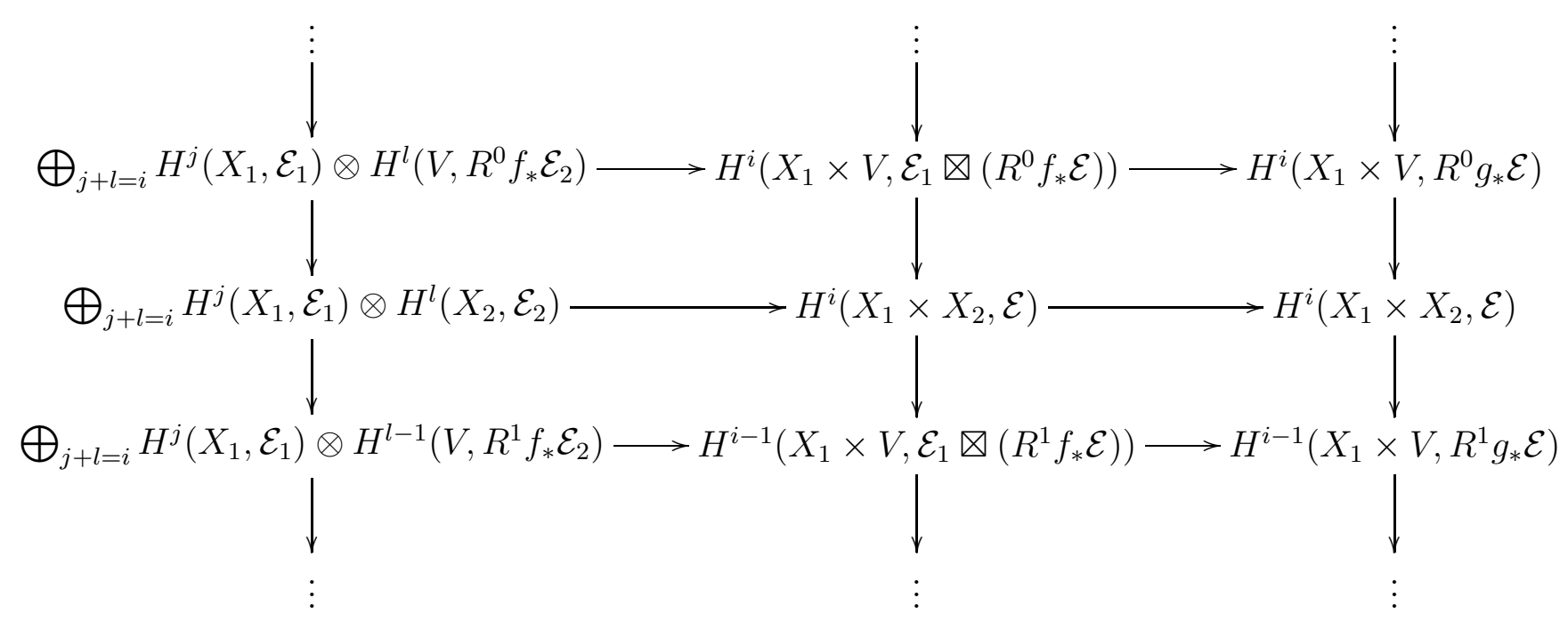


as follows. (We dropped " $K$ " and "rig" from the notation to reduce clutter.) The left and right vertical columns are long exact sequences obtained from Proposition [7.4.1. The middle column and its arrows to the right column are constructed from the isomorphisms of Proposition 7.6.2 (the middle horizontal arrow being the identity map). The arrows between the left and middle columns are the Künneth morphisms; of these, the top and bottom arrows are isomorphisms by $(\mathrm{a})_{n-1}$. Since this is true all the way down the long exact sequences, we conclude that the middle arrow is also an isomorphism by the five lemma. This yields $(\mathrm{a})_{n}$, completing the induction and proving that (1.2.4.2) is an isomorphism.

We now establish that (1.2.4.1) is always an isomorphism by induction on the dimension of $X$. Given $X$, let $Z$ be the singular locus of $X$ and put $U=X \backslash Z$. By excision as above and the induction hypothesis, it suffices to prove the Künneth isomorphism on $U$, but this follows from Poincaré duality (Theorem 1.2.3) together with the fact that (1.2.4.2) is always an isomorphism.

\section{References}

[An] Y. André, Filtrations de type Hasse-Arf et monodromie p-adique, Invent. Math. 148 (2002), 285-317.

[Ar] A. Arabia, Relèvements des algèbres lisses et de leurs morphismes, Comment. Math. Helv. 76 (2001), 607-639.

[Be1] P. Berthelot, Cohomologie cristalline des schémas de caractéristique $p>0$, Springer Lect. Notes in Math. 407 (1974).

[Be2] P. Berthelot, Géométrie rigide et cohomologie des variétés algebriques de caractéristique $p$, in Introductions aux cohomologies p-adiques (Luminy, 1984), Mém. Soc. Math. France 23 (1986), 7-32.

[Be3] P. Berthelot, Cohomologie rigide et théorie des $\mathcal{D}$-modules, in $p$-adic analysis (Trento, 1989), Springer Lect. Notes in Math. 1454 (1990), 80-124.

[Be4] P. Berthelot, Cohomologie rigide et cohomologie rigide à supports propres, Première partie (version provisoire 1991), Prépublication IRMAR 96-03, Université de Rennes (1996).

[Be5] P. Berthelot, Finitude et pureté cohomologique en cohomologie rigide (with an appendix in English by A.J. de Jong), Invent. Math. 128 (1997), 329-377.

[Be6] P. Berthelot, Dualité de Poincaré et formule de Künneth en cohomologie rigide, C.R. Acad. Sci. Paris 325 (1997), 493-498.

[Be7] P. Berthelot, Introduction à la théorie arithmétique des $\mathcal{D}$-modules, in Cohomologies p-adiques et applications arithmétiques, II, Astérisque 279 (2002), 1-80. 
[BO] P. Berthelot and A. Ogus, Notes on crystalline cohomology, Princeton University Press (Princeton, N.J.), 1978.

[BGR] S. Bosch, U. Güntzer, and R. Remmert, Non-Archimedean analysis, Grundlehren der math. Wiss. 261, Springer-Verlag (Berlin), 1984.

[BT] R. Bott and L. Tu, Differential forms in algebraic topology, GTM 82, Springer-Verlag (New York), 1982.

[Ca] D. Caro, D-modules arithmétiques surholonomes, preprint available at http://www.maths.dur.ac.uk/ dma1dc.

[CT] B. Chiarellotto and N. Tsuzuki, Cohomological descent of rigid cohomology for étale coverings, Rend. Sem. Mat. Univ. Padova 109 (2003), 63-215.

[CM1] G. Christol and Z. Mebkhout, Sur le théorème de l'indice des équations différentielles p-adiques. III, Ann. of Math. 151 (2000), 385-457.

[CM2] G. Christol and Z. Mebkhout, Sur le théorème de l'indice des équations différentielles p-adiques. IV, Invent. Math. 143 (2001), 629-672.

[Cr] R. Crew, Finiteness theorems for the cohomology of an overconvergent isocrystal on a curve, Ann. Sci. Éc. Norm. Sup. 31 (1998), 717-763.

[dJ1] A.J. de Jong, Smoothness, semi-stability and alterations, Publ. Math. IHÉS 83 (1996), 51-93.

[dJ2] A.J. de Jong, Barsotti-Tate groups and crystals, Proceedings of the ICM, Volume II (Berlin, 1998), Doc. Math. Extra Vol. II (1998), 259-265.

[dJ3] A.J. de Jong, Homomorphisms of Barsotti-Tate groups and crystals in positive characteristic, Invent. Math. 134 (1998), 301-333.

[De] P. Deligne, La conjecture de Weil. II, Publ. Math. IHÉS 52 (1980), 137-252.

[E] D. Eisenbud, Commutative algebra with a view toward algebraic geometry, GTM 150, Springer-Verlag (New York), 1995.

[F] W. Fulton, A note on weakly complete algebras, Bull. Amer. Math. Soc. 75 (1969), 591-593.

[Gr] B. Gray, Homotopy theory: an introduction to algebraic topology, Academic Press (New York), 1975.

[GK1] E. Grosse-Klönne, Rigid analytic spaces with overconvergent structure sheaf, J. reine angew. Math. 519 (2000), 73-95. 
[GK2] E. Grosse-Klönne, Finiteness of de Rham cohomology in rigid analysis, Duke Math. J. 113 (2002), 57-91.

[I] L. Illusie, Complexe de de Rham-Witt et cohomologie cristalline, Ann. Sci. Éc. Norm. Sup. 12 (1979), 501-661.

[Ke1] K.S. Kedlaya, A p-adic local monodromy theorem, Ann. Math. 160 (2004), 93-184.

[Ke2] K.S. Kedlaya, Semistable reduction for overconvergent $F$-isocrystals on a curve, Math. Res. Lett. 10 (2003), 151-159.

[Ke3] K.S. Kedlaya, Étale covers of affine spaces in positive characteristic, C.R. Acad. Sci. Paris 335 (2002), 921-926.

[Ke4] K.S. Kedlaya, More étale covers of affine spaces in positive characteristic, J. Alg. Geom. 14 (2005), 187-192.

[Ke5] K.S. Kedlaya, Local monodromy of $p$-adic differential equations: an overview, Int. J. Number Theory 1 (2005), 109-154.

[Ke6] K.S. Kedlaya, Fourier transforms and p-adic "Weil II", arXiv: math.NT/0210149 (version of 20 Jul 2005).

[Ke7] K.S. Kedlaya, Slope filtrations revisited, arXiv: math.NT/0504204 (version of 25 Oct 2005); to appear in Doc. Math.

[Ke8] K.S. Kedlaya, Semistable reduction for overconvergent $F$-isocrystals, I: Unipotence and logarithmic extensions, arXiv: math.NT/0405069 (version of 24 Jul 2005).

[Ki] R. Kiehl, Theorem A und Theorem B in der nichtarchimedischen Funktionentheorie, Invent. Math. 2 (1967), 256-273.

[La] M. Lazard, Les zéros des fonctions analytiques d'une variable sur un corps valué complet, Publ. Math. IHÉS 14 (1962), 47-75.

[Lu] S. Lubkin, Generalization of $p$-adic cohomology: bounded Witt vectors. A canonical lifting of a variety in characteristic $p \neq 0$ back to characteristic zero, Comp. Math. 34 (1977), 225-277.

[Me1] Z. Mebkhout, Sur le théorème de finitude de la cohomologie p-adique d'une variété affine non singulière, Amer. J. Math. 119 (1997), 1027-1081.

[Me2] Z. Mebkhout, Analogue p-adique du Théorème de Turrittin et le Théorème de la monodromie p-adique, Invent. Math. 148 (2002), 319-351.

[Mn] A.F. Monna, Analyse non-archimédienne, Springer-Verlag (New York), 1970. 
[Mo] P. Monsky, One dimensional formal cohomology, Actes du Congrès International des Mathématiciens (Nice, 1970), Tome 1, Gauthier-Villars (Paris), 1971, pp. 451-456.

[MW] P. Monsky and G. Washnitzer, Formal cohomology: I, Ann. Math. 88 (1968), 181217.

[O] A. Ogus, The convergent topos in characteristic $p$, in The Grothendieck Festschrift Vol. III, Progress in Math. 182, Birkhäuser, 1990, 133-162.

[P] F. Pham, Singularités de systèmes différentiels de Gauss-Manin, Progress in Math. 2, Birkhäuser (Boston), 1979.

[R] M. Raynaud, Géométrie analytique rigide, Mém. Soc. Math. France 39,40, 319-327.

[Sc] P. Schneider, Nonarchimedean functional analysis, Springer-Verlag (Berlin), 2002.

[Sh] A. Shiho, Crystalline fundamental groups. II. Log convergent cohomology and rigid cohomology, J. Math. Sci. Univ. Tokyo 9 (2002), 1-163.

[T1] N. Tsuzuki, Slope filtration of quasi-unipotent overconvergent $F$-isocrystals, Ann. Inst. Fourier, Grenoble 48 (1998), 379-412.

[T2] N. Tsuzuki, Finite local monodromy of overconvergent unit-root $F$-isocrystals on a curve, Amer. J. Math. 120 (1998), 1165-1190.

[T3] N. Tsuzuki, On the Gysin isomorphism of rigid cohomology, Hiroshima Math. J. 29 (1999), 479-527.

[T4] N. Tsuzuki, Morphisms of $F$-isocrystals and the finite monodromy theorem for unitroot $F$-isocrystals, Duke Math. J. 111 (2002), 385-418.

[T5] N. Tsuzuki, Cohomological descent of rigid cohomology for proper coverings, Invent. Math. 151 (2003), 101-133.

[T6] N. Tsuzuki, On base change theorem and coherence in rigid cohomology, Doc. Math. Extra Volume: Kazuya Kato's fiftieth birthday (2003), 891-918.

[vdP] M. van der Put, The cohomology of Monsky and Washnitzer, in Introductions aux cohomologies p-adiques (Luminy, 1984), Mém. Soc. Math. France 23 (1986), 33-60. 